\title{
THE EFFECT OF UNSTEADY STRETCH ON LAMINAR PREMIXED CURVED FLAMES
}

by

\author{
Meysam Sahafzadeh
}

MSc, Chemical Engineering, University of Tehran, Iran, 2012

BSc, Chemical Engineering, Arak University, Iran, 2009

\author{
A dissertation \\ presented to Ryerson University \\ in partial fulfillment of the \\ requirements for the degree of \\ Doctor of Philosophy \\ in the program of \\ Mechanical and Industrial Engineering
}

Toronto, Ontario, Canada, 2018

(C) Meysam Sahafzadeh, 2018 


\section{AUTHOR'S DECLARATION}

I hereby declare that I am the sole author of this dissertation. This is a true copy of the dissertation, including any required final revisions, as accepted by my examiners.

I authorize Ryerson University to lend this dissertation to other institutions or individuals for the purpose of scholarly research.

I further authorize Ryerson University to reproduce this dissertation by photocopying or by other means, in total or in part, at the request of other institutions or individuals for the purpose of scholarly research.

I understand that my dissertation may be made electronically available to the public. 


\title{
THE EFFECT OF UNSTEADY STRETCH ON LAMINAR PREMIXED CURVED FLAMES
}

Doctor of Philosophy, 2018

Meysam Sahafzadeh

Mechanical and Industrial Engineering

Ryerson University

\begin{abstract}
Laminar flamelets are often used to model premixed turbulent combustion. The libraries of rates of conversion from chemical to thermal enthalpies used for flamelets are typically based on counter-flow, strained laminar planar flames under steady conditions. The significance of transient strain has been discussed in the literature with most assertions being that their chemical time scales are sufficiently short compared to the turbulent time scales to treat them as quasisteady. Less discussed is the unsteady motion of a curved flame front component of stretch rate. This thesis seeks further understanding of the effect of stretch rate on premixed flames by developing and validating a model for use with transient premixed laminar flame dynamics in a cylindrically-symmetric outward radial flow geometry (i.e., inwardly propagating flame). A FORTRAN code is developed and validated which models a laminar premixed flame exposed to an oscillating mass flowrate. This code solves transient equations of continuity, momentum, energy, and individual species in radial coordinates.

In this model, flame response is studied when the flow and scalar fields remain aligned (i.e., no strain). The model is applied to conditions in which the flame expands (positive stretch)


and contracts (negative stretch) radially by the addition of the externally-defined oscillating mass flow rate. The transient response of laminar premixed flames results in amplitude decrease and phase shift increase with increasing frequency.

In order to implement the transient behaviour of flamelets in turbulent modelling more efficiently, a frequency response analysis is applied as a process characterization tool to simplify the complex non-linear behaviour using flame transfer functions. It is shown that with increasing frequency of the perturbation, when equivalence ratio is kept constant, or with decreasing equivalence ratio in the same frequency, non-linear behaviour of the flame becomes prominent. Therefore, linear models can only predict the flame behaviour with accuracy below the threshold of when the fluid and chemistry time scales are the same order of magnitude. Various nonlinear models are studied in order to find the most appropriate flame transfer function for higher frequencies to extend the predictive capabilities of these models. 


\section{Acknowledgements}

I would like to thank:

$>$ Dr. Seth Dworkin and Dr. Larry Kostiuk for providing me with the opportunity of joining their research teams and for their persistent and friendly support, encouragement, patience and advice in all steps of my $\mathrm{PhD}$.

$>$ Members of my dissertation committee, Dr. Jacob Friedman, Dr. David Naylor, Dr. Paul Walsh, and Dr. Arnaud Trouvé

$>\quad$ Natural Sciences and Engineering Research Council of Canada (NSERC)

$>$ Ryerson University for the fantastic facilities and equipment provided for this research. 
This dissertation is dedicated to my beloved parents and sisters for their kindness, devotion and for their endless support, and

Hedieh without whom none of my success would be possible. 


\section{Table of Contents}

$\begin{array}{ll}\text { Declaration } & \text { ii }\end{array}$

$\begin{array}{ll}\text { Abstract } & \text { iii }\end{array}$

Acknowledgements $\quad$ v

List of tables $\quad$ ix

List of figures $\quad x$

Nomenclature $\quad$ xii

$\begin{array}{ll}\text { Chapter 1: Introduction } & 1\end{array}$

1.1 Background and Motivation 1

1.2 Literature Review $\quad 2$

1.2.1 Numerical Simulation of Laminar Premixed Flames 3

1.2.2 The Effect of Stretch Rate on Flame Dynamics $\quad 6$

1.2.2.1 The Effect of Flow Oscillation on Turbulent Combustion 11

1.2.3 Frequency Response Analysis 13

$\begin{array}{ll}1.3 \text { Objectives } & 14\end{array}$

Chapter 2: A Numerical Algorithm for Modeling Laminar Premixed Flames Exposed to an Oscillating Flow Field in Cylindrically-Symmetric Geometry 16

2.1 Introduction 16

2.2 Model Description $\quad 17$

2.2.1 Governing Equations $\quad 20$

2.2.2 Diffusion Model 25

2.2.2.1 The Mixture-Averaged Diffusion Approximation 25

2.2.3 Mass Conservation Constraints $\quad 25$

2.2.4 Thermodynamic Properties and Transport Coefficients 27

$\begin{array}{ll}\text { 2.2.5 Chemistry Models } & 28\end{array}$

2.3 Numerical Model 31

2.3.1 Discretization Techniques $\quad 32$

2.3.2 Solution Algorithm $\quad 39$

2.3.2.1 Modified Newton's Method $\quad 39$

2.3.2.2 Linear System Solution $\quad 41$

2.4 Computational Results and Performance $\quad 42$

2.4.1 Ignition $\quad 42$

2.4.2 Steady State Conditions $\quad 47$

2.4.3 Transient Boundary Condition $\quad 47$

$\begin{array}{ll}2.5 \text { Summary } & 47\end{array}$ 
Chapter 3: A Transient Response of a Laminar Premixed Flame to a Radially

Diverging/Converging Flow

3.1 Introduction $\quad 49$

3.2 Flame Geometry and Numerical Model Specification $\quad 50$

3.2.1 Numerical Simulation $\quad 51$

3.2.2 Solution Methodology

3.3 Results and Discussion $\quad 54$

3.3.1 Steady State Condition $\quad 54$

3.3.2 Transient Periodic Flow $\quad 59$

3.3.3 Time Scale Analysis of Flame Response $\quad 70$

$\begin{array}{ll}\text { 3.4 Summary } & 77\end{array}$

Chapter 4: Using Frequency Response Analysis to Predict Unsteady Burning Rate from Unsteady Stretch Rate 80

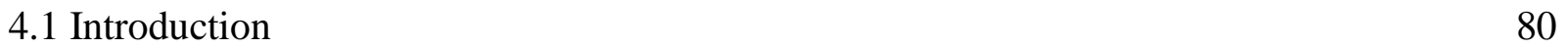

4.2 Frequency Response Analysis $\quad 81$

4.2.1 Linear First Order System Analysis $\quad 87$

4.2.2 Linear Higher Order System Analysis $\quad 91$

4.2.3 Nonlinear System Analysis 96

4.3 Prediction of a Random Input Response Using Transfer Functions 102

$\begin{array}{ll}4.4 \text { Conclusions } & 108\end{array}$

Chapter 5: Summary and Conclusions 110

$\begin{array}{ll}5.1 \text { Summary } & 110\end{array}$

$\begin{array}{ll}5.2 \text { Conclusions } & 112\end{array}$

$\begin{array}{ll}5.3 \text { Contributions } & 113\end{array}$

5.4 Recommendations for Future work 115

$\begin{array}{ll}\text { References } & 119\end{array}$ 


\section{List of tables}

Table 3. 1: Comparison of the equilibrium state and burned gas of major species and some minor

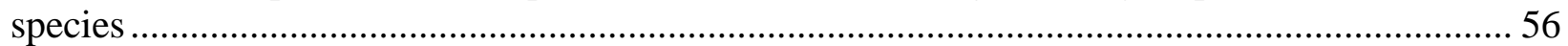

Table 3. 2: The flame speed and steady state $E I^{\prime}$ at the mean mass flow rate......................... 61

Table 3. 3: Comparison of the normalized energy index $E I$ of expanding and contracting flames at

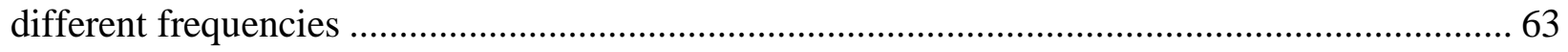

Table 4.1 Comparison of linear model prediction accuracy and deviation from linear first order

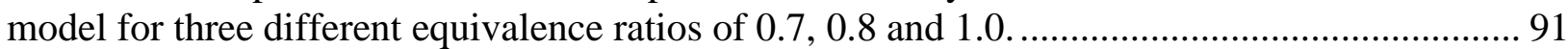




\section{List of figures}

Fig. 2.1 (a) The cylindrically-symmetric geometry (side view), (b) Stabilized stationary flame

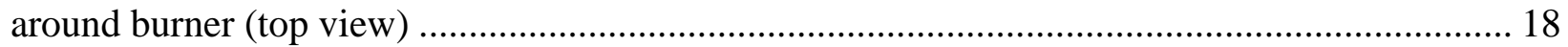

Fig. 2.2 The structure of the code including the Transport and Gas Phase Chemistry packages and the Flame Code 20

Fig. 2.3. Computational domain starting from the burner surface (inner cylinder, $r=0$ ) to outer boundary (outer cylinder $r=R$ ) ........................................................................ 36

Fig. 2.4 Evolution of temperature field and reactants mass fractions from ignition to steady state

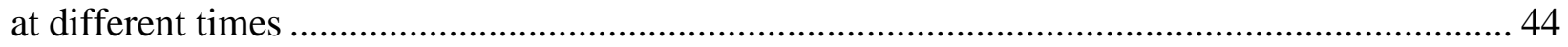

Fig. 2.5 Evolution of products mass fractions from ignition to steady state condition at different

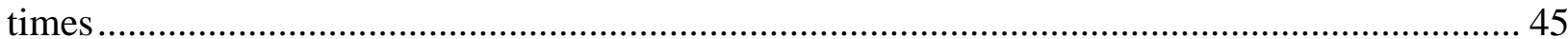

Fig. 2.6 Evolution of minor species mass fractions from ignition to steady state condition in 6 different times 46

Fig. 3.1 (a) Temperature and mass fractions of major species, (b) and (c) Species mass fractions

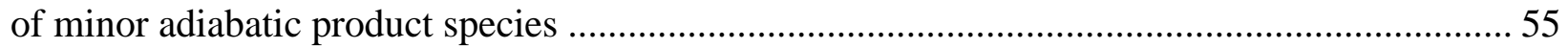
Fig. 3.2 The velocity profile of a stationary flame for comparison with flame speeds for the planar

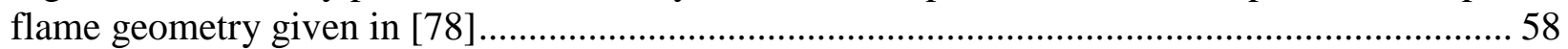
Fig. 3.3 Normalized correlation function $(C F j)$ versus cycle number $(j)$ for stoichiometric condition 62

Fig. 3.4 a) Periodic change in $E I^{\prime}$ in one cycle for quasi-steady and transient response to frequencies from $20 \mathrm{~Hz}$ to $2000 \mathrm{~Hz}$, b) $E I^{\prime}$ versus mass flow rate for quasi-steady and transient

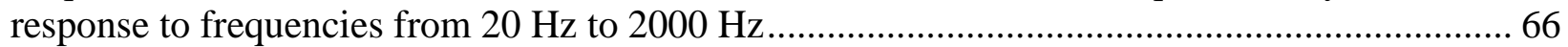
Fig. 3.5 a) The difference between maximum and minimum values of $E I^{\prime}$ ( $E I^{\prime}$ amplitude) vs. frequency for stoichiometric and fuel lean flames, b) Difference between $E I^{\prime}$ values of expanding and contracting flame at the mean mass flow rate vs. frequency for stoichiometric and fuel lean flames 68

Fig. 3.6 The effect of equivalence ratio on transient response of laminar premixed flame to various frequencies. Normalized $E I^{\prime}$ (by $E I^{\prime}$ of steady state flames) vs. normalized mass flow rate at (a) $100 \mathrm{~Hz}$, (b) $200 \mathrm{~Hz}$, (c) $500 \mathrm{~Hz}$, (d) $1000 \mathrm{~Hz}$, and (e) $2000 \mathrm{~Hz}$ for stoichiometric $(\varphi=1.0)$ and two fuel lean $(\varphi=0.8$ and $\varphi=0.7)$ flames. 69 Fig. 3.7 Changes in the radius of the isotherm (top) and stretch rate (bottom) in a cycle for

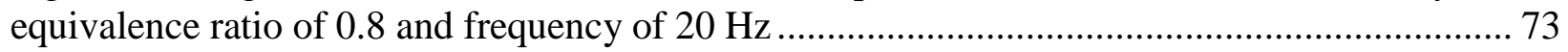
Fig. 3.8 The changes of stretch rate amplitudes vs. frequency for three different equivalence ratios

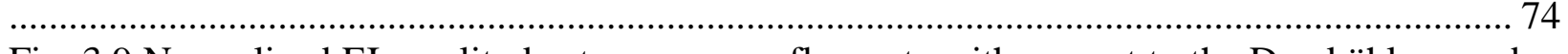
Fig. 3.9 Normalized EI amplitude at mean mass flow rate with respect to the Damköhler number calculated based on Eq. (3.8) (solid line) and Damköhler number calculated based on Eq. (3.9) (dashed line) for equivalence ratio of 0.8 . . .

Fig. 3.10 Normalized EI amplitude with respect to Damköhler number ( $D a 1)$ for three equivalence

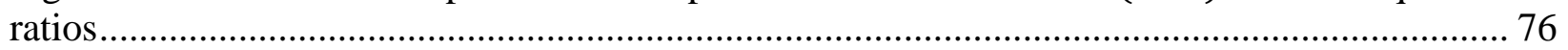
Fig. 3.11 Normalized EI amplitude at mean mass flow rate vs. Damköhler number for three equivalence ratios. 77

Fig. 4.1 Quasi steady and transient responses of flames with equivalence ratios of $\varphi=1.0, \varphi=$ 0.8 and $\varphi=0.7$ at a range of frequencies from $80 \mathrm{~Hz}$ to $2000 \mathrm{~Hz}$. 84 
Fig. 4.2 Quasi-steady and transient responses of flames with two mean flame curvatures; left side: normalized $E I^{\prime}$ in one cycle, right side: normalized $E I^{\prime}$ vs. normalized mass flow rate (equivalence ratio $\varphi=0.8$ and frequency $1000 \mathrm{~Hz}$ ). 85

Fig. 4.3. Local flame speed vs. stretch rate for equivalence ratio of 0.8 and frequencies between $20 \mathrm{~Hz}$ and $2000 \mathrm{~Hz}$ 87

Fig. 4.4 Linear first order approximation for predicting transient response, (a) for the whole range of frequencies $(\varphi=1.0)$, (b) for the low range of frequencies $(\varphi=1.0)$, and (c) deviation from linear first order behavior $(\varphi=1.0)$. (d) The whole range of frequencies $(\varphi=0.7)$, (e) for the low range of frequencies $(\varphi=0.7)$, and (f) deviation from linear first order behavior $(\varphi=0.7)$. Dots show numerical results and solid lines indicate nonlinear curve fit. 89 Fig. 4.5 Estimation of transient response of a laminar premixed flame for $\varphi=0.8$ based on low frequency data using a higher order linear model (ARX model)..... 93 Fig. 4.6 Estimation of transient response of a laminar premixed flame for $\varphi=0.8$ based on high frequency data using higher order linear model (ARX model) .... 94 Fig. 4.7 Estimating of transient response of a laminar premixed flame for $\varphi=0.8$ in low frequency zone using a nonlinear model (NARX) 101

Fig. 4.8 Estimating of transient response of a laminar premixed flame for $\varphi=0.8$ in high frequency zone using a nonlinear model (NARX) 101 Fig. 4.9 Comparison of quasi-steady and transient response (complex chemistry) of a laminar premixed flame to a random input in low frequency range $(\varphi=0.8)$, Top figure: stretch rate perturbation as the input, Bottom figure: solid line the quasi-steady response and dashed line the transient response (complex chemistry). 103 Fig. 4.10 Comparison of transient response of a laminar premixed flame and nonlinear model output in low frequency range for $\varphi=0.8 \ldots$ 104 Fig. 4.11 Comparison of quasi-steady and transient response (complex chemistry) of a laminar premixed flame to a random input in high frequency range $(\varphi=0.8)$, Top figure: stretch rate perturbation as the input, Bottom figure: solid line the quasi-steady response and dashed line the transient response (complex chemistry). 105 Fig. 4.12 Comparison of transient response a laminar premixed flame and nonlinear model output in high frequency range for $\varphi=0.8$. 105 Fig. 4.13 Comparison of quasi-steady and transient response (complex chemistry) of a laminar premixed flame to a random input in intermediate range $(\varphi=0.8)$, Top figure: stretch rate perturbation as the input, Bottom figure: solid line the quasi-steady response and dashed line the transient response (complex chemistry). 106 Fig. 4.14 Comparison of transient response of a laminar premixed flame and nonlinear model output in intermediate range for $\varphi=0.8$ 107 


\section{Nomenclature}

\section{Lowercase Roman}

\begin{tabular}{|c|c|}
\hline$a_{1}, \ldots, a_{n a}$ & Number of past output terms used to predict the current output \\
\hline$b_{1}, \ldots, b_{n b}$ & Number of past input terms used to predict the current output \\
\hline$f$ & Frequency \\
\hline$h_{k}$ & Specific enthalpy of species $k$ \\
\hline \multicolumn{2}{|c|}{$h_{n}\left(\tau_{1}, \ldots, \tau_{n}\right)$} \\
\hline$i$ & Reaction index \\
\hline$i i$ & Newton's iteration index \\
\hline$j$ & Size of array $\overrightarrow{\mathrm{X}}$ \\
\hline$j j$ & Control volume index \\
\hline$k$ & Species index \\
\hline $\boldsymbol{k}$ & gain of linear first order transfer function \\
\hline$k_{i}^{f}$ & Forward rate constant for reaction $i$ \\
\hline$k_{i}^{r}$ & Reverse rate constant for reaction $i$ \\
\hline$\dot{m}^{\prime}$ & Mass flow per unit axial length \\
\hline$\dot{m}_{0}^{\prime}$ & Mean mass flow per unit axial length \\
\hline$\langle\dot{m}\rangle$ & Normalized mass flow rate \\
\hline$n$ & Time step index \\
\hline $\boldsymbol{n}$ & Local normal to the flame front \\
\hline$n k_{i}$ & $i^{t h}$ input delay characterizes the transport delay \\
\hline$n u$ & Total number of inputs \\
\hline$p_{d}$ & Dynamic pressure \\
\hline$p_{s}$ & Static pressure \\
\hline $\mathrm{r}$ & Radius \\
\hline$r_{21}$ & Ratio of two grid sizes \\
\hline$r^{2}$ & r-square in curve fit \\
\hline
\end{tabular}


$S$

$\boldsymbol{u}$

$u(t)$

$u_{0}$

$\bar{v}^{c}$

$v_{f}$

$\dot{v}_{k i}$

$\ddot{v}_{k i}$

$\bar{v}_{k}$

$y(t)$

$z^{-1}$

Surface area of the control volume

Fluid velocity vector

ARX model input

Reference velocity

Correction velocity

Local flame speed in laboratory coordinates

Stoichiometric coefficients of reactant $k$ in forward reaction $i$

Stoichiometric coefficients of reactant $k$ in reverse reaction $i$

Diffusion velocity of species $\mathrm{k}$

ARX model output

Time-shift operator

\section{Uppercase Roman}

$A$

$\boldsymbol{A}$

$A_{i}$

$B$

$\boldsymbol{B}$

$C\left(z^{-1}\right)$

CF

$C_{p}$

$C_{p, k}$

$D a$

$D a_{1}$

$D a_{2}$

$D_{i}\left(z^{-1}\right)$

$D_{k m}$

$D_{k}$
Flame area

amplitude of linear first order transfer function intput

Experimental Arrhenius coefficient

Amplitude of oscillation

amplitude of linear first order transfer function output

Polynomial expressed in the time-shift operator

Correction factor

Specific heat at constant pressure of mixture

Specific heat at constant pressure of species $k$

Damköhler number

Damköhler number based on frequency

Damköhler number based on stretch rate

Polynomial expressed in the time-shift operator

Mixture-averaged diffusion coefficient

Diffusion coefficient of species $k$ 


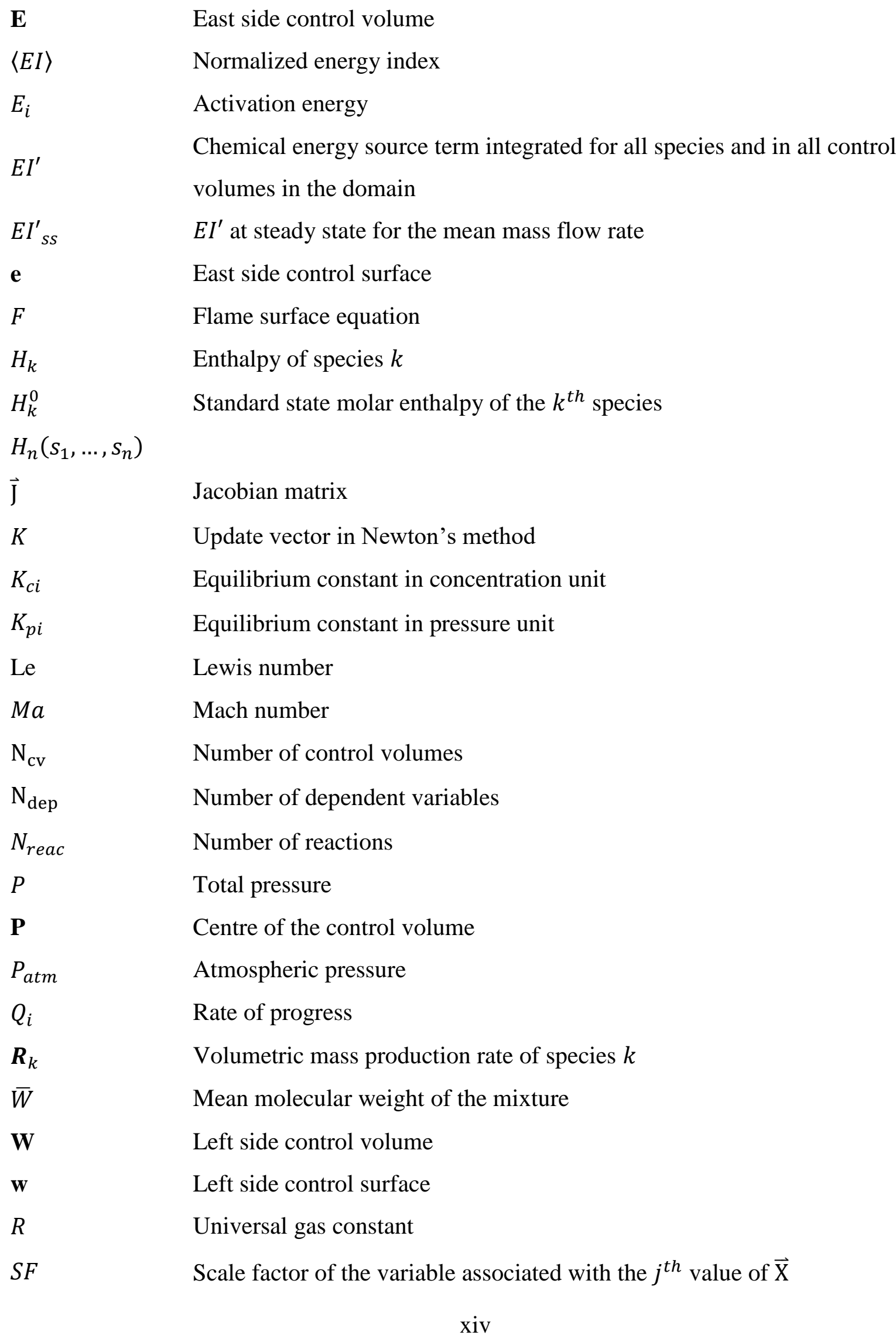




$\begin{array}{ll}S_{k}^{0} & \text { Standard state molar entropy of the } k^{t h} \text { species } \\ S_{L}^{0} & \text { Unstretched laminar flame velocity } \\ T & \text { Temperature } \\ T o l & \text { Newton's method tolerance } \\ T_{u} & \text { Temperature at the inlet boundary } \\ \boldsymbol{V} & \text { Fluid velocity } \\ \mathbb{V}^{\prime}(i) & \text { Volume of cell } i \text { (per unit axial length) } \\ W_{k} & \text { Molecular weight of species } k \\ \overrightarrow{\mathrm{X}} & \text { Array of all dependent variables } \\ \overrightarrow{\mathrm{X}}_{0} & \text { Initial guess for dependent variables } \\ Y_{k} & \text { Mass fraction of species } k \\ Y_{k, u} & \text { Mass fraction of species } k \text { at the inlet boundary }\end{array}$

\section{Lowercase Greek}

$\begin{array}{ll}\alpha & \text { Thermal diffusivity } \\ \beta_{i} & \text { Temperature exponent } \\ \rho & \text { Density } \\ \kappa & \text { Stretch rate } \\ \mu & \text { Dynamic viscosity } \\ \lambda & \text { Mixture coefficient of thermal conductivity } \\ \dot{\omega}_{k} & \text { Molar production rate of species } k \\ \omega & \text { Angular frequency } \\ \sigma & \text { Artificial damping term coefficient } \\ \delta & \text { Jacobian matrix perturbation } \\ \boldsymbol{\tau} & \text { Time constant } \\ \boldsymbol{\theta} & \text { Period of oscillation } \\ \xi & \text { Nonlinearity parameter } \\ \boldsymbol{\epsilon} & \text { Solution on the corresponding grid }\end{array}$


$\varepsilon$

$\varphi$

$\varnothing$

\section{Uppercase Greek}

$\begin{array}{ll}\overrightarrow{\mathcal{F}} & \text { Residual array in Newton's method } \\ \mathcal{M} & \text { Nonlinearity estimator } \\ \mathcal{N} & \text { Source term } \\ \mathcal{R} & \text { Set of } N_{\text {reac }} \text { reactions } \\ \Gamma & \text { Diffusion parameter } \\ \chi_{k} & \text { Chemical symbol of species } k \\ \forall & \text { Control volume }\end{array}$




\section{Chapter 1: Introduction}

\subsection{Background and Motivation}

Numerical simulation of turbulent combustion has significance in practical devices, such as industrial furnaces, stationary gas turbines, aero-combustors or internal combustion engines. The goal of these simulations is to design more efficient and cleaner combustion systems. Premixed turbulent combustion is a canonical case for the reactant state of some practical combustion systems, such as HCCI (Homogeneous Charge Compression Ignition) engines. The modelling of such systems is a challenging task due to the unsteady, multi-component and multidimensional nature, and the large range of length and time scales of these flows. These complexities have been discussed in the literature as part of the accurate determination of the rate of conversion of reactants to products, and the sensitivity of the turbulent burning rate to the geometry of the flame [1].

The range of potential couplings between the various scales of turbulence and the chemistry occurring within the structure of a flame are typically captured by ratios of time scales known as the Damkohler $(\mathrm{Da})$ and Karlovitz $(\mathrm{Ka})$ numbers. At large Damkohler numbers the small-scale chemistry is essentially decoupled from large-scale flow features. In these instances, premixed turbulent flames have often been viewed as ensembles of premixed laminar stretched flames that are wrinkled by the turbulent flow field, in the so-called 'flamelet' approach [2]. The local burning characteristics have been calculated from steady strained laminar flames as a function of equivalence ratio, pressure, temperature, and strain rate [3], but without consideration of potential impacts of spatial gradients of these quantities over the flame surface or any temporal gradient. The effect of strain rate on the laminar premixed flame has been examined by many studies (e.g., 
[4]) and it has been shown how different phenomena can affect local burning rates when small sections of the turbulent flame experience a range of stretch rates locally [5].

Premixed turbulent flames that are employed in low emission combustion systems have been the focus of recent work due to their being prone to combustion instabilities [6]. The challenges related to these flames have been discussed extensively because of their time-dependent and multidimensional aspects. One of the unresolved challenges related to the time dependent nature of turbulent flame modeling is the transient response of flamelets to unsteady stretch rates. Several efforts have been made to address the importance of unsteady strain rate on the burning rate and heat release of flamelets [7]. Less discussed is the unsteady motion of a curved flame front component of stretch rate. In flamelet modeling, the properties of a turbulent flame are calculated from an ensemble of laminar flamelets. The structure and information of these flamelets are stored in a library of strained laminar flames that contain scalar quantities as a function of equivalence ratio, pressure, temperature, and strain rate. Since these libraries are based on the steady strained flames, the unsteady stretch rate could play an important role in estimation of the actual local burning rate and heat release. Therefore, in the continuing effort to address the effect of unsteady stretch rate on the flamelet dynamics, the transient response of laminar premixed flames is studied as a potential modification to the flamelet library, by considering the effects of curved flame front motion. The method that is proposed in this thesis is to capture the transient effects of a curved flame in a mathematical relationship such as transfer functions.

\subsection{Literature Review}

In this section, a brief literature review is presented to facilitate a concise overview of the previous investigations into numerical simulation of laminar premixed flames, transient response 
of stretched laminar flames, flame transfer functions, and their applications in turbulent combustion modeling using flamelet libraries. It is intended to illustrate the areas where further research is required. More extensive literature reviews on specific topics are provided at the beginning of Chapters 2-4.

\subsubsection{Numerical Simulation of Laminar Premixed Flames}

Flame structure and combustion products (especially environmental pollutants) are two major aspects of practical combustion devices that need to be comprehensively analyzed and understood. Understanding combustion behavior in terms of these aspects and modeling these phenomena in detail can be achieved by combining combustion theory with available high performance computing technology [8]. The computational revolution in combustion and the development of reliable numerical methods for simulating flames using detailed chemical kinetic and transport models began decades ago upon the advent of digital computers. The accurate and efficient numerical solution of flames is a relatively recent development in the history of combustion science and an ongoing research area in the computational combustion field.

In this thesis, the initial objective is to develop an algorithm to simulate a time-dependent laminar premixed flame in cylindrical geometry with detailed chemistry and thermodynamic properties. A laminar flame in a one dimensional radial outward flow field not only permits the

study of the unstretched structure of a reacting flow but also enables the researchers to analyze the flame transient response when exposed to a perturbation such as a sinusoidal variation in mass flow rate. The motion of a flame front in this geometry results in a stretched flame, while the tangential strain rate is non-existent. The unique feature of this geometry is that it generates 
positive and negative stretch rates in a flame and enables researchers to analyze the flame structure and dynamics in a wide range of stretch rates without the effects of having a strain rate.

Hirschfelder et al. [9] and Spalding [10] were among the first researchers who applied numerical methods to model combustion. Numerical simulation of laminar premixed flames has essentially followed two approaches [11]. The first one is adopted from Hirschfelder et al. [9], who introduced a solution to the time-independent one-dimensional equation in a two-point boundary value problem. In the second approach, Spalding [10] described the full time-dependent equations not requiring that the flame speed be known a priori. This description appears to be mathematically more straight-forward than the steady-state approach. Spalding [10] solved a steady adiabatic chemical kinetics problem which included a three-step mechanism. The approach Spalding took was marching the time-dependent system of nonlinear partial differential equations in time to reach the steady-state solution.

In the next major advancement in combustion modeling, Dixon-Lewis [12] used timedependent partial differential equation solution methods to study hydrogen/oxygen flames. Since then, modeling one-dimensional and two-dimensional laminar flames has become the interest of several researchers in this field $[13,14,15,16]$. A promising approach was to use finite-difference or finite-volume discretizations to linearize the set of partial differential equations. The equations are then solved using a damped, modified Newton's method. Early works using Newton's method have included simulation of burner-stabilized methane-air premixed flames [17] and counter-flow methane-air diffusion flames [18].

Turbulent combustion modeling has become the focus of several research groups since the power of computers increased in the 1990s. Methods of simulating turbulent flames that are commonly found in the literature include unsteady flamelet modeling [19], PDF modeling [20, 
21], Direct Numerical Solution (DNS) [22], and large-eddy simulation [23]. With regard to direct bearing on algorithmic issues for the aforementioned turbulent flame simulation approaches, the accurate and efficient computation of time-dependent laminar flames needs to be modified and improved in terms of accuracy and complexity. For example, DNS of turbulent reacting flows has been the topic of ongoing research projects recently due to its capability to capture all the time scales and therefore the transient effects in the simulations. However, with regard to the current computational resources, this method is not efficient in combustion systems with complex geometries.

In computational combustion, there are four governing equations; continuity, momentum, energy, and mass species, that need to be solved. There are different ways to formulate the fluid dynamic problems, while the mass fractions and the temperature are typically obtained by solving the species and energy equations. The first method is called the "primitive variable formulation" in which the mathematical model of the transient laminar flame becomes a parabolic set of governing equations. The conservation equations in terms of primitive variables is illustrated in the next chapter of this thesis (chapter 2). In primitive variable models, as applied in this work, the unknowns include pressure, radial velocities, temperature, and species mass fractions. This method has been implemented successfully in different works such as [24, 25]. In this formulation, while the velocity profile is obtained by the momentum equation, the pressure field can be dealt with in different ways. The first method, which will be applied in this thesis, is solving the continuity equation together with the momentum and other governing equations in a fully coupled approach. The other way of recovering pressure is estimation in one step of a segregated solution algorithm by solving a Poisson equation for the pressure, obtained by taking the divergence of the momentum equations [26]. As intrinsically there are two governing equations of continuity and momentum, 
the discretization of the pressure profile should be consistent with the discretization of the continuity equation which can be obtained on a staggered grid. It should be noted that staggered mesh schemes have drawbacks in complex geometries, in non-orthogonal curvilinear coordinates, and when using sophisticated numerical techniques such as locally adapted grids or multi-grid methods [26] which are not relevant in this work.

\subsubsection{The Effect of Stretch Rate on Flame Dynamics}

Stretch rate $(\kappa)$ as a mechanism to affect the rate of combustion was first introduced by Karlovitz et al. [27] as the Lagrangian time $(t)$ derivative of an element of the flame surface area (A) as in Eq. (1.1).

$$
\kappa=(1 / A)(d A / d t)
$$

In that work, a planar combustion wave, exposed to a velocity gradient, was analyzed and the effect of curvature was ignored. Markstein [28] discussed the impact of the motion of curved flame fronts normal to itself for the first time. The combination of these two effects; namely, the underlying hydrodynamic strain and the flame surface curvature effects on an interface have been expressed as stretch rate [29, 30]. Matalon [29] introduced an expression (Eq. (1.2)) for calculating stretch rate of a flame in an arbitrary shape on the flame surface indicated by the function $F(X, t)=$ 0.

$$
\kappa=\left\{v_{f} \nabla \cdot \boldsymbol{n}-\boldsymbol{n} \cdot \nabla \times(\boldsymbol{V} \times \boldsymbol{n})\right\}_{F=0}
$$


where $\boldsymbol{V}$ is the fluid velocity, $\boldsymbol{n}$ is the local normal to the flame front and parallel to the gradient in the scalar field (local normal is considered positive when pointing from reactants to products), $v_{f}$ is the speed of an identifiable flame surface feature and thus can be the local flame speed in laboratory coordinates. The first term on the right side of Eq. (1.2) represents the flame curvature effects and results from the divergent/convergent flow field and motion of the flame in that flow field. The second term illustrates the tangential strain rate and results from a non-uniform flow field across a scalar field of the flame. The summation of these two terms creates an equivalence between the strain and curvature effects, but that is strictly true only for an interface, while flames of a finite thickness and multiple scalar species add uncertainty to this equivalence and introduce some unique challenges regarding which interface to consider [31, 32].

In counter-flow configurations, due to the planar flame shape, the divergence of the scalar field $(\nabla . \boldsymbol{n}=\mathbf{0})$ becomes zero and subsequently the first term in Eq. (1.2) is eliminated; however, misalignment of flow and scalar fields $((\boldsymbol{V} \times \boldsymbol{n}) \neq 0)$ results in a non-zero value for the second term. Therefore, the laminar premixed flame in this geometry is strained. It should be noted that in Eq. (1.2), a fixed frame of reference was used in developing the equation. In this condition, the effect of curvature for a stationary flame appears implicitly in the second term of Eq. (1.2). Thus, when the second term is expanded using appropriate vector identities, it results in $[-(V . n) \nabla . n]$. The other terms resulting from the expansion of the second term are not proportional to curvature. Therefore, the total contribution of the flame curvature to stretch rate can be shown using Eq. (1.3):

$$
\kappa=-S_{f} \nabla \cdot n=-\left(V \cdot n-v_{n}\right) \nabla \cdot n
$$


For instance, in an expanding (propagating) cylindrical or spherical flame, due to the radial uniform flow through the flame, stretch is generated only due to flame motion. In this condition, $S_{f}=v_{n}$ and $V=0$, which means the flame displacement speed is equal to the flame burning rate. Therefore, the stretch is calculated based on the radius of the propagating flame front as follows.

$$
V \cdot n=0, S_{f}=v_{n}=\frac{d R}{d t}, \kappa=v_{n} \nabla \cdot n=\frac{1}{R} \frac{d R}{d t}
$$

Another representation of total stretch [33] is when the frame of reference is attached to the fluid parcel (Eq. (1.5)). In this condition, the flame is considered to be both strained and curved.

$$
\kappa=(\boldsymbol{I}-n n): \nabla u+\left(v_{n}\right) \nabla \cdot n
$$

The first term is attributed to hydrodynamic (tangential) strain caused by the flow and the second term is attributed to the stretch experienced by a curved surface moving with $v_{n}$ (laminar flame speed). Equation (1.5) can be rewritten as follows:

$$
\kappa=(\boldsymbol{I}-n n): \nabla u_{t}+\left(u \cdot n+v_{n}\right) \nabla \cdot n
$$

where $u . n$ is the flow velocity in the normal direction. The first term on the right side denotes the total tangential strain and the second term denotes the total normal strain. Therefore, at steady state, the balance of normal strain and the curvature $\left(u . n=-v_{n}\right)$ results in zero stretch rate. Equation (1.6) is equivalent to Eq. (1.2). With regard to the flame geometry in this study, the first approach was taken to calculate the total stretch rate. The disadvantage of using the second 
approach is to calculate the normal strain and local flame burning rate by selecting an arbitrary iso-surface. In the current research, due to the uniform radial flow through the flame front, it is more convenient to use the radial position of the flame iso-surface to calculate the total stretch rate. However, in flames with a misalignment between flow field and flame front in their structures, the second approach provides more convenience due to the total strain rate term.

A time-dependent investigation in this geometry was performed by Saitoh and Otsuka [34] for both premixed and diffusion flames numerically and experimentally. They varied the velocity normal to the stagnation plane sinusoidally around its mean. They concluded that the positional amplitude of temperature and concentration fluctuations decreased with increasing oscillation frequency. In a similar work, Stahl and Warnatz [3] carried out a numerical investigation on the transient response of strained flamelets. They studied the influence of time-dependent sinusoidal change of strain rate on the flame front behaviour. The dependence of flame oscillation amplitude and phase shift with frequency of the strain rate was analyzed and it was concluded that the amplitude of flame position oscillation decreases and the phase shift increases with an increase in frequency.

These studies showed that in a counter-flow configuration, the response of a strained flame to a periodic change in flow velocity and strain rate is not instantaneous, implying limitations in validity of the quasi-steady laminar flamelet assumption in premixed turbulent modeling. As part of this endeavour, Petrov and Ghoniem [35] revisited the validity of this assumption by analyzing the transient response of premixed methane-air laminar flames to both stepwise and periodic changes in strain rate over a range of Lewis numbers and flame temperatures. They concluded in a flamelet model, the response of laminar premixed flames can be considered instantaneous for two conditions. First, over the entire range of Lewis number only for high flame temperatures, and 
second, intermediate flame temperature when Lewis number equals unity. Thus, for low and intermediate flame temperatures and non-unity Lewis numbers, the model could be modified to reflect the lag between flow and flame.

In order to stress the significance of unsteady stretch rate, dynamics of a laminar stretched hydrogen flame have been analyzed when the flame front was strained and curved [36]. Lauvergne and Egolfopoulos [37] studied the transient response of laminar premixed flames in a counter-flow geometry wherein the flame is strained and was exposed to sinusoidal perturbations of fuel concentration as a boundary condition. Although the effects of curvature were not considered, it showed many valuable insights including the quasi-steady response at low frequencies (lower than a cut-off frequency) and a significantly attenuated response with a phase lag at high frequencies. In their study, due to the fact that chemical time scales are shorter than flow time scales, no accumulation of mass was observed, which is different from when the flame is exposed to velocity or stretch rate change. Huang et al. [38] theoretically studied the response of a premixed flame in the counter-flow geometry with a fluctuating strain rate. The range of oscillation frequency was below $100 \mathrm{~Hz}$ and the strain rate amplitude range was from $10 \%$ to $40 \%$ of the mean value. Important results were obtained from this study; first, strained flames do not respond instantaneously to temporal variations in the flow field, as shown in [34] and [7]; second, flamelet models that use a quasi-steady assumption in predicting turbulent flame properties had to be modified to include unsteady effects. For small amplitudes of perturbation, the flame displacement response was linear. However, by increasing the amplitudes of perturbations, nonlinear effects dominate the flame behaviour such that the response remains periodic, but the output could not be expressed as one sinusoidal function. The phase lag in the flame's response was seen to increase monotonically with frequency and approached $90^{\circ}$ at very high frequencies. 
The above investigations were concerned with the transient response of strained flames, while fewer studies have been concerned with the effects of curvature. Giannakopoulos et al. [39] numerically studied the effect of flame curvature by using spherically outwardly propagating flames. This geometry has also been used extensively to study the influence of flow rate on stabilized [40] and flame-stretch interactions in premixed flames (e.g. [41]). In this geometry, due to the alignment of flow and scalar fields, the tangential strain rate (the second term in Eq. (1.2)) is null, but the flame is stretched because of the motion of the curved flame. In this condition, flame stretch varies with time as the flame propagates outward and has a positive value at all times. Therefore, in the present study, the transient response of a laminar flame in a cylindrical geometry (one-dimensional outward radial flow) could unravel unique aspects of reacting flows dynamics by considering both positive and negative stretch rate.

\subsubsection{The Effect of Flow Oscillation on Turbulent Combustion}

As mentioned before, the dynamics of laminar flamelets are of importance in defining turbulent combustion modeling using the flamelet libraries approach. In one of the first studies on the influence of large scale wrinkling on turbulent burning velocity, Berestycki and Sivashinsky [42] showed that with an increase in amplitude of flow fluctuations the average burning rate increases. The importance of the aforementioned study was in the relationship between oscillation of burning velocity and local flame front curvature. Aldredge [43, 44], considered a more general flow field under the condition of weak flame stretch to analyze the dependency of turbulent premixed burning velocity on flow intensity, which agreed with the results in [42]. It was concluded that at least a part of the "bending effect," which is normally seen in combustion occurring in high-intensity turbulence [45], results from the weak local flame stretch that modifies 
the local normal flame speed. In premixed turbulent combustion, burning velocity is enhanced linearly by low-intensity turbulence. For a better understanding of the relationship between flame surface advection and the intensity of the excitation flow, Aldredge [46] studied the advection of a flame front in a transient periodic flow (spatially and temporally) wherein different levels of turbulent intensity (low, moderate and large) of flow perturbations were considered analytically and numerically. The results were consistent with those obtained in the earlier investigations [44]. In recent research, Aldredge [47] studied the flame surface and burning rate increase due to wrinkling, by analyzing the effect of multiscale periodic transient flow on isothermal-flame propagation. It has been concluded that flame behaves differently in low, intermediate and high intensities. In Aldredge's work, due to the restriction of transient flame surface growth, the local normal propagation speed is kept constant and equal to the adiabatic planar flame (laminar flame speed). However, in the present thesis, the periodic transient flow is applied to a curved flame front, which experiences no strain. All of these studies have shown the important effects of turbulent intensity on local burning velocity and from a flamelet modeling point of view, they confirm that the unsteady stretch rate effects and transient response of laminar flames should be considered in flamelet libraries. However, none of the above studies indicated the individual effect of motion of a curved flame on local burning velocity and flamelet dynamics. Therefore, in the current thesis, after analyzing the significance of time dependent motion of laminar flame fronts on flame behaviour as a potential modification to the flamelet libraries, frequency response analysis is presented as a mathematical tool to capture the flame response. 


\subsubsection{Frequency Response Analysis}

With regard to the fact that defining a comprehensive library based on unsteady flamelet information is not efficient, it is more rational to link the transient response of laminar flames to existing steady flamelet libraries. One of the applicable and efficient techniques that can be applied is capturing transient effects of laminar premixed flames in a simple mathematical relation such as transfer functions. Frequency response analysis is often used in order to generate these functions.

Laminar premixed flames are described by a system of partial differential equations and algebraic constraints that represent the conservation of total mass, momentum, energy, and the evolution of individual species mass under the mechanisms of convection, diffusion, and chemical reaction. The equations are highly nonlinear and strongly coupled across length and time scales. It should be noted that in cases of using reduced chemistry and simple transport properties, the transient response of a stretched flame cannot be captured accurately due to the change in the flame structure [48]. Therefore, solving a set of governing equations to include the effects of transient response of laminar flames in forming flamelet libraries is time consuming, inefficient, and often impractical in many turbulent applications. It has been suggested that in order to implement the effect of transient response of the stretched flames in turbulent combustion modeling, the overall Markstein number could be modified with the frequency and flame transit time [36]. Another approach to this problem for a more practical application is to represent the frequency response analysis of flames in the form of flame transfer functions. This concept has been used in combustion [49] in order to study flame instabilities. Linear [50] and non-linear [51] frequency response analyses of laminar [52] and turbulent [53] premixed flames have been considered. The motivation is to describe the flame response to flow velocity perturbations as a flame transfer 
function in order to study and model flame instabilities due to acoustic waves. In some studies, such as [54], the transfer function is defined by the ratio of the normalized flame area to velocity fluctuations.

The theoretical studies in [55] used a $G$-equation and characterized the flame front by its mean and perturbation components. Then, solving by Laplace transforms, the frequency response can be obtained. However, in this study, the frequency response analysis was obtained based on several numerical simulations of a laminar premixed flame.

Thermo-acoustic instabilities have been studied using a nonlinear describing function (instead of a linear flame transfer function), which was determined experimentally [51]. The flame response was estimated as a function of frequency and amplitude of perturbation acting on the combustion region. It was concluded that nonlinear mechanisms dominate the dynamics of real systems and give a better understanding of the flame dynamics.

In order to test the capability of the transfer functions, some random-looking input perturbations that include discrete low, intermediate, and high frequency oscillations are applied to the system and are compared to the numerical simulation with complex chemistry results. Low and high frequency perturbations show excellent accuracy. Thus, transfer functions show a promising methodology to predict the flamelet response to a change in upstream flow field.

\subsection{Objectives}

The main objective of this thesis is to investigate the effects of the motion of a curved flame (unsteady stretch rate due to flame curvature) on flame dynamics and its importance in modifying steady flamelet libraries in order to model turbulent combustion. The following secondary objectives will serve as milestones to achieve this main objective: 
$>$ Developing and validating a transient code to simulate a laminar flame in cylindrically-symmetric radial flow geometry (Chapter 2).

$>$ Proposing an approach to revisit the steady state approach in laminar flamelet models by analyzing transient response of laminar premixed flames when exposed to an oscillating flow field (Chapter 3).

Generating transfer functions (flame describing functions) based on transient response datasets in order to predict the flame behavior and capture the flame response when exposed to a change in upstream flow field. This comprises studying first order linear, higher order linear, and nonlinear systems to propose the best accuracy (Chapter 4). 


\section{Chapter 2: A Numerical Algorithm for Modeling Laminar Premixed Flames Exposed to an Oscillating Flow Field in Cylindrically-Symmetric Geometry}

This chapter is based on the following paper:

Meysam Sahafzadeh, Larry W. Kostiuk, Seth B. Dworkin, “A Numerical Algorithm for Modeling Laminar Premixed Flames Exposed to an Oscillating Flow Field in Cylindrically-Symmetric Geometry," Submitted to Computer Physics Communications (under review), October 2017.

\subsection{Introduction}

Adding more accuracy to simulation algorithms and making them more applicable is an ongoing goal of combustion modeling. One of the most significant opportunities to improve these algorithms is adding time dependence to detailed laminar flame simulations with detailed chemistry models, so the structure of the flame can be analyzed when it is subjected to a transient process, such as ignition or fluctuation in inlet mass flow rate of reactants $[56,14]$. It is vital to include major and minor species in addition to detailed chemistry in order to capture all thermochemical and hydrodynamic aspects of the flame structure. These algorithms have been developed to simulate flames in various geometries such as one- or multi-dimensional planar flames which are stabilized in the counter-flow domain or propagating spherical geometry. However, flames in all of these shapes experience a positive stretch rate, which results from either strain in the counter-flow planar geometry or propagating spherical flame front (which can be observed in zero gravity combustion experiments). It is worth mentioning that positive stretch rate is a fluid element defined by its normal pointing from reactants to products will always be increasing its area in the perpendicular direction as it passes through the flame. In this regard, this thesis initially studies the development of such a mathematical model and numerical methods for 
unsteady laminar premixed flame problems. The particular emphasis of this model is on computational algorithms that enable their fully coupled solution, including all species with their transport and thermodynamics properties and detailed chemistry. With this algorithm, one can estimate the flame structure and trace the flame dynamics in fast transient conditions. Another novelty of the presented algorithm is the modeling of the laminar flame in cylindrical coordinates, which allows the study of unstretched, positively stretched, and negatively stretched properties of a laminar premixed flame in one geometry. All of these forms of stretch are observed in a turbulent premixed flame [5], thereby providing relevance to high Damköhler Number flame that occur in practical combustion devices. In the following chapter of this thesis, the governing equations, boundary conditions, transport fluxes, thermodynamic properties and kinetic modeling are explained in detail. Then, the numerical technique and solution methods are presented. In the end

of this chapter, some of the main applications of the algorithm including flame structure and burning rate calculation in steady-state conditions, and transient responses to sinusoidal variations in inlet mass flow rate, are discussed.

\subsection{Model Description}

It is desired to create one-dimensional (radially) outward flow of a pre-mixture of $\mathrm{CH}_{4}$ and air $\left(\mathrm{O}_{2}\right.$ and $\left.\mathrm{N}_{2}\right)$ of specifiable composition and temperature, in cylindrical geometry, as depicted in Fig. 2.1. The mass flow rate of reactants $(\dot{m})$ was imagined to occur along the axial coordinate (Fig. 2.1-a) and then be distributed radially outward through a porous cylinder with characteristics to create a uniform diverging flow. It is at the exit plane of the porous cylinder that the computational domain begins. The computational domain ends at the surface of a larger cylinder where the identical mass flow is extracted. The system is assumed to be of sufficient axial length 
(i.e., the height of the cylinder) and neglecting gravity, a one-dimensional flow in the radial direction is obtained, which is independent of the axial or azimuthal coordinates.

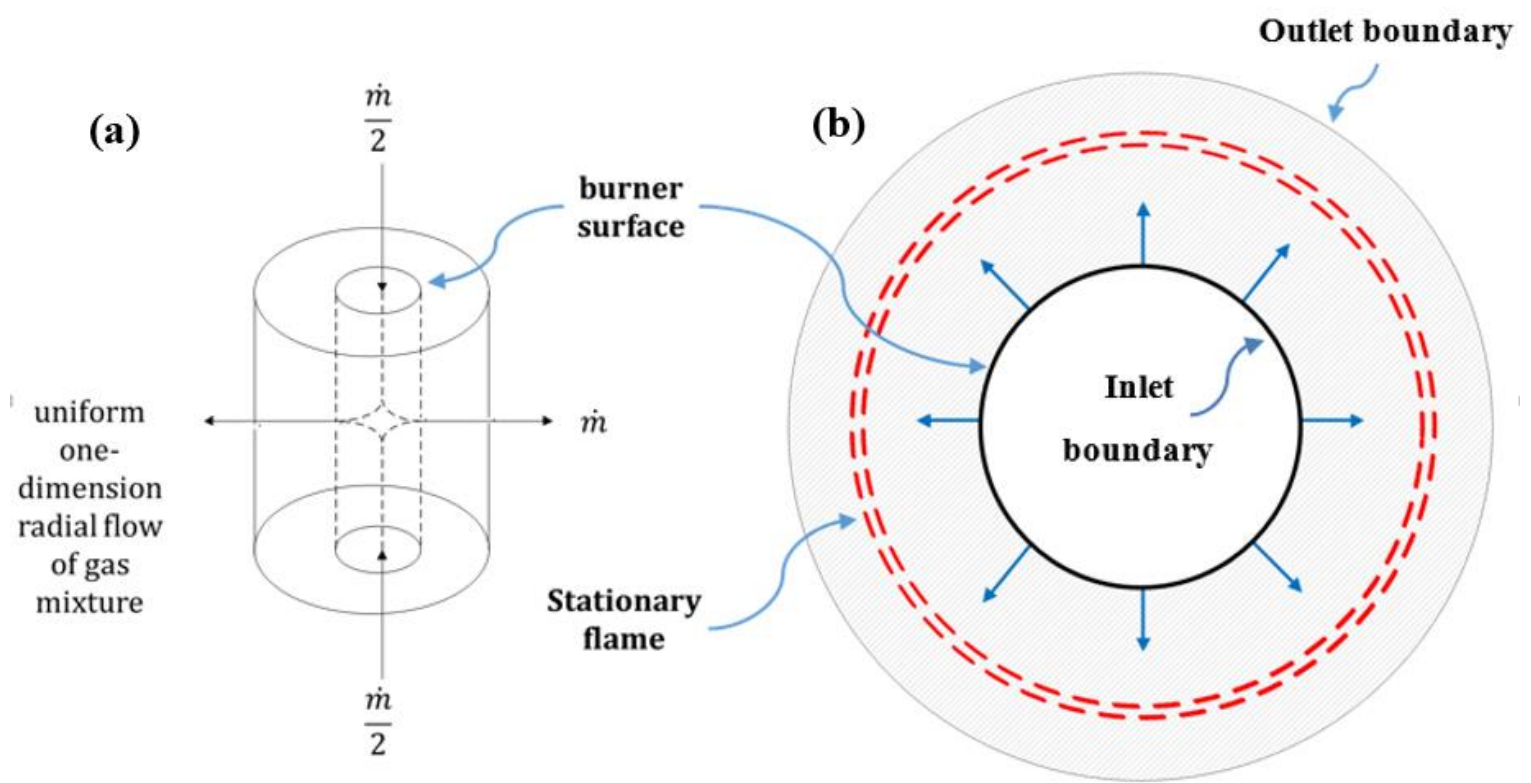

Fig. 2.1 (a) The cylindrically-symmetric geometry (side view), (b) Stabilized stationary flame around burner (top view)

When the computational domain (grey area in Fig. 2.1-b) is initially filled with pure nitrogen at high temperature (e.g., $1800 \mathrm{~K})$, the incoming reactant gas mixture is ignited and a cylindrical flame (dotted lines in Fig. 2.1-b) forms between the inlet and outlet boundaries. The result is an inwardly propagating flame, and this flame will reach steady state (i.e., becoming stationary in space) if there is a convection velocity within the computational domain that balances the diffusive transport of heat and mass associated with the flame. Although this flame has not been studied experimentally due to difficulties in buoyant stability, it represents unique features for theoretical and numerical studies of laminar premixed flames for two main reasons. First, similar to a stationary spherical flame [57], the flow field in the domain is radially diverging. In 
such a condition, the strain of the flow is balanced by the curvature of the flame, and thus the total stretch rate equals zero, which is essential to analyze the separate effects of curvature and strain rate. Second, oscillating the flow at the inlet boundary enables the study of flame creation (expanding) and destruction (contracting).

The structure of the code is illustrated in Fig. 2.2 for a better understanding of the procedure taken in laminar flame computation. The code includes three main sections; namely, transport, gas phase chemistry, and the flame solver. As will be explained in detail in the following sections, 'transport' links to a properties' library required to estimate transport physics, while 'gas phase chemistry' includes all elements, species, reactions, and thermodynamic properties. The centre of the code is called "flame solver" which includes all governing equations, boundary conditions and the solution method. The output of the flame code is written to solution DAT files, which can be modified to plot the results at specifiable time steps. In the default mode of the code, the solutions are written every 100 time steps. 
Transport

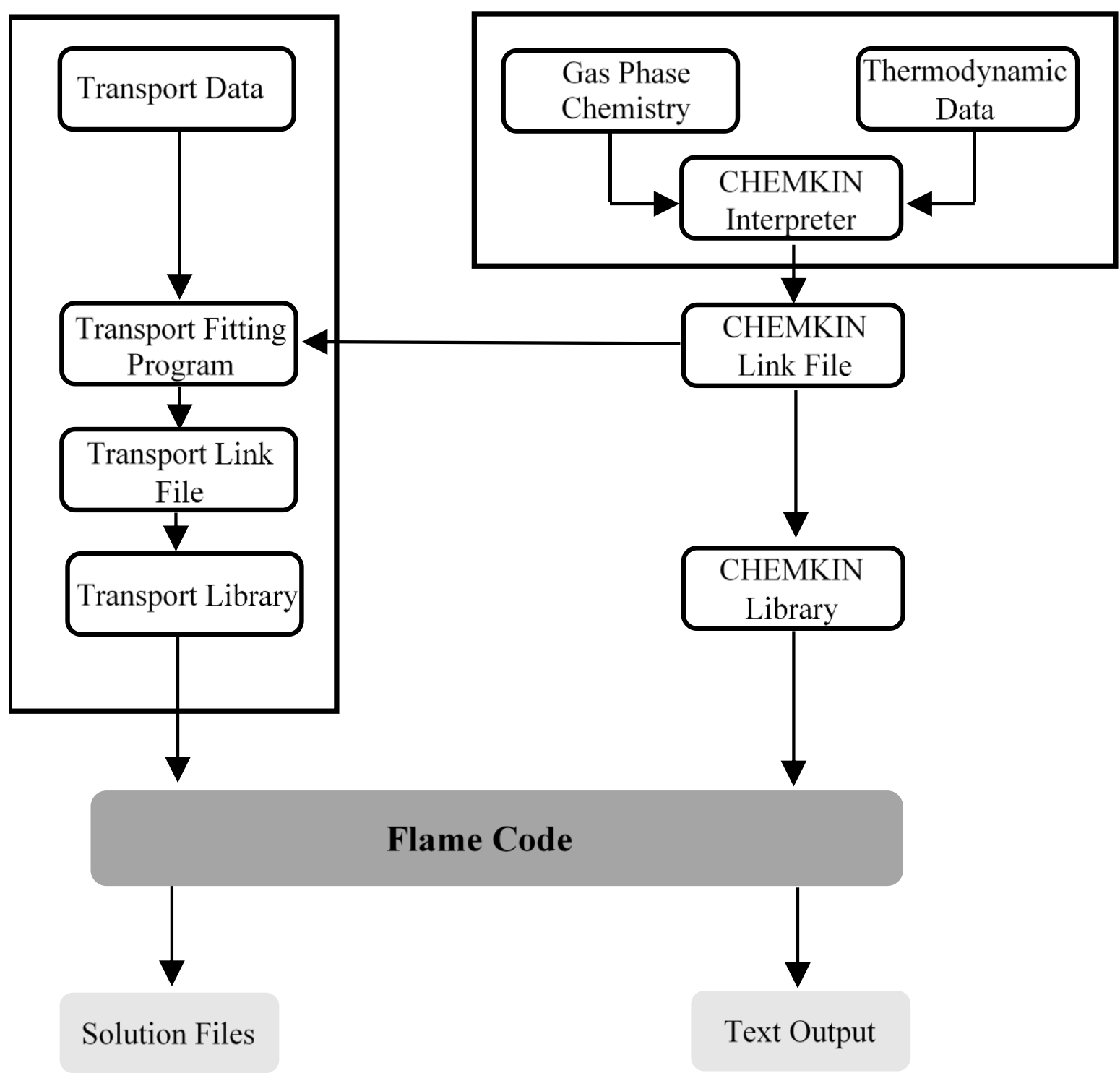

Fig. 2.2 The structure of the code including the Transport and Gas Phase Chemistry packages and the Flame Code

\subsubsection{Governing Equations}

Laminar premixed flames are governed by a complicated system of partial differential equations and algebraic constraints that describe the conservation of total mass and energy, and the balance of momentum and the creation and destruction of individual species mass under the 
mechanisms of convection, diffusion, and chemical reaction. The equations are highly nonlinear and strongly coupled across length and time scales. In addition, in a combustion system, detailed chemistry models and reactions should be employed which makes the system of equations more nonlinear and complex.

This model is usually formulated in terms of the "primitive variables" - pressure, velocity, temperature, and the species mass fractions. For this specific geometry:

Conservation of total mass:

$$
\frac{\partial \rho}{\partial t}+\frac{1}{r} \frac{\partial}{\partial r}(\rho \boldsymbol{u} r)=0
$$

where $\boldsymbol{u}$ is the fluid velocity vector, $\rho$ the density, $r$ the radius, and $t$ the time. Balance of momentum:

$\rho \frac{\partial \boldsymbol{u}}{\partial t}+\rho \boldsymbol{u} \frac{\partial \boldsymbol{u}}{\partial r}+\frac{\partial P}{\partial r}-\frac{2}{r} \frac{\partial}{\partial r}\left(r \mu \frac{\partial \boldsymbol{u}}{\partial r}\right)+\frac{2}{3} \frac{1}{r} \frac{\partial}{\partial r}\left(\mu \frac{\partial}{\partial r}(r \boldsymbol{u})\right)+\frac{2 \mu \boldsymbol{u}}{r^{2}}-\frac{2}{3} \frac{\mu}{r^{2}} \frac{\partial}{\partial r}(r \boldsymbol{u})=0$

In Eq. (2.2), $P$ is the total pressure including static and dynamic pressures; and is $\mu$, the dynamic viscosity of the mixture. As is common in the study of low speed flows of dilute gases, the bulk viscosity is assumed to be negligible [58]. According to [59], for a laminar methane air flame in the range of equivalence ratios considered in this study, acoustic wave starts to affect the flame in frequencies over $10 \mathrm{kHz}$. Therefore, for the application here, it is reasonable to neglect the acoustic wave effects even at frequencies as high as $2000 \mathrm{~Hz}$. Accordingly, the term associated with bulk viscosity in the viscous tensor can be neglected as well. The bulk viscosity should be 
considered for different compressible flow applications, such as shock/flame interactions, supersonic flames, or hypersonic flows [60].

Conservation of energy: [48]

$$
\begin{gathered}
\rho C_{p}\left(\frac{\partial T}{\partial t}\right)+\rho \boldsymbol{u} C_{p} \frac{\partial T}{\partial r}-\frac{1}{r} \frac{\partial}{\partial r}\left(r \lambda \frac{\partial T}{\partial r}\right)+\sum_{k=1}^{N_{s p}} h_{k} W_{k} \dot{\omega}_{k} \\
+\rho\left(\sum_{k=1}^{N_{s p}} C_{p, k} Y_{k} \bar{v}_{k}\right) \frac{\partial T}{\partial r}=0
\end{gathered}
$$

In this equation, $k$ is the species index which ranges over the $N_{s p}$ species; $T$, temperature; $Y_{k}$, mass fraction of species $k ; C_{p}$ and $C_{p, k}$ are the specific heats at constant pressure of the mixture and species $k$, respectively; $\lambda$ the mixture coefficient of thermal conductivity; $h_{k}$ the specific enthalpy of species $k ; W_{k}$ the molecular weight of species $k ; \dot{\omega}_{k}$ the molar production rate of species $k$; and $\bar{v}_{k}$ diffusion velocity of species $k$. Viscous work and the material derivative of pressure were neglected [61]. Radiation properties of non-premixed turbulent flames have been widely recognized as a significant component of heat transfer in combustion systems [62]. Nonpremixed flames radiate between $10 \%$ and $60 \%$ of their chemical energy release depending on fuel type. The radiation predominantly involves emission from soot, $\mathrm{CO}_{2}$, and $\mathrm{H}_{2} \mathrm{O}$ [62]. Conversely, in simulating turbulent premixed flames, the effects of radiation only become important when nitrogen reactions are a subject of study (due to the high-temperature sensitivity of NO kinetics), and when modeling laminar premixed flames near-limits (such as extinction) [63]. Some examples of including radiation effects in flame models are reduced cooling airflows in lean premixed combustors, miniaturization of combustors, and the possible use of radiation sensors in combustion 
control schemes. Therefore, in the application presented in this research, radiation was ignored without losing any significant effects on the results.

Balance of individual species mass:

$$
\rho \frac{\partial Y_{k}}{\partial t}+\rho \boldsymbol{u} \frac{\partial Y_{k}}{\partial r}+\frac{1}{r} \frac{\partial}{\partial r}\left(r \rho Y_{k} \bar{v}_{k}\right)-\boldsymbol{R}_{\boldsymbol{k}}=\mathbf{0}
$$

where $\boldsymbol{R}_{k}$ is the volumetric mass production rate of species $k$ due to potentially many chemical reactions.

The ideal gas equation of state completes the system:

$$
\rho=(P \bar{W} / R T)
$$

where $\bar{W}$ is the mean molecular weight of the mixture, and $R$ is the universal gas constant.

The low Mach number $(M a)$ assumption has been applied in this model which is valid for all applications in laminar flame modeling. This assumption asserts that the flow Mach number (the ratio of the fluid speed to the speed of sound in that fluid) is much less than unity. The pressure field $P$ in a gaseous flow consists of the static pressure field $p_{s}$ (the pressure of the fluid at rest which is the atmospheric pressure in this case) plus an additional pressure component associated with the velocity of the fluid $p_{d}$ (dynamic pressure). The ratio of dynamic and static pressure scales by the square of the Mach number (i.e., $\frac{p_{d}}{p_{s}} \propto M a^{2}$ ), and therefore in the low Mach number flows studied here $p_{s} \gg p_{d}$ or $M a \ll 1$. Thus, the total pressure can be approximated by the static 
pressure alone, as the dynamic pressure can be neglected, thus $P \approx p_{s}$. The static pressure or the total pressure, can be calculated using the ideal gas law.

$$
P \approx p_{s}=\frac{\rho R T}{\bar{W}}
$$

The flow with $M a^{2} \ll 1$ can be analyzed by standard perturbation methods which provide useful information on the solutions of hydrodynamically incompressible flows. In such flows, the density changes occur mainly by temperature changes, and pressure changes do not affect the density. This independency of pressure and density is usually what happens in typical laminar flames [14].

However, under fast transient conditions (high frequency oscillations or ignition), information that is communicated to downstream of the flow through acoustic pressure waves is faster than convective and diffusive transport [56]. When the governing equations are formulated based on an incompressible assumption, it states that the whole flow field responds to pressure waves instantly. In order to eliminate this assumption and allow the system to have a slower, and more physical response to a disturbance, the pressure distribution is required to be considered in the dynamics of the flow. Keeping the pressure as a dependent variable in the momentum equation is an appropriate way to eliminate the aforementioned assumption. The excess or dynamic pressure, $p_{d}=P-p_{s}$ is now included in the total pressure in the dependent variables which, in general, is a function of both time and radial location in an open burner configuration such as that of the current model.

This code has been developed for modeling premixed flames in the laminar regime, where the oscillations in mass flow rate (inlet velocity) are smaller than the flame burning rate at steady 
state, and also the flow time scale is greater than the chemistry time scale. As explained previously, the low Ma number assumption is a reasonable approximation in modeling laminar flames. However, when the frequency of oscillation becomes on the order of $10 \mathrm{kHz}$, the low Ma number assumption fails and the flame should be modeled as compressible flow. In that condition, the acoustic wave effects should be considered.

\subsubsection{Diffusion Model}

\subsubsection{The Mixture-Averaged Diffusion Approximation}

It has been shown that using the mixture averaged diffusion approximation can result in less computational difficulties in calculating diffusion velocities among other available methods in the literature [8]. This approximation neglects pressure-gradient-driven diffusion, thermal diffusion, and binary species diffusion (the sum of the diffusive effects on the $k^{\text {th }}$ species caused by concentration gradients in all other species). In order to calculate the diffusion velocities, a Fickian description of diffusion with a mixture-averaged diffusion coefficient for the $k^{\text {th }}$ species, $D_{k m}$ is considered in this method. By implementing this model, the diffusion velocities can be estimated as:

$$
\bar{v}_{k}=-D_{k m} \frac{\nabla Y_{k}}{Y_{k}}+\sum_{k=1}^{N S P} D_{k m} \nabla Y_{k} \quad k=1, \ldots, N S P
$$

\subsubsection{Mass Conservation Constraints}

In this section, three mass conservation constraints that should be considered in modeling a laminar flame are explained. From the definitions of the mixture density and mass-averaged 
velocity in terms of the (partial) densities and flow velocities of the individual species, the following constraints can be concluded:

$$
\sum_{k=1}^{N_{S P}} Y_{k}=1, \quad \sum_{k=1}^{N_{S P}} Y_{k} \bar{v}_{k}=0
$$

The other mass conservation constraint is derived from the chemical source terms and is defined as follows:

$$
\sum_{k=1}^{N_{S P}} R_{k}=\sum_{k=1}^{N_{S P}} W_{k} \dot{\boldsymbol{\omega}}_{k}=0
$$

These constraints ensure that a net creation of mass or a net diffusion of mass relative to the mass-averaged bulk flow will not occur in the simulation. With these constraints, it is possible to indicate that the total mass conservation equation results from summing up all the species equations. When computing highly diluted flames with approximate transport coefficients, a common and accepted approach is to "lump" all the diffusion velocity variations into that of nitrogen as follows:

$$
\bar{v}_{N_{2}}=-\frac{1}{Y_{N_{2}}} \sum_{k=1}^{N_{S P}} Y_{k} \bar{v}_{k}
$$


This method will certainly satisfy the constraint. However, this approach cannot be applied to flames where nitrogen is not present in excess. A more general way of ensuring this constraint is satisfied is to add a correction velocity to the diffusion velocity of each species at each point in the flow field. The correction velocity is defined as:

$$
\bar{v}^{c}=-\sum_{k=1}^{N_{S P}} Y_{k} \bar{v}_{k}
$$

Note that in the case of using an appropriate known model to calculate these velocities, they would automatically satisfy the constraint and the correction velocity would disappear. In other words, this solution results from the mutual incompatibility of the diffusion velocities as calculated from common approximate formulas. It should be noted that in this simulation both approaches have been studied for a methane-air stoichiometric flame. Since both methods led to promising results and the second approach is more general and practical, the correction velocity method was implemented in the code.

\subsubsection{Thermodynamic Properties and Transport Coefficients}

In the detailed numerical simulation of combustion systems, the estimation of thermodynamic properties and transport coefficients is essential. The species and mixture specific heats and the species enthalpies are two thermodynamic properties in the aforementioned governing equations. In addition to thermodynamic properties, there are three required transport coefficients comprising the dynamic viscosity, the thermal conductivity, and the mixture-averaged diffusion coefficients. Transport coefficients are estimated based on an averaging process using 
the relevant parameters for each component in the mixture. This mixture-averaged approach has been the standard in combustion modeling due to its accuracy and efficiency in the CHEMKIN library. The original CHEMKIN library code was written at Sandia and published in two separate packages in the early 1980 s, one dealing with chemical kinetics and thermodynamics and the other with the calculation of transport properties $[64,65]$. In this thesis, the first package is referred as "CHEMKIN" and the second as "TRANSPORT".

Finding the solution variables from the governing equations (e.g., the temperature profile from the solution of the energy equation), requires the aforementioned mixture thermodynamic properties, and the mixture density, which is recovered from the ideal gas law ${ }^{1}$. One of these thermodynamic properties is the specific heat capacity at constant pressure for the $k^{\text {th }}$ species which depends only on temperature. The mixture specific heat at constant pressure is then taken as the mass average of the species specific heats. The specific enthalpies are given mathematically by a definite integral of the species specific heats over a prescribed temperature range; in CHEMKIN, however, these quantities are computed more efficiently as simple polynomial fits of the published data.

\subsubsection{Chemistry Models}

One of the major frontiers of combustion science is the generation of reliable detailed kinetic models for fuels, and expanding the application of such models to study challenging burning regimes in technologically complex systems. As mentioned before, the development of numerical codes that are able to incorporate these detailed chemistry models is essential.

\footnotetext{
${ }^{1}$ This has been done through the density subroutine in CHEMKIN library
} 
The problem includes the size of the models and the amount of computation required, and the wide difference of time scales supported by them, which leads to the problem of stiffness. The reaction set of an arbitrarily complex, detailed chemistry model can be written in symbolic form as:

$$
\sum_{k=1}^{N_{s p}} \dot{v}_{k i} \chi_{k} \rightleftharpoons \sum_{k=1}^{N_{s p}} \ddot{v}_{k i} \chi_{k}, \quad i \in \mathcal{R}
$$

where $k$ and $i$ are the species and reaction indices, respectively; $\dot{v}_{k i}$ and $\ddot{v}_{k i}$, the stoichiometric coefficients of reactant $k$ in both forward and reverse reaction $i ; \chi_{k}$, the chemical symbol of species $k$; and $\mathcal{R}$, the set of $N_{\text {reac }}$ reactions. A rate of progress $Q_{i}$ may be defined for each elementary reaction $i$ according to the law of mass action, and the sum of the rates of progress for all reactions involving species $k$, multiplied by the appropriate net stoichiometric coefficient, gives the molar production rate for that species:

$$
\begin{gathered}
Q_{i}=k_{i}^{f}(T) \prod_{k=1}^{N_{s p}}\left(\frac{\rho Y_{k}}{W_{k}}\right)^{\dot{v}_{k i}}-k_{i}^{r}(T) \prod_{k=1}^{N_{s p}}\left(\frac{\rho Y_{k}}{W_{k}}\right)^{\ddot{v}_{k i}} \\
\dot{\omega}_{k}=\sum_{i \in R}\left(\ddot{v}_{k i}-\dot{v}_{k i}\right) Q_{i}
\end{gathered}
$$

$k_{i}^{f}$ and $k_{i}^{r}$ are the forward and reverse rate constants for reaction $i$, respectively. These constants strongly depend on temperature. The relationship for the forward coefficient is modeled by a modified Arrhenius expression: 


$$
k_{i}^{f}(T)=A_{i} T^{\beta_{i}} \exp \left(-E_{i} / R T\right)
$$

In Eq. (2.15), the coefficient $A_{i}$, the temperature exponent $\beta_{i}$, and the activation energy $E_{i}$, are usually quantified by the kinetic mechanism experimentally. Then the results are tuned as needed to achieve the correct prediction of premixed laminar flame speeds or ignition and extinction criteria. Although it is possible to specify Arrhenius parameters separately for the reverse reactions, the $\mathrm{CHEMKIN}^{2}$ [64] default is to back out the reverse rate constant from the forward rate constant and the equilibrium constant:

$$
k_{i}^{r}=\frac{k_{i}^{f}}{K_{c i}}
$$

The equilibrium constant is determined from a thermodynamic calculation. Although $K_{c i}$ is given in concentration units, the equilibrium constants are more easily determined from the thermodynamic properties in pressure units; they are related by:

$$
K_{c i}=K_{p i}\left(\frac{P_{a t m}}{R T}\right)^{\sum_{k=1}^{K} v_{k i}}
$$

The equilibrium constants $K_{p i}$ are obtained with the relationship:

${ }^{2}$ CHEMKIN is a software package and its purpose is to facilitate the formation, solution, and interpretation of problems involving elementary gas-phase chemical kinetics. 


$$
K_{p i}=\exp \left(\frac{\Delta S_{i}^{0}}{R}-\frac{\Delta H_{i}^{0}}{R T}\right)
$$

The $\Delta$ refers to the change that occurs in passing completely from reactants to products in the $i^{t h}$ reaction. The reason for this reliance on the equilibrium constant is due to the fact that the Arrhenius model is naturally empirical, the formula for which is well founded from a theoretical point of view [8]. The mechanism used in all the simulations in this thesis, includes 36 species and 219 reactions.

\subsection{Numerical Model}

A standard solution approach for such flow problems is to use staggered grids in the context of finite-volume methods [66]. Therefore, a finite volume formulation was derived for the onedimensional total mass, momentum, energy, and species equations. The boundary conditions were based on the underlying physical characteristics of the model. For the inlet boundary (burner surface):

$\dot{m}=\dot{m}_{0}+B \sin (\omega t)$, specified mass flow (for steady state $B=0$ )

$d p / d r=0$, zero gradient for pressure

$T=T_{u}$ (unburned mixture temperature)

$Y_{k}=Y_{k, u}$ (unburned mixture conditions)

and for the outlet boundary conditions:

$d \dot{m} / d r=0$, zero gradient for mass flow rate $(d(\rho u A) / d r=0)$

$p=P_{\text {atm }}$, atmospheric pressure

$d T / d r=0$, zero gradient for temperature 
$d Y_{k} / d r=0$, zero gradient for species mass fractions were applied, assuming that the boundary was far enough from the flame that the flow composition is no longer appreciably changing.

\subsubsection{Discretization Techniques}

The grid stencil and boundary conditions for individual dependent variables are explained in this section. All dependent variables are represented at the control-volume center nodes, except the radial velocity which is represented at the control-volume faces. The spatial discretization uses the finite-volume method for the uniform grid system. For the momentum, energy, and species equations, the diffusive terms were discretized using a second-order central difference scheme while the convective terms were discretized using the exact exponential solution to approximate the values between the centers of two control volumes [67].

In order to discretize the governing equations based on the finite volume method, the vector and the integral form of these equations were used.

Conservation of total mass:

$$
\frac{\partial \rho}{\partial t}+\nabla \cdot \rho \boldsymbol{u}=0
$$

Conservation of momentum:

$$
\frac{\partial \rho \boldsymbol{u}}{\partial t}+\nabla \cdot \rho \boldsymbol{u} \boldsymbol{u}=\nabla . \boldsymbol{S}+\boldsymbol{f}
$$


Conservation of energy:

$$
\frac{\partial \rho e}{\partial t}+\nabla \cdot \rho \boldsymbol{u} e=\nabla \cdot(\boldsymbol{u} . \boldsymbol{S})-\nabla \cdot \boldsymbol{q}+\boldsymbol{u} \cdot \boldsymbol{f}+\boldsymbol{Q}
$$

Balance of individual species mass:

$$
\frac{\partial \rho Y_{k}}{\partial t}+\nabla \cdot \rho Y_{k} \boldsymbol{u}=-\nabla \cdot \boldsymbol{j}_{\boldsymbol{k}}+\boldsymbol{R}_{\boldsymbol{k}}
$$

In these governing equations; $S$ is stress tensor; $\boldsymbol{f}=\sum_{k=1}^{N_{S p}} f_{k}$, body force vector, where the force can in theory vary depending on the chemical species (e.g., due to electromagnetic fields in a plasma), or it will simply be a constant force due to gravity (g); however, in this work, this term is neglected; $e$, specific total energy (chemical, sensible, and kinetic); $\boldsymbol{q}$, diffusive energy flux vector; $\boldsymbol{Q}$, volumetric energy source (e.g., due to an ignition source or a laser), to be ignored in this work; $\boldsymbol{j}_{k}$, diffusive mass flux of species $k ; \boldsymbol{R}_{k}$, volumetric mass production rate of species $k$ due to chemical reaction; $\bar{W}$, mean molecular weight of the mixture; and $R$, universal gas constant. The symbol $\nabla$ is the vector derivative operator.

Since the fluids used in this research and many similar studies are known to be Newtonian, the stress-strain relation is considered approximately linear. For such fluids, the components of the vector momentum conservation equation are known as the Navier-Stokes equations [8] and the stress tensor has the following familiar form: 


$$
\begin{gathered}
\boldsymbol{S}=-p \boldsymbol{I}+\boldsymbol{T} \\
\boldsymbol{T}=\mu\left((\nabla \boldsymbol{u})+(\nabla \boldsymbol{u})^{T}\right)-\frac{2}{3} \mu \nabla \cdot \boldsymbol{u} \boldsymbol{I}
\end{gathered}
$$

which is the sum of the isotropic pressure tensor and the viscous stress tensor $(\boldsymbol{T}) . \mu$ is the dynamic viscosity of the mixture which, like the other transport coefficients, is a function of the thermodynamic state of the mixture, and $\boldsymbol{I}$ is the unit tensor. The diffusive fluxes of energy and mass are given as follows:

$$
\begin{gathered}
q=-\lambda \nabla T+\sum_{k=1}^{N_{S P}} h_{k} \dot{j}_{k}+q_{r a d} \\
j_{k}=\rho Y_{k} \bar{v}_{k}
\end{gathered}
$$

The radiative flux $\left(q_{r a d}\right)$ is neglected in this work. The Dufour effect, whereby an energy flux arises from concentration gradients, is typically negligible in high-heat-release combustion processes, and hence is omitted above. The energy equation is transformed into an equation for temperature using various thermodynamic relationships for energy and enthalpy in laminar flames. The result is presented in Eq. (2.27).

$$
\rho C_{p} \frac{D T}{D t}=\dot{\omega}_{T}+\frac{D p}{D t}+\nabla \cdot(\lambda \nabla T)-\left(\rho \sum_{k=1}^{N_{S P}} C_{p, k} Y_{k} \boldsymbol{u}_{k}\right) \cdot \nabla T-\nabla \cdot q_{r a d}+T: \nabla \boldsymbol{u}
$$


In Eq. (2.27), $\dot{\omega}_{T}=-\sum_{k=1}^{N_{s p}} h_{k} W_{k} \dot{\omega}_{k}$ the heat source due to chemical reaction; and $D / D t$ denotes the material derivative. In this equation, the terms involving the heat due to viscous effects in the fluid (friction) and the material derivative of pressure, related to mechanical work, are negligible for low speed unconfined flows [61].

The code solves for $p, u, T, Y_{k}$ as dependent variables and density $\rho$ which is determined by Eq. (2.5) when necessary. The transient term in the continuity Eq. (2.1) is rewritten in terms of the actual solution variables as in [25] because density is not one of the dependent variables:

$$
\frac{\partial \rho}{\partial t}=\frac{\rho}{P} \frac{\partial P}{\partial t}-\frac{\rho}{T} \frac{\partial T}{\partial t}-\rho \bar{W} \sum_{k=1}^{N S P}\left(\frac{1}{W_{k}} \frac{\partial Y_{k}}{\partial t}\right)
$$

The continuity equation is spatially first order and only neutrally stable; therefore, an artificial damping term of the form $\sigma \frac{\Delta r}{u_{0}}\left(\frac{\partial^{2} p}{\partial r^{2}}\right)$ is introduced to maintain numerical stability. In this additional term, $u_{0}$ is a reference velocity and $\sigma$ is a coefficient which should be chosen appropriately. In order to guarantee that the solution is not affected by this diffusive term, $\sigma=$ $10^{-3}$ was found acceptable without affecting the solution accuracy [56]. Consequently, the final form of the mass conservation equation is given by:

$$
\rho\left(\frac{1}{P} \frac{\partial p}{\partial t}-\frac{1}{T} \frac{\partial T}{\partial t}-\bar{W} \sum_{k=1}^{N S P}\left(\frac{1}{W_{k}} \frac{\partial Y_{k}}{\partial t}\right)\right)+\frac{1}{r} \frac{\partial}{\partial r}(\rho \boldsymbol{u} r)-\frac{\sigma \Delta r}{2 u_{0}} \frac{\partial^{2} p}{\partial r^{2}}=0
$$

All of the above governing equations can be presented in integral form: 


$$
\frac{d}{d t} \iiint_{d \forall} \rho \emptyset d \forall+\iint_{d s}(\rho \emptyset \vec{V} \cdot \hat{n}-\nabla \cdot \Gamma \nabla \emptyset) d s=\iiint_{d \forall} \kappa d \forall
$$

where $\emptyset$ is the conserved variable, $\aleph$ is the source term, $\Gamma$ is the diffusion parameter, $s$ is the surface area of the control volume, and $\forall$ is the control volume. All finite volume methods require derivation of the discretization equations directly from the integral form, applied to an arbitrary control volume. The control volume considered in this model is shown in Fig. (2.3), where $\mathbf{P}$ is the centre of the control volume.
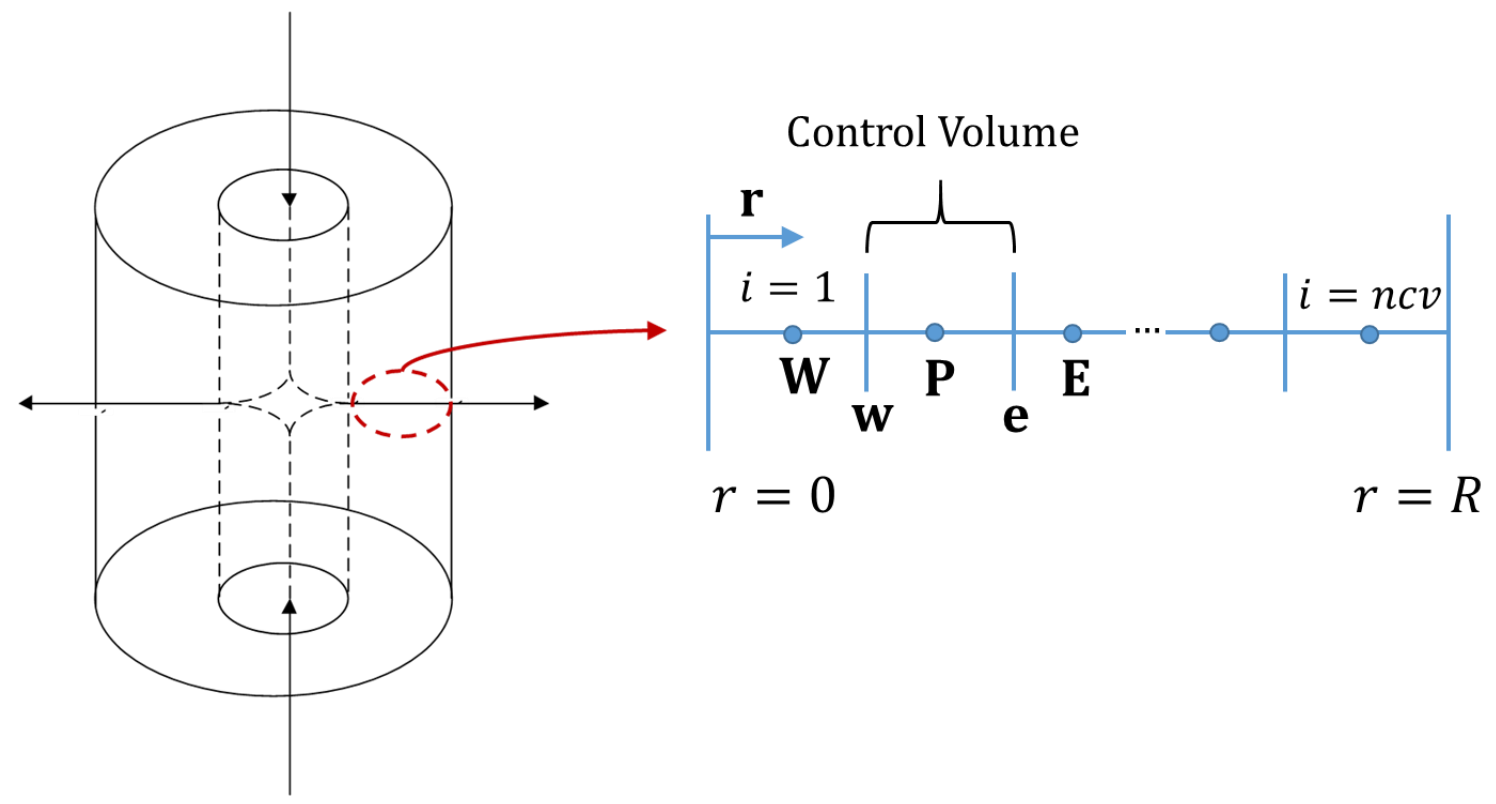

Fig. 2.3. Computational domain starting from the burner surface (inner cylinder, $r=0$ ) to outer boundary (outer cylinder $r=R$ )

For instance, discretizing the temporal terms can be illustrated as follows: 


$$
\begin{gathered}
\frac{\partial \rho}{\partial t} \rightarrow \int_{w}^{e} \int_{t}^{t+\Delta t} \frac{\partial \rho}{\partial t} d t d A \rightarrow\left[\rho_{\boldsymbol{P}}^{n+1}-\rho_{\boldsymbol{P}}^{n}\right] v o l_{\boldsymbol{P}} \\
\frac{\partial \rho u}{\partial t} \rightarrow \int_{\boldsymbol{P}}^{t} \int_{t}^{t+\Delta t} \frac{\partial \rho u}{\partial t} d t d A \rightarrow\left[\rho_{e}^{n+1} u_{e}^{n+1}-\rho_{e}^{n} u_{e}^{n}\right] v o l_{e} \\
\frac{\partial \rho T}{\partial t} \rightarrow \int_{w}^{e} \int_{t}^{t+\Delta t} \frac{\partial \rho T}{\partial t} d t d A \rightarrow\left[\rho_{\boldsymbol{P}}^{n+1} T_{\boldsymbol{P}}^{n+1}-\rho_{\boldsymbol{P}}^{n} T_{\boldsymbol{P}}^{n}\right] v o l_{\boldsymbol{P}} \\
\frac{\partial \rho Y_{k}}{\partial t} \rightarrow \int_{w}^{e} \int_{t}^{t+\Delta t} \frac{\partial \rho Y_{k}}{\partial t} d t d A \rightarrow\left[\rho_{\boldsymbol{P}}^{n+1} Y_{k}^{n+1}-\rho_{\boldsymbol{P}}^{n} Y_{k}^{n}\right] v o l_{\boldsymbol{P}}
\end{gathered}
$$

And the diffusion terms can be discretized (second order discretization) from the integral form:

$$
\nabla . \sigma \nabla P \rightarrow \int_{t}^{t+\Delta t} \int_{w}^{e} \nabla \cdot \sigma \nabla P d r d t \rightarrow\left[\sigma_{e} A_{e} \frac{P_{E}-P_{\boldsymbol{P}}}{\Delta r}-\sigma_{w} A_{w} \frac{P_{\boldsymbol{P}}-P_{W}}{\Delta r}\right]^{n+1} \Delta t
$$




$$
\begin{gathered}
\left.-\frac{4}{3} \mu\left[\nabla \cdot(\nabla u)+\frac{2}{r} \nabla u-\frac{2 u}{r^{2}}\right)\right] \\
\left.\quad \rightarrow \int_{t}^{t+\Delta t} \int_{P}^{E}\left(-\frac{4}{3} \mu\left[\nabla \cdot(\nabla u)+\frac{2}{r} \nabla u-\frac{2 u}{r^{2}}\right)\right]\right) d r d t \\
\rightarrow-\frac{4}{3} \mu\left(\left[A_{E} \frac{u_{e e}-u_{e}}{\Delta r}-A_{P} \frac{u_{e}-u_{w}}{\Delta r}\right]^{n+1}+\left[\frac{2}{r} \frac{u_{E}-u_{P}}{\Delta r}\right]^{n+1}\right. \\
\left.\quad-\left[\frac{2 u_{e}}{r^{2}}\right]^{n+1}\right) \Delta t \\
\nabla . \lambda \nabla T \rightarrow \int_{t}^{t+\Delta t} \int_{w}^{e} \nabla \cdot \lambda \nabla T d r d t \rightarrow\left[\lambda_{e} A_{e} \frac{T_{E}-T_{P}}{\Delta r}-\lambda_{w} A_{w} \frac{T_{P}-T_{W}}{\Delta r}\right]^{n+1} \Delta t \\
\nabla .\left(\rho Y_{k} \bar{v}_{k}\right) \rightarrow \int_{t}^{t+\Delta t} \int_{w}^{e} \nabla \cdot\left(\rho Y_{k} \bar{v}_{k}\right) d r d t \rightarrow\left[A_{e}\left(\rho Y_{k} \bar{v}_{k}\right)_{e}-A_{w}\left(\rho Y_{k} \bar{v}_{k}\right)_{w}\right]^{n+1} \Delta t
\end{gathered}
$$

And the convective terms can be discretized (second order discretization) from the integral form:

$$
\begin{gathered}
\nabla . \rho u \rightarrow \int_{t}^{t+\Delta t} \int_{w}^{e} \nabla . \rho u d r d t \rightarrow\left[(\rho u A)_{e}-(\rho u A)_{w}\right]^{n+1} \Delta t \\
\nabla . \rho u u \rightarrow \int_{t}^{t+\Delta t} \int_{P}^{E} \nabla . \rho u u d r d t \rightarrow\left[(\rho u u A)_{E}-(\rho u u A)_{P}\right]^{n+1} \Delta t
\end{gathered}
$$




$$
\begin{gathered}
\nabla . \rho u T \rightarrow \int_{t}^{t+\Delta t} \int_{w}^{e} \nabla \cdot \rho u T d r d t \rightarrow\left[(\rho u T A)_{e}-(\rho u T A)_{w}\right]^{n+1} \Delta t \\
\nabla . \rho u Y_{k} \rightarrow \int_{t}^{t+\Delta t} \int_{w}^{e} \nabla \cdot \rho u Y_{k} d r d t \rightarrow\left[\left(\rho u Y_{k} A\right)_{e}-\left(\rho u Y_{k} A\right)_{w}\right]^{n+1} \Delta t
\end{gathered}
$$

All of the source terms are calculated on the centre of the control volume $(\mathbf{P})$.

\subsubsection{Solution Algorithm}

\subsubsection{Modified Newton's Method}

Once the coupled, nonlinear system of equations, shown in the previous sections, has been discretized based on finite volume methods, a solution should be implemented on the onedimension grid for all of the dependent variables. The problem contains a total of $\mathrm{N}_{\mathrm{dep}}$ dependent

variables at each grid point. In this method, $\overrightarrow{\mathrm{X}}_{\mathrm{ii}=0,1,2, . .}^{\mathrm{n}=0,1,2,}$ is the array of all unknowns (dependent variables) at time step $n$ and Newton's iteration $i i$ at all the control volumes $j j$, which is equal to $\mathrm{N}_{\mathrm{cv}} \times \mathrm{N}_{\mathrm{dep}}$. The complete vector of $\overrightarrow{\mathrm{X}}$ is shown below:

$$
\vec{X}=\left[P_{1}, u_{1}, T_{1}, Y_{1,1}, \ldots, Y_{k, 1}, \ldots, P_{j}, \ldots, u_{j}, \ldots, T_{j}, \ldots, Y_{1, j}, \ldots, Y_{k, j}\right]^{T}
$$

The discretized equations (continuity, momentum, energy and mass species conservation equations) are presented in residual form as follows: 


$$
\overrightarrow{\mathcal{F}}(\vec{X})=0
$$

The system is solved iteratively using Newton's method at each time step using the initial guess $\overrightarrow{\mathrm{X}}_{0}$, in which the $i^{\text {th }}$ iteration takes the form:

$$
\overrightarrow{\mathrm{X}}_{\mathrm{i}+1}=\overrightarrow{\mathrm{X}}_{\mathrm{i}}+\Delta \overrightarrow{\mathrm{X}}
$$

where $\Delta \overrightarrow{\mathrm{X}}$ is calculated as follows:

$$
[\mathrm{J}][\Delta \overrightarrow{\mathrm{X}}]=-\overrightarrow{\mathcal{F}}
$$

Here, $\vec{J}$ is the Jacobian matrix with entries defined as:

$$
\mathrm{J}_{\mathrm{i}, \mathrm{j}} \approx \frac{\mathcal{F}_{\mathrm{i}}\left(\mathrm{X}_{\mathrm{j}}+\delta\right)-\mathcal{F}_{\mathrm{i}}\left(\mathrm{X}_{\mathrm{j}}\right)}{\delta}
$$

where

$$
\delta=\mathrm{r} \times \overrightarrow{\mathrm{X}}_{\mathrm{j}}+\mathrm{a}
$$

and where the relative and absolute perturbations, $r$ and a are chosen to be the square root of the computer's unit round off $\left(8.4 \times 10^{-8}\right)$. The Newton iteration continues until the maximum norm of $\Delta \overrightarrow{\mathrm{X}}$ is reduced to within a user-specified tolerance. Newton's method is considered to have 
converged when the scaled 2-norm of the $K^{\text {th }}$ update vector is below a specified tolerance (Eq. (2.49)). In the convergence criterion for Newton's method, $S F_{j}$ is the scale factor of the variable associated with the $j^{\text {th }}$ value of $\overrightarrow{\mathrm{X}}$. The convergence criteria was selected as $10^{-3}$ to $10^{-4}$, which will serve to provide 3 to 4 significant digits in each computed value [68]. In this study, the relative tolerance is kept at $10^{-4}$. If the solution is converging within a few Newton's iterations, as determined by theoretical estimates [69], then the Jacobian can be reused for the next iteration without substantially degrading progress towards a converged solution. This enhancement is known as 'modified' Newton's method [17]. Since the most time-consuming part of this computation is Jacobian matrix formation, reducing the number of Jacobian evaluations makes the simulation much faster.

$$
\frac{\sqrt{\sum_{j=1}^{N_{d e p} \times N_{c v}\left(\frac{X_{j}^{K+1}-X_{j}^{K}}{S F_{j}}\right)^{2}}}}{N_{d e p} \times N_{c v}} \leq \text { Tol }
$$

\subsubsection{Linear System Solution}

The linear system in Eq. (2.46) should be solved in every Newton's method iteration. In this model, an iterative technique, the bi-conjugate gradient stabilized method (Bi-CGSTAB) linear solver [70] with a block Gauss-Seidel pre-conditioner is applied [71]. Bi-CGSTAB, which is developed for the numerical solution of non-symmetric linear systems, is a variant of the biconjugate gradient method (Bi-CG) and has faster and smoother convergence than the original $\mathrm{Bi}$ $\mathrm{CG}$ as well as other variants such as the conjugate gradient-squared method (CG-S). Therefore, 
Bi-CGSTAB produces more accurate residual vectors compared to the CG-S and it is very competitive with other non-symmetric linear systems solvers such as GMRES [72].

Since the problem was solved with dimensional variables, as opposed to their nondimensional counterparts, it is necessary to discuss the actual physical size of the computational domain. The naturally occurring length scale of this problem is the thickness of the flame, which is expected to be of the order of $3 \mathrm{~mm}$. Consequently, the calculated results could be expected to depend on the radius of curvature of the flame if it were of the same order of magnitude as the flame thickness. To avoid these size dependencies, the inflow boundary was set to be $10 \mathrm{~mm}$ (burner surface) such that the radius of curvature of the flame $(35 \mathrm{~mm})$ was at least 10 times that of its thickness $(3 \mathrm{~mm})$.

\subsection{Computational Results and Performance}

\subsubsection{Ignition}

The first step to start the simulation is to ignite the reactants. To reach a steady state solution, the lean reactant mixture with equivalence ratio of 0.8 at $300 \mathrm{~K}$ was introduced into pure nitrogen at $1800 \mathrm{~K}$ (nitrogen is inert in this model), which has initially filled the computational domain. The mass flow rate in this simulation is $0.0725 \mathrm{~kg} / \mathrm{s} . \mathrm{m}$. The temperature and species fields evolve until they reach steady state. It is worth pointing out that this stationary flame was solved by time stepping the transient model with steady boundary conditions stated in section 2.3 . In the beginning of the simulation, the time step was kept small enough (e.g. $10^{-6}$ s) to avoid convergence difficulties and also to maintain accuracy. Once the flame is formed, the time step is increased by the factor of 0.1 at each time iteration until it gets to $10^{-5} \mathrm{~s}$. Figure 2.3 illustrates how the temperature field and reactants mass fractions form initially upon ignition and move towards 
steady state for the specific case of an equivalence ratio equal to 0.8. (Equivalence ratio is a nondimensional measure of the relative amounts of fuel and air such that a value of 1.0 is a stoichiometric mixture, and values less that one have excess air in the reactants.) As separated by different line colours, the first three lines (blue) in all plots show that the temperature and the concentration of the reactants (methane and oxygen) are increasing without any reactions occurring. In the next 4 time steps (shown by red lines), the reactions take place and the temperature rises while the concentrations of the reactants drop. In the final stage of this process, as depicted by the green lines, the profiles are formed and they are only moving away from the burner with minor changes to reach the steady state condition (black line). 

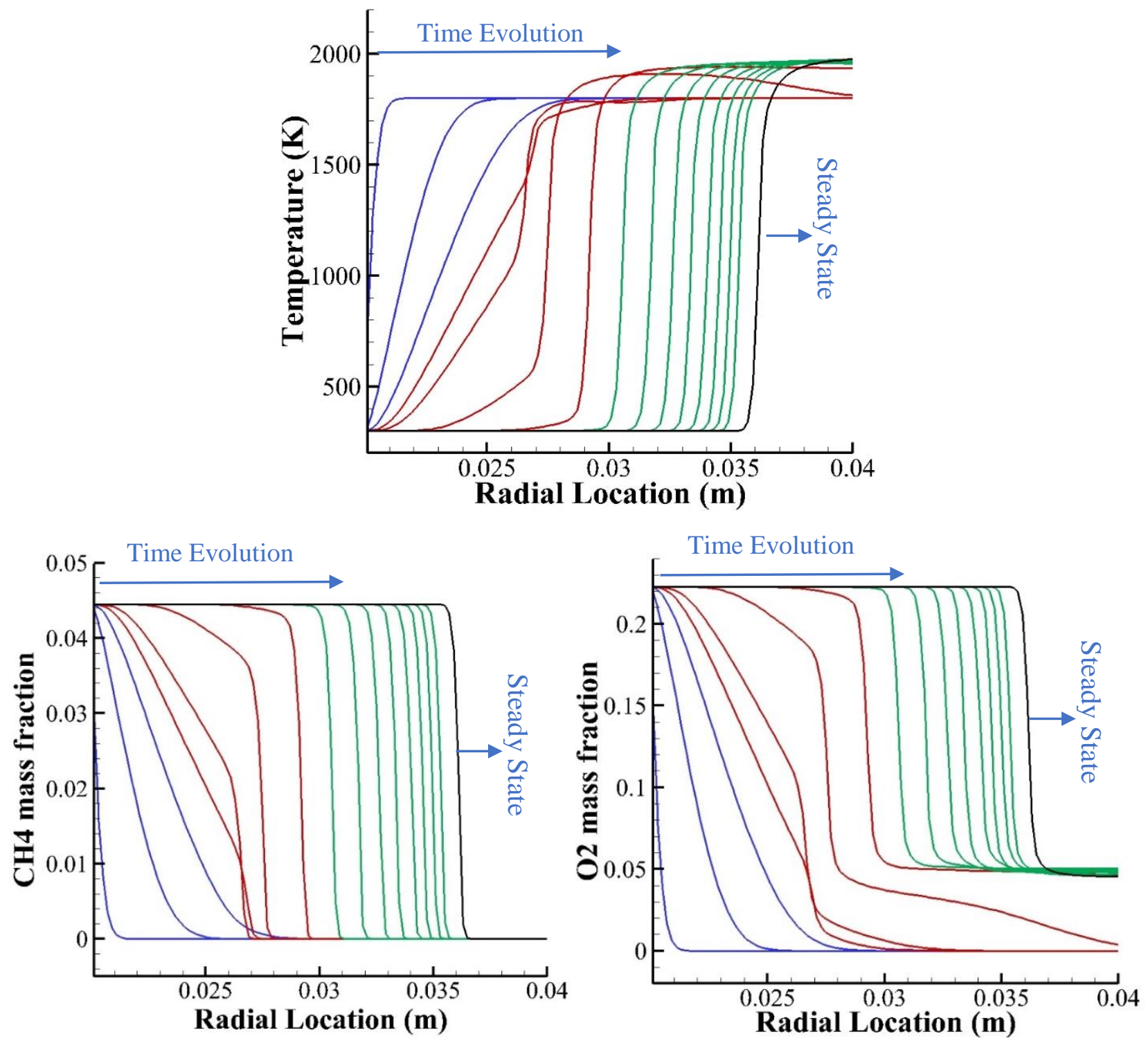

Fig. 2.4 Evolution of temperature field and reactants mass fractions from ignition to steady state at different times

As can be observed in Fig. 2.4, during the first three time steps in Fig. 2.3, when the reactions have not taken place yet, no products are formed. Starting from the next time step, major products can be seen in the domain. After the first molecule of the reactants is cracked, radicals (minor species) are formed in the domain (Fig. 2.5), and the intermediate reactions occur. 

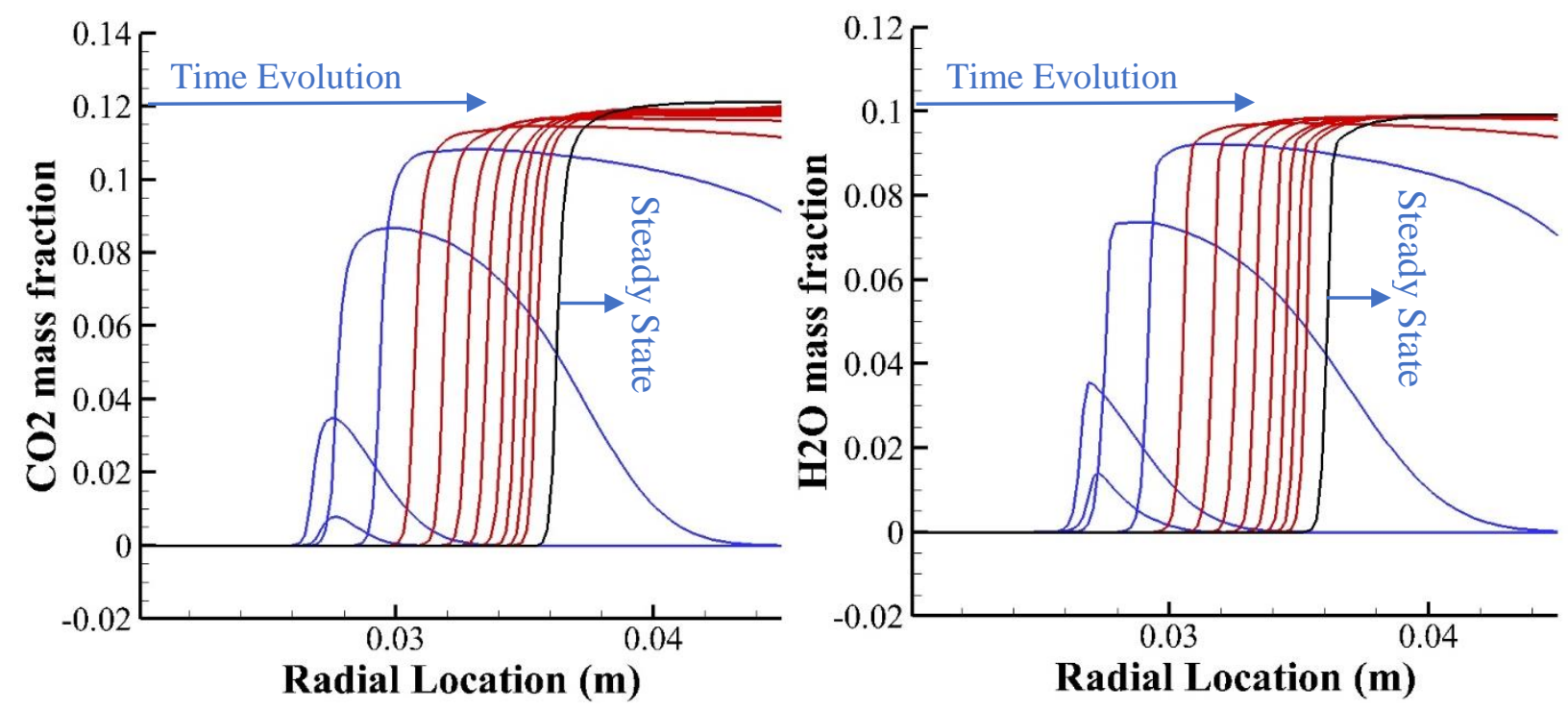

Fig. 2.5 Evolution of products mass fractions from ignition to steady state condition at different times

In Fig. 2.5, among 31 minor species, 6 of them are illustrated due to their importance in reaction mechanism and analyzing a flame structure. Also, in experimental studies, the concentration of these species are usually measured and reported. As can be seen in Fig. 2.5, due to the fast production and consumption, the concentration of these species is significantly lower than the other major species in the reaction layer. 

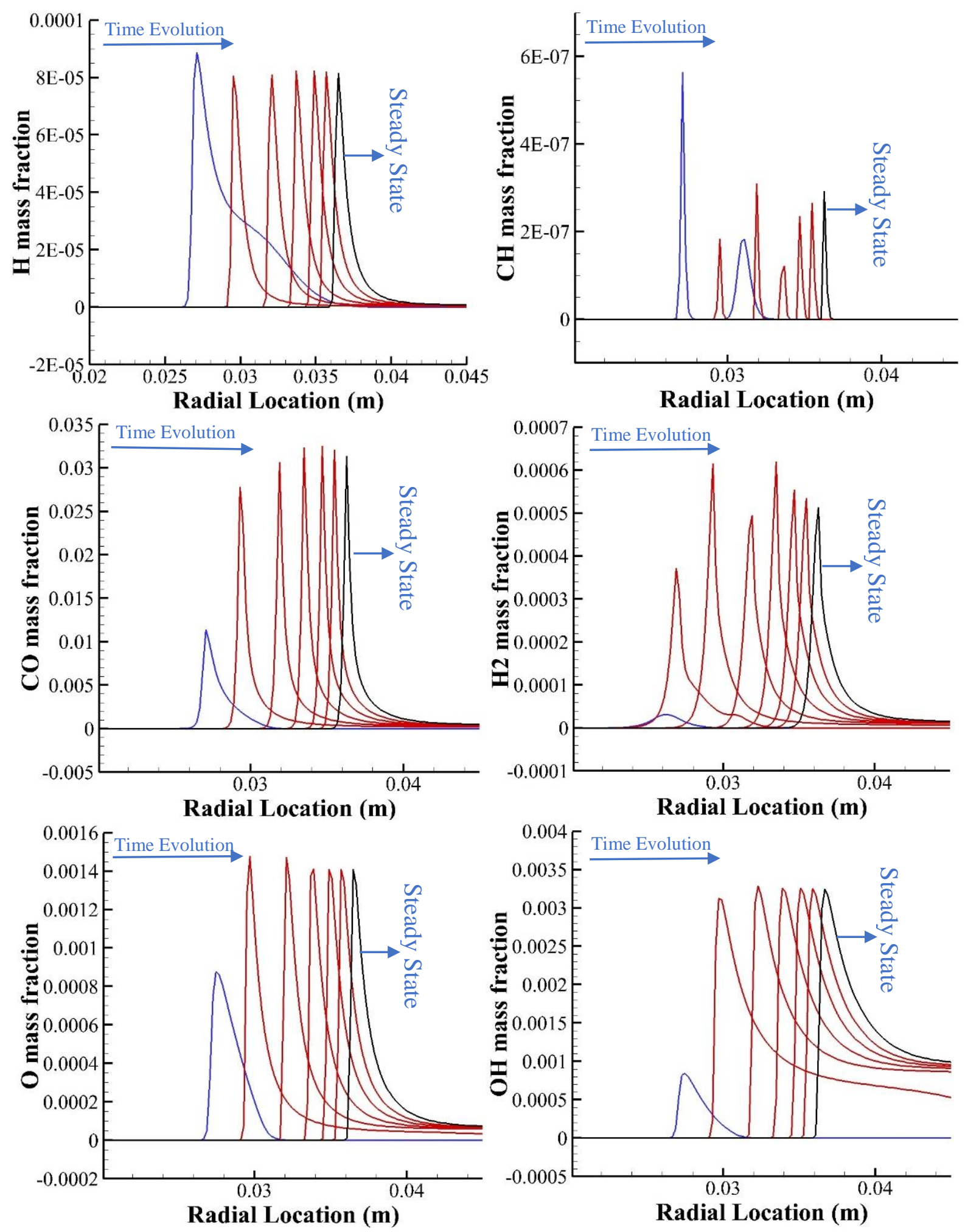

Fig. 2.6 Evolution of minor species mass fractions from ignition to steady state condition in 6 different times 


\subsubsection{Steady State Conditions}

Once the difference between the last two time steps reaches a proper value, the last solution was saved as the steady state result. The important variables are also saved separately to be plotted if needed. In order to ensure the validity of the solution, two different methods can be used. In the first method, the experimental burning velocity of a laminar premixed flame from the literature is compared to the burning rate of the simulated flame. In the second method, the equilibrium concentration of the final species on the product side can be compared to the values reported in the literature. These methods are explained in more detail in the next chapter (chapter 3). It should be noted that comparison to experimental results in an identical geometrical configuration is not possible due to a lack of information in literature. Setting up such a burner and running an experiment in this geometry is prohibitively challenging due to the buoyancy effects.

\subsubsection{Transient Boundary Condition}

Once the steady state solution is obtained, it can be used as an initial condition for a transient boundary case. As mentioned earlier in the introduction, different transient boundary conditions such as sinusoidal variation of mass flowrate (inlet velocity), pulse, ramp, or step functions could be applied to this code. The transient response of laminar flames to these functions are important due to their similarities to flow perturbations upstream of a turbulent flame front. The example of using a sinusoidal function as an inlet boundary condition of mass flow rate is presented in the next chapter.

\subsection{Summary}

In this chapter, a numerical algorithm has been developed in order to simulate a onedimensional time dependent laminar premixed flame in radial coordinates. The importance of 
developing this code in combustion research is to seek further understanding of the effect of stretch (transient effects of a curved flame front) on premixed flames by considering laminar flame dynamics in a cylindrically-symmetric outward radial flow geometry (i.e., inwardly propagating flame). This numerical model was designed to study the flame response when the flow and scalar fields align (i.e., no tangential strain on the flame) while the flame either expands (positive stretch) or contracts (negative stretch, which is a case that has been seldom explored) radially. First, for a specific equivalence ratio, a steady state solution was obtained using time stepping and steady boundary conditions. In the next step, by changing the boundary conditions to transient, the steady flame can be introduced as the initial condition in order to study the flame behaviour in an oscillating flow. The transient results are presented in the next chapter, where a comparison has been made between an unstretched stationary flame and stretched expanding and contracting flames. The unique aspect of this code is that the flame is subjected to both positive and negative stretch while the effect of strain rate is minimized.

With regard to an inwardly-propagating premixed cylindrical flame that is forced to radially expand and contract, this numerical study has been applied to revisit the quasi-steady assumptions for laminar flamelet models when the flame is subjected to both positive and negative stretch. Therefore, this code can be used to study the effects of different parameters such as mean curvature, frequency, amplitude, etc. on local burning rate of laminar premixed flames. The results can be used in order to capture each specific flame behaviour in a simple mathematical relation using a transfer function, as will be demonstrated in the coming chapters. 


\section{Chapter 3: A Transient Response of a Laminar Premixed Flame to a Radially Diverging/Converging Flow}

This chapter is based on the following published paper:

Meysam Sahafzadeh, Larry W. Kostiuk, Seth B. Dworkin, "Transient response of a laminar premixed flame to a radially diverging/converging flow," Combustion and Flame, vol. 179, pp. 51-62, May 2017.

\subsection{Introduction}

In premixed turbulent combustion, the local flame experiences both positive and negative stretch. Kostiuk and Bray [5] studied the distribution of stretch rates on the flamelet surface; it was shown that in an outwardly propagating spherical flame, $30 \%$ to $50 \%$ of the flame is under compression (i.e., negative stretch rates) at any time. Therefore, modeling the region of negative stretch rates in terms of consumption velocity and analyzing the mean effects of stretch rate on conversion from reactants to products is of importance. However, as mentioned previously, the geometry considered in the literature to analyze flamelets, namely, counter-flow flame configurations, only involves positive stretch.

In this chapter, through the consideration of an inwardly-propagating premixed cylindrical flame that is forced to radially expand and contract, a numerical investigation has been made to revisit the quasi-steady assumptions for laminar flamelet models when the flame is subjected to both positive and negative stretch. Furthermore, since the flame stretch in this work is due to the motion of a curved flame, the second term in Eq. (1.2) is zero and the effects of tangential strain are eliminated. Finally, in order to study the effect of equivalence ratio on transient response of a 
laminar flame to a periodic flow, two fuel lean flames are compared with the stoichiometric condition.

In premixed turbulent combustion, the time-varying vortices stretch the flamelets and cause small curvature on flame fronts; therefore, generating libraries to describe the flamelets based on steady laminar stagnation point flames (planar strained flames) has been challenged in some ways [73]. One of these challenges is related to the instantaneous structure of a turbulent flame wherein the flame front includes positive stretch and negative stretch at certain locations. The planar strained flames, which are only positively stretched, are not capable of describing the concave curved flame fronts towards the fresh gases. As has been mentioned earlier, most premixed turbulent flames involve negative stretch and highly curved flame fronts, therefore, to generate a more rigorous flamelet library to model a premixed turbulent flame, in addition to a counter-flow configuration, the time-dependent motion of a curved flame should be included. The model that has been proposed and developed in the previous chapter enables the study of this aspect of flamelets and the inclusion of the negative stretch in the computations of a laminar premixed flame.

\subsection{Flame Geometry and Numerical Model Specification}

The model developed in the previous chapter was used to create a one-dimensional radially (cylindrical) outward flow of a pre-mixture of methane and air (comprising oxygen and nitrogen) in stoichiometric composition and ambient temperature (Fig. 2.1). The radius of the burner is 5 $\mathrm{cm}$ and the mass flowrate for the stoichiometric condition is considered to be $0.2 \mathrm{~kg} / \mathrm{s} . \mathrm{m}$. 


\subsubsection{Numerical Simulation}

The governing equations comprise a set of coupled, nonlinear partial differential equations and algebraic constraints to impose conservation of mass, momentum, energy and the evolution of individual species mass. For the application presented here, the low Mach number approximation is applied. This problem was formulated in terms of primitive variables - pressure, velocity, temperature, and species mass fractions. The details of these equations are presented in the previous chapter.

In this work, GRI-Mech 3.0 [74] is used within CHEMKIN [64] subroutines to model combustion without nitrogen chemistry. This mechanism includes 36 species and 219 reactions.

Since a uniform grid was applied in developing this model, in order to have a grid independent solution, the whole domain $(6 \mathrm{~cm})$ was first divided into 300 control volumes $(\Delta r=$ $0.02 \mathrm{~cm})$. In the second simulation, the number of control volumes was doubled to $600(\Delta r=$ $0.01 \mathrm{~cm})$ and eventually increased to $1200(\Delta r=0.005 \mathrm{~cm})$. These three simulations were used to compare the solutions' differences by calculating GCI (Grid Convergence Index) [75]. In order to calculate GCI, the following Eq. (3.1) was used:

$$
G C I_{\text {fine }}^{21}=\frac{1.25 e_{a}^{21}}{r_{21}^{p}-1}
$$

where $r_{21}$ is the ratio of the control volume size in the first and second simulations $\left(r_{21}=2\right), p$ and $e_{a}^{21}$ are defined in Eq. (3.2) and Eq. (3.3), respectively. 


$$
p=\frac{1}{\ln \left(r_{21}\right)}|\ln | \frac{\varepsilon_{32}}{\varepsilon_{21}}|+q(p)|
$$

where $\varepsilon_{32}=\epsilon_{3}-\epsilon_{2}, \varepsilon_{21}=\epsilon_{2}-\epsilon_{1}, \epsilon$ is the solution on the corresponding grid, and $q(p)=0$ for constant $r$.

$$
e_{a}^{21}=\left|\frac{\epsilon_{1}-\epsilon_{2}}{\epsilon_{1}}\right|
$$

The value of GCI calculated for the fine grid was an order of magnitude less than $1 \%$ (typical acceptable range in CFD is $1 \%-5 \%$ ). In order to ensure that minor species, such as $\mathrm{H}, \mathrm{OH}$, and $\mathrm{CH}$ (which have sharp gradients compared to other species in the flame), are resolved in the grids used in the simulation, their differences in one control volume on various grids were studied. Minor species concentrations changed by less than $5 \%$ between neighbouring control volumes with the fine grid (600 control volumes).

In terms of time steps used in transient computations, according to [37], the choice of $\Delta t=$ $\theta / 200$ where $\theta$ is the period of oscillations, was found to be generally sufficient. Therefore, selecting constant $\Delta t=10^{-06} \mathrm{~s}$ is reasonable for the simulations up to $2000 \mathrm{~Hz}$. A simple analysis of reaction time scales shows that the typical evolution time for the species is of the order of $1.1 \times 10^{-05} \mathrm{~s}$, which is several orders of magnitude smaller than the smallest oscillation flow time scale $\left(\tau_{\text {flow }}=0.0005 \mathrm{~s}\right)$ for the perturbations that travels through the preheat and reaction layers. Therefore, selecting a constant time step of $10^{-06} \mathrm{~s}$ is adequate for capturing all the chemical and flow computations. 


\subsubsection{Solution Methodology}

A standard solution approach for such flow problems is to use staggered grids in the context of finite-volume methods [66]. Therefore, a finite volume formulation was derived for the onedimensional total mass, momentum, energy, and species equations. The diffusive terms were discretized using a second-order central difference scheme while the convective terms were discretized using the exact exponential solution to approximate the values between the centers of two control volumes [67]. The boundary conditions were based on the underlying physical characteristics of the model. For the inlet boundary (burner surface): $\dot{m}=\dot{m}_{0}+B \sin (\omega t)$, specified mass flow (for steady state $\mathrm{B}=0$ ), $d p / d r=0$, zero gradient for pressure, $T=T_{u}$ (unburned mixture temperature), $Y_{k}=Y_{k, u}$ (unburned mixture conditions) and for the outlet boundary conditions: $d \dot{m} / d r=0$, zero gradient for mass flow rate $(d(\rho u A) / d r=0), p=p_{a t m}$, atmospheric pressure, $d T / d r=0$, zero gradient for temperature, $d Y_{k} / d r=0$, zero gradient for species mass fractions were applied, assuming that the boundary was far enough from the flame that the flow composition is no longer appreciably changing.

Once the coupled, nonlinear system of equations had been discretized, the system was solved iteratively using Newton's method at each time step and continues until the maximum norm of the solution vector was reduced to within a specified tolerance. Since the convergence of Newton's method requires a sufficiently good initial guess and many convergence problems with this method can be eliminated by applying time-stepping, a time-dependent approach is used to solve this one-dimensional flame problem instead of solving a steady-state boundary value problem directly. This method has been chosen mainly because it is robust and it converges for sufficiently small time steps. Newton's method has been used to solve the system of nonlinear governing equations for each time step. Typically, the relative (scaled) tolerance should be in the 
range of $10^{-3}$ to $10^{-4}$ [8]. The linear system formed in Newton iteration was solved using a $\mathrm{Bi}$ CGSTAB linear solver [70]. In general, the Bi-CGSTAB convergence criteria $\left(10^{-07}\right)$ should be a few order of magnitudes smaller than Newton's method convergence tolerance $\left(10^{-04}\right)$.

Since the problem was solved with dimensional variables, as opposed to their nondimensional counterparts, it is necessary to discuss the actual physical size of the computational domain. Consequently, the calculated results could be expected to depend on the radius of curvature of the flame if it were of the same order of magnitude as the flame thickness. To avoid these size dependencies, the inflow boundary was set to be $50 \mathrm{~mm}$ such that the radius of curvature of the flame $(80 \mathrm{~mm})$ was at least 20 times that of its thickness $(3 \mathrm{~mm})$.

\subsection{Results and Discussion}

\subsubsection{Steady State Condition}

For ignition, the reactant mixture at $300 \mathrm{~K}$ was introduced into pure nitrogen at $1800 \mathrm{~K}$, which initially filled the computational domain. The temperature and species fields evolve until they reach steady state. It is worth pointing out that this stationary flame was solved by time stepping of the transient model with steady boundary conditions. Figure 3.1 depicts the species mass fractions and temperature profile of the stationary flame at the stoichiometric condition. To ensure the final product condition was met, the solution was tested for domain length independence by doubling its size and verifying solution consistency. In order to achieve a mesh independent solution and also ensure that the resolution is sufficient to capture the thin reaction layers of minor species, a non-uniform grid was used with spacings varying from $0.02 \mathrm{~cm}$ to $0.005 \mathrm{~cm}$. This

stationary flame was used as the initial condition of the time-dependent boundary condition flame 
and also to compare some of its characteristics with expanding and contracting flames at various frequencies.

(a)

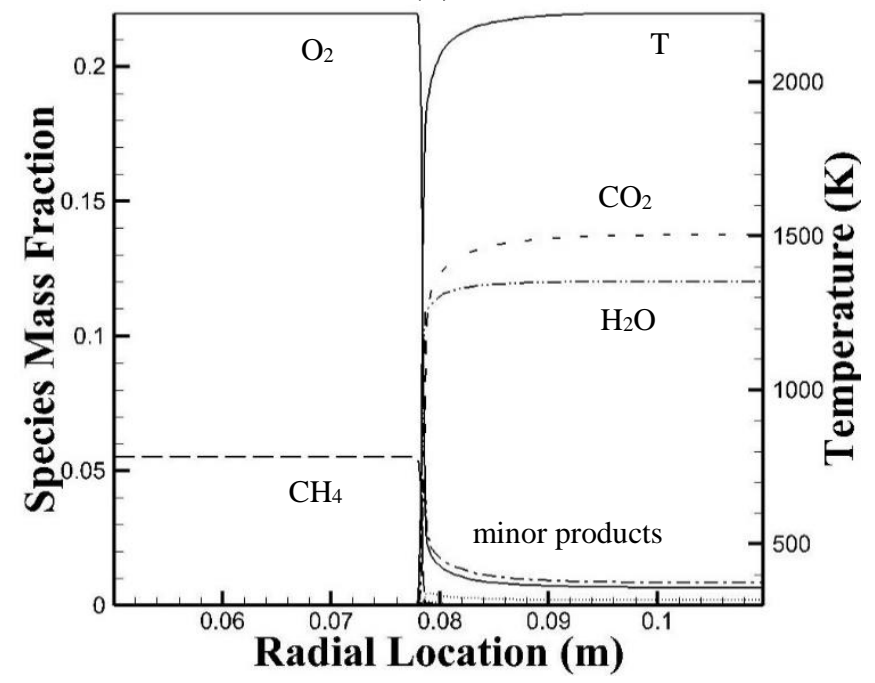

(b)
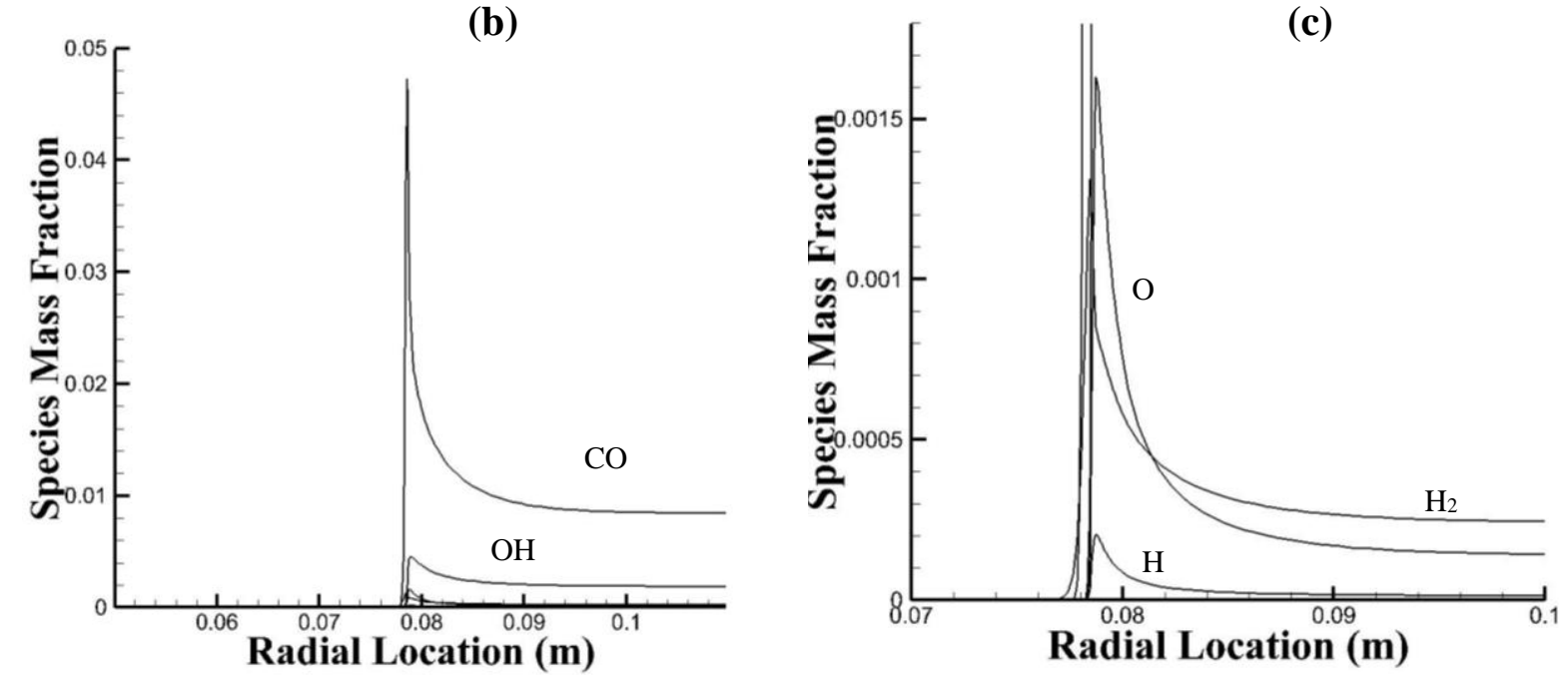

Fig. 3.1 (a) Temperature and mass fractions of major species, (b) and (c) Species mass fractions of minor adiabatic product species

Prior to presenting the periodic results, steady computational results were verified against appropriate conditions in the literature. Currently, there are no experimental results for this exact 
geometry, so the results were compared to those of others in terms of the flame velocity, temperature, and species mass fractions. The unstretched structure of steady flame in this geometry and the small ratio the flame radius to its thickness make this comparison valid. The first test was a comparison with the equilibrium state temperature and composition. The Chemical Equilibrium Calculation Spreadsheet [76] which invokes JANAF thermodynamical tables [77] was used to estimate the equilibrium state. In Table 3.1, the equilibrium results are compared to the burned gas composition calculated herein at the end of the computational domain where all gradients were zero, and showed very good agreement.

Table 3. 1: Comparison of the equilibrium state and burned gas of major species and some minor species

Equilibrium State Burned Gas

\begin{tabular}{ccc}
\hline Temperature (K) & 2213.3 & 2213.6 \\
\hline \multicolumn{3}{c}{ Major Species Mass Fractions } \\
\hline $\mathbf{C H}_{4}$ & 0.00000 & 0.00000 \\
\hline $\mathbf{O}_{2}$ & 0.00616 & 0.00615 \\
$\mathbf{N}_{2}$ & 0.72500 & 0.72500 \\
\hline $\mathbf{H}_{2}$ & 0.00002 & 0.00002 \\
\hline $\mathbf{H}$ & 0.000001 & 0.000001 \\
\hline $\mathbf{O}$ & 0.00001 & 0.00001 \\
\hline $\mathbf{O H}$ & 0.00182 & 0.00175 \\
\hline $\mathbf{H}_{2} \mathbf{O}$ & 0.12043 & 0.12042 \\
\hline $\mathbf{C O}$ & 0.00855 & 0.00801 \\
\hline $\mathbf{C O}$ & 0.13838 & 0.13829 \\
\hline
\end{tabular}

As discussed previously, even though this stationary flame has a finite curvature, it is not stretched. To compare to planar unstretched flames it is important to avoid the potential impact of 
this curvature. The flame radius of curvature was $\sim 80 \mathrm{~mm}$, while the flame thickness was $\sim 3.2 \mathrm{~mm}$ so this flame can be considered sufficiently flat to warrant comparison to a planar premixed flame. Therefore, similar to a one-dimensional planar laminar premixed flame with stoichiometric methane-air reactants, the flame speed should be close to $36.6 \mathrm{~cm} / \mathrm{s}$ in the stoichiometric flame ( $\varphi=1.0)$ and $28.6 \mathrm{~cm} / \mathrm{s}$ in the fuel lean flame $(\varphi=0.8)$ [78]. The flow velocity in the domain near the flame is shown in Fig. 3.2, where a decrease in unburned gas mixture velocity can be observed in the vicinity of $r=0.08 \mathrm{~m}$ due to the outward flow around the cylinder. Once the flow enters the flame region, the velocity reaches a minimum of $36.6 \mathrm{~cm} / \mathrm{s}$ for the stoichiometric flame, $28.6 \mathrm{~cm} / \mathrm{s}$ for the fuel lean flame $(\varphi=0.8)$ and $17.5 \mathrm{~cm} / \mathrm{s}$ for $\varphi=0.7$. Also shown in Fig. 3.2 as horizontal dashed lines, are the estimated planar unstretched laminar burning velocities of a stoichiometric and a fuel lean methane-air flame at atmospheric pressure reported in [78] which has been selected here for comparison as it is among the more classical studies in flame velocity measurements. There is excellent agreement between the experiments and the current simulation, demonstrating that the steady state solution computed here captures the basic physical processes of a methane-air flame. 


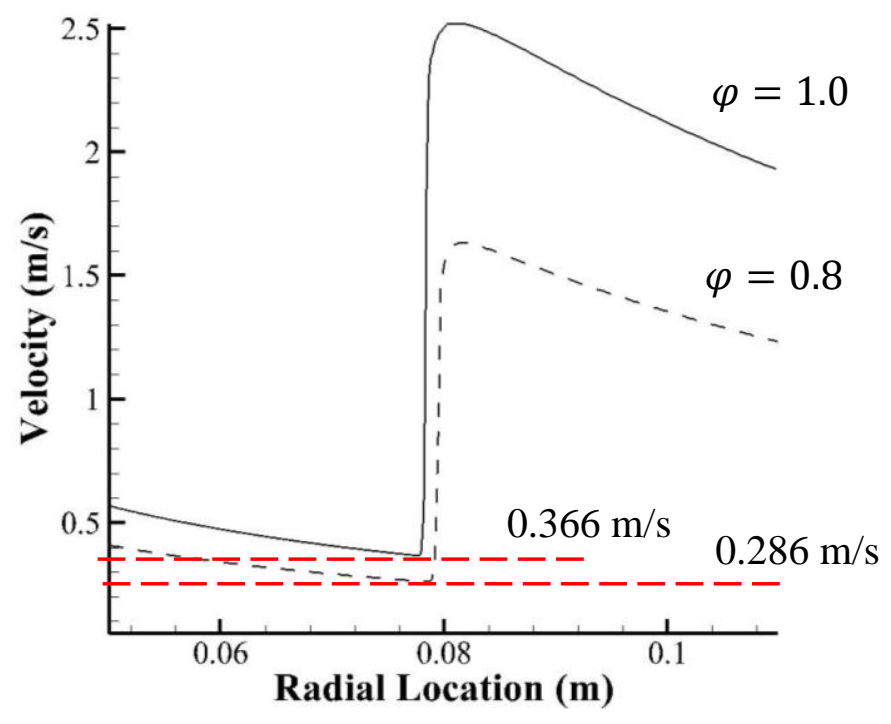

Fig. 3.2 The velocity profile of a stationary flame for comparison with flame speeds for the planar flame geometry given in [78]

As mentioned previously, the steady state solution was obtained based on the transient code with steady boundary conditions. Therefore, another technique to validate the transient time stepping of the code is to compare the ignition delay time obtained by the code to the values reported in the literature based on experimental results. According to [79], ignition delay time of a methane-air flame can be calculated using the following equation:

$$
\tau=1.09 \times 10^{-3} p^{-0.68} \varphi^{-0.04} \exp \left(\frac{40.98}{R T}\right)
$$

where $\tau$ is ignition delay time in $\mu s, p$ is pressure in atm, $\varphi$ is equivalence ratio, $R=1.986 \times$ $10^{-03} \mathrm{kcal} /(\mathrm{mol} \mathrm{K})$ is the universal gas constant, and $T$ is temperature in $\mathrm{K}$. This equation was used to calculate the ignition delay time for three different flames as follows:

$$
p=1 \mathrm{~atm}, \mathrm{~T}=1800 \mathrm{~K}, \varphi=1.0,0.8 \text {, and } 0.7
$$


The calculated ignition delay times based on Eq. (3.4) and the values obtained by the simulations were in the order of $100 \mu s$ with only a few micro seconds difference. For instance, for equivalence ratio of 0.8 , the calculated ignition delay time is equal to $104 \mu s$ and the simulated value is $110 \mu \mathrm{s}$. Therefore, in order to validate the transient code; first, the time stepping of the transient governing equations were used to reach the steady state solution. In the next step, the steady state solution was compared in terms of flame burning rate and the equilibrium state of all the dependent variables. To ensure that the time stepping to reach the steady flame was sufficiently accurate, the ignition delay time of the flame was compared against the experimental results. With regard to the fact that the flame ignition delay time was captured by the computational model, the code has obtained the steady solution through an accurate time dependent path.

\subsubsection{Transient Periodic Flow}

For transient flow cases, the inlet boundary condition of mass flow per unit axial length changes sinusoidally, given by:

$$
\dot{m}^{\prime}=\dot{m}_{0}^{\prime}+B \sin (\omega t)
$$

In this equation, $B$ was set equal to $10 \%$ of $\dot{m}_{0}^{\prime}\left(\dot{m}_{0}^{\prime}=0.2\right) \mathrm{kg} / \mathrm{m} . \mathrm{s}$ and represents the amplitude of the oscillation and $\omega$ is the frequency, which is varied between $5 \mathrm{~Hz}$ and $2000 \mathrm{~Hz}$. This lower limit was needed because the effects of unsteadiness for low equivalence ratio mixtures were observed even at these conditions. The reason to increase the frequency up to $2000 \mathrm{~Hz}$ is for the relevance to turbulent flows. For example, in the middle of a premixed turbulent duct flame stabilized by a backward facing step, mean frequency of the flame motion across a fixed point was 
observed to be in this range [80]. Experiments show that the wrinkling process of flamelets depends on the geometry of turbulent flame [81]. Therefore, it has been suggested to study premixed turbulent flames in different geometries (i.e., envelope flames, oblique flames, unattached flat flames, and spherical flames) separately. In order to study all aspects of flamelets, a wide range of amplitude, frequency and curvature should be analyzed. However, as the first step, in this chapter, the amplitude and curvature have been kept constant and frequency and equivalence ratio have been considered the manipulating variables. As mentioned previously, in general, turbulent flames can freely move away from high values of stretch rate, which is an absent component in restrained flames in the counter-flow configuration. It is worth noting that this model is capable of analyzing a wide range of stretch rate by increasing the amplitude of modulation and decreasing the radius of curvature.

The dynamics of premixed flames have been frequently studied by monitoring the flame displacement speed, which suffers from having arbitrarily defined a datum within the scalar structure of the flame [82]. Similarly, to quantify the stretch rate experienced by a flame of finite thickness, a datum (i.e., $F=0$ in Eq. (1.2)) needs to be specified. This same problem exists here as the mass flux varies with radial position across the flame. Selecting an iso-surface as the flame surface is arbitrary and results presented become dependent on that choice. To overcome this challenge for now, an integral quantity calculated over the whole domain, independent of the domain size, will be used to compare the stationary flame with the dynamics of a curved flame at different frequencies. This quantity is the chemical energy source term integrated for all species and in all control volumes in the domain, and defined as: 


$$
E I^{\prime}=\sum_{i=1}^{N_{c v}} \sum_{k=1}^{N_{s p}} h_{k} \dot{\omega}_{k} \mathbb{V}^{\prime}(i)
$$

where $E I^{\prime}$ is referred to here as the energy index (per unit axial length), and $\mathbb{V}^{\prime}(i)$ represents the volume of cell $i$ (per unit axial length). Also, by comparing this quantity at the same point in consecutive cycles, it was used to determine if the solution had reached the fully-periodic state from its initial conditions.

In order to have a better comparison between different equivalence ratios, the flame speeds $\left(v_{f}\right)$ and the steady values of $E I^{\prime}$ at the mean mass flow rate (steady state case which has been used as the reference conditions) are summarized in Table 3.2:

Table 3.2: The flame speed and steady state $E I^{\prime}$ at the mean mass flow rate

\begin{tabular}{lccc} 
& $\boldsymbol{\varphi}=\mathbf{1 . 0}$ & $\boldsymbol{\varphi}=\mathbf{0 . 8}$ & $\boldsymbol{\varphi}=\mathbf{0 . 7}$ \\
\hline $\boldsymbol{v}_{\boldsymbol{f}}(\mathbf{c m} / \mathbf{s})$ & 36.6 & 28.6 & 17.5 \\
\hline$E I^{\prime}(\mathbf{J} / \mathbf{s})$ & 5264325 & 3215720 & 1955668 \\
\hline
\end{tabular}

After reaching steady state for each equivalence ratio, the solution was used as the initial condition for the transient cases. With regard to this exact steady solution, the transient code solves the time-dependent governing equations using Newton's method at each time step. After a number of initial cycles, a fully developed solution was obtained for each frequency. To reach a fullyperiodic state, the code runs for multiple cycles and the variations in successive cycles were plotted to determine if the results were fully periodic and suitable for analysis. It was expected that as the number of cycles increases, the flow approaches a fully periodic and repeating state. To track the 
convergence to the fully-periodic state, a normalized correlation function $(C F)$ [15] was used to calculate the variations in the sum of $E I^{\prime}$ at two successive cycles $(j)$ :

$$
C F(j)=\frac{\sum_{t=(j-1) / f}^{t=(j / f)} E I^{\prime}(j, t)-\sum_{t=(j-2) / f}^{t=(j-1) / f} E I^{\prime}(j-1, t)}{\sum_{t=(1 / f)}^{t=(2 / f)} E I^{\prime}(2, t)-\sum_{t=0}^{t=(1 / f)} E I^{\prime}(1, t)}
$$

where $f$ is the frequency. As illustrated in Fig. 3.3, each data set decays to negligible variation after 9-12 cycles. Based upon these results, the comparisons have been made using the computational data in the tenth cycle.

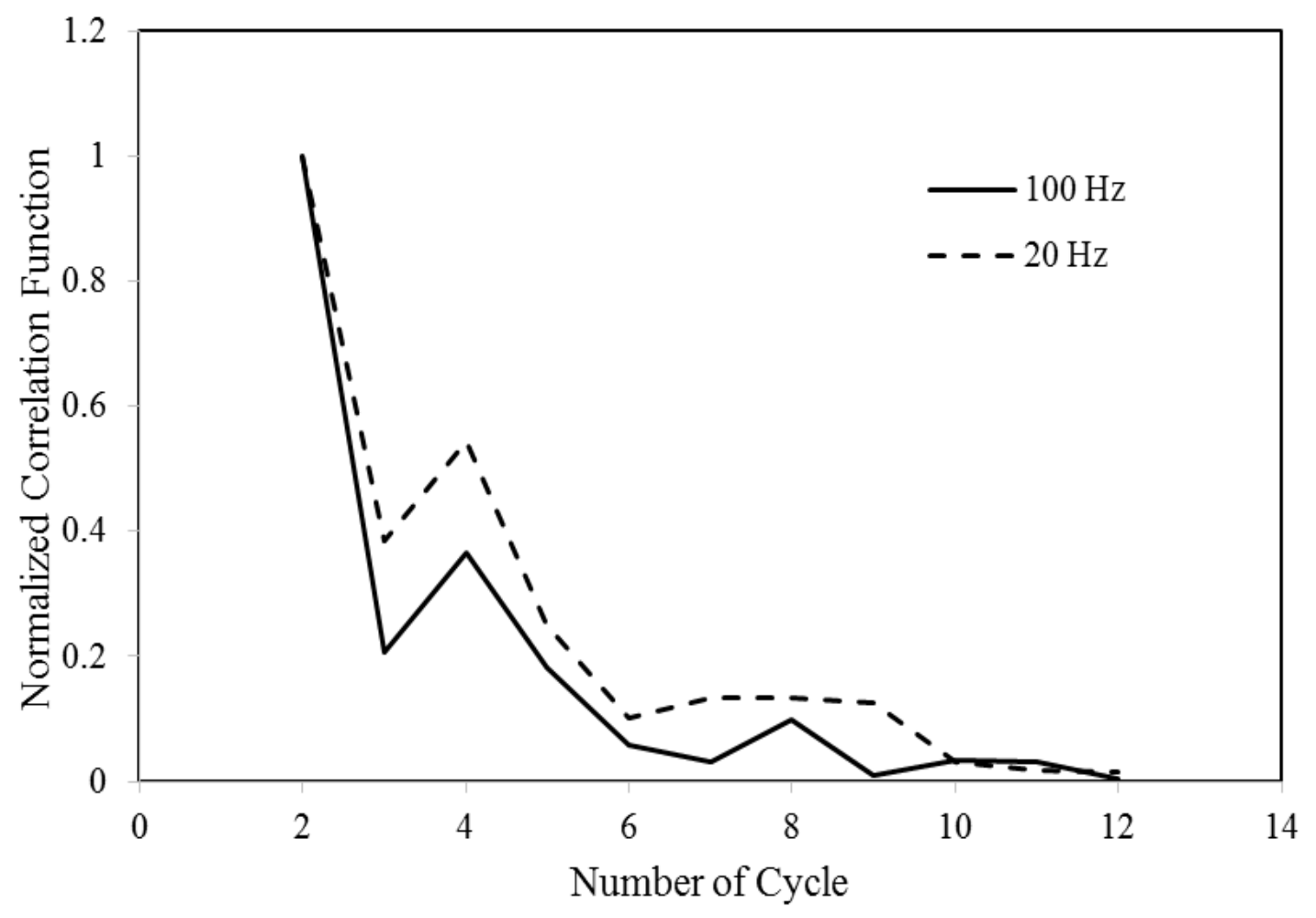

Fig. 3.3 Normalized correlation function $(C F(j))$ versus cycle number $(j)$ for stoichiometric condition 
In Table 3.3, EI' has been calculated and compared for different conditions; namely, steady state, expanding, and contracting flames, when the value of instantaneous mass flow rate was equal to that of the steady state ( $E I^{\prime}$ has the units J/s per unit axial length, but has been normalized here by the steady state value). Since $E I^{\prime}$ is an integral value of heat release due to chemical reaction over the whole domain, it is related to the amount of mass entering the flame area. It has been discussed previously that due to the difficulty and ambiguity of defining a leading flame surface to relate $E I^{\prime}$ to flame radius, the frequency response of the flame has been plotted versus the mass flow rate, and comparisons have been made using these integral values at the same mass flow rate, rather than at the same flame radius.

Table 3.3: Comparison of the normalized energy index $\langle E I\rangle$ of expanding and contracting flames at different frequencies

\begin{tabular}{c|cc|cc}
\hline \multirow{2}{*}{ Frequency } & \multicolumn{2}{|c|}{$\phi=\mathbf{1 . 0}$} & \multicolumn{2}{c}{$\boldsymbol{\phi}=\mathbf{0 . 8}$} \\
\cline { 2 - 5 } & $\begin{array}{c}\text { Expanding } \\
\text { Flame }\end{array}$ & $\begin{array}{c}\text { Contracting } \\
\text { Flame }\end{array}$ & $\begin{array}{c}\text { Expanding } \\
\text { Flame }\end{array}$ & $\begin{array}{c}\text { Contracting } \\
\text { Flame }\end{array}$ \\
\hline $\mathbf{2 0 ~ H z}$ & 0.994 & 1.006 & 0.990 & 1.010 \\
\hline $\mathbf{2 0 0 ~ H z}$ & 0.975 & 1.025 & 0.966 & 1.034 \\
\hline $\mathbf{2 0 0 0 ~ H z}$ & 0.980 & 1.020 & 0.985 & 1.015 \\
\hline
\end{tabular}

At each frequency, the contracting flame has a value higher than unity and the expanding flame has a value lower than unity. This result is expected since the expanding flame has a positive stretch and the contracting flame has a negative stretch. The essential point being that at these instants for the two separate equivalence ratios, their imposed inlet upstream hydrodynamic state is identical, but extra information is required regarding whether this instant is part of an expanding, contracting, or stationary flame in order to accurately estimate the instantaneous rate of energy 
conversion. While the differences between stationary, expanding, and contracting at this instant appear to be modest (+/- 1 to $3 \%$ for these frequencies, which is linked to the relatively small fluctuations in mass flow rate), it is interesting to note that this characteristic is not monotonic with frequency for either equivalence ratio. Therefore, it is worth following the flame response throughout a complete cycle. The small variations observed in Table 3.3 are mainly due to two causes. First, the methane-air flame with Le 1 (Lewis number (Le) is a dimensionless number defined as the ratio of thermal diffusivity to mass diffusivity) is generally stretch insensitive suggesting that the transient flame responses should not deviate substantially from the instantaneous response. It should be noted that this mixture has been selected to keep consistency with similar works in other configurations. Second, in order to stay relatively close to the linear response limit, the amplitude change of mass flow rate is kept small at $+/-10 \%$ throughout this study.

Figure 3.4-a shows how $E I^{\prime}$ changes at different frequencies of mass flow rate oscillation as a function of the phase of the mass flow (i.e., the phase is zero when the flow rate is at its mean value and increasing) compared to the flame response based on the quasi-steady assumption for an equivalence ratio of unity. With the quasi-steady assumption (solid line), changing the mass flow rate sinusoidally with time at the inlet affects the value of $E I^{\prime}$ in the flame. Therefore, the flame responds to the change in mass flow rate instantaneously with no phase lag or amplitude change. In this regard, each point on the solid line corresponds to its own steady radius. As can be seen in Fig. 3.4-a, the amplitude of the oscillations of $E I^{\prime}$ diminish and its phase shift increases with an increase in frequency to as much as $\sim \pi / 4$ at $2000 \mathrm{~Hz}$. Although the results of premixed methane-air flames can be compared qualitatively to those of the counter-flow configuration, the significant distinguishing feature of this study compared to previous works is the unstrained nature 
of the flame in the new geometry. Therefore, the present work helps to fill the knowledge gap of transient response considering the effects of changing curvature and strain rate separately. Different levels of hydrogen addition could result in more deviations in flame transient response due to its smaller Lewis number.

In Fig. 3.4-b, $E I^{\prime}$ has been plotted with respect to the inlet mass flow rate as it varies throughout a cycle for stoichiometric conditions for the quasi-steady and transient response for different frequencies (from $20 \mathrm{~Hz}$ to $2000 \mathrm{~Hz}$ ) as indicated. The straight line in the middle of the closed pathways indicates the flame response to the periodic variation in mass flow rate based on the quasi-steady assumption. In the transient response, as the frequency rises, the straight line transforms into an elliptical shape (the path in this phase-space is counter-clockwise) with an incline toward horizontal. Figure 3.4-b allows for comparison of the $E I^{\prime}$ at the same mass flow rate (along a single vertical line) but when the flame is either expanding (lower portion of the ellipse) or contracting (upper portion of the ellipse), and emphasizes the deviation from the quasi-steady model. 

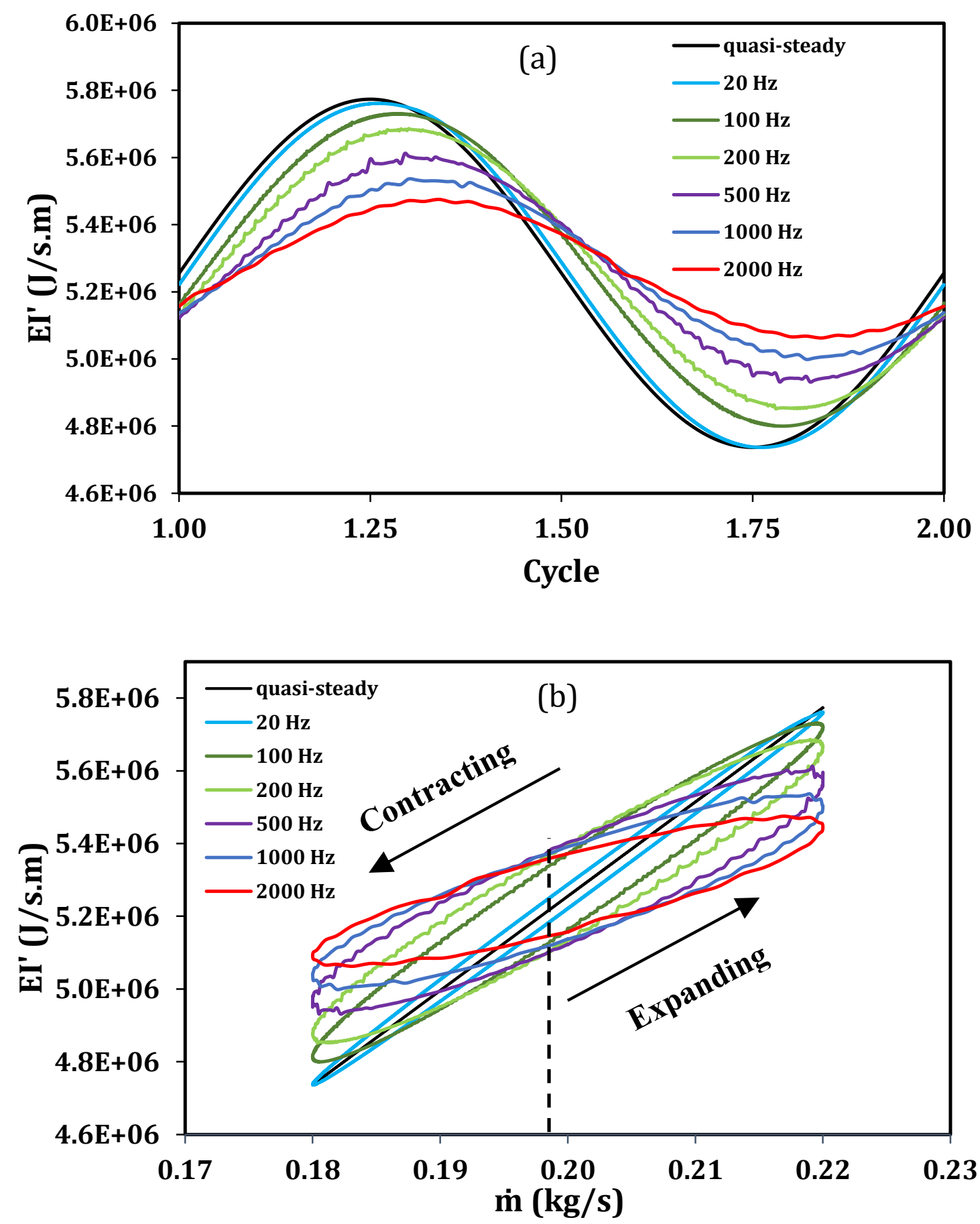

Fig. 3.4 a) Periodic change in $E I^{\prime}$ in one cycle for quasi-steady and transient response to frequencies from $20 \mathrm{~Hz}$ to $2000 \mathrm{~Hz}$, b) $E I^{\prime}$ versus mass flow rate for quasi-steady and transient response to frequencies from $20 \mathrm{~Hz}$ to $2000 \mathrm{~Hz}$ 
In Fig. 3.4-b, as the frequency increases, the difference between $E I^{\prime}$ values of expanding and contracting flames at the mean mass flow rate first increases and then decreases, represented by the initial widening then contracting of the ellipses with increasing frequency.

Figure 3.5-a, shows how EI' amplitude (i.e., difference between maximum and minimum values of $E I^{\prime}$ at each frequency) decreases monotonically with an increase in frequency for stoichiometric and fuel lean flames. For an equivalence ratio of 0.7 this amplitude is reduced by a factor of $\sim 5$ at a frequency of $2000 \mathrm{~Hz}$, which essentially decouples burning rate from the changes in the stretch rate, while for the stoichiometric flame the reduction is only by a factor of $\sim 2.5$. In Fig. 3.5-b, a comparison of $E I^{\prime}$ at the instant where the mass flow rate equals the mean has been made between the stoichiometric condition $(\varphi=1.0)$ and two fuel-lean conditions $(\varphi=0.8$ and $\varphi=0.7)$. The maximum difference between the expanding and contracting flames' $E I^{\prime}$ in the stoichiometric flame occurs at higher frequency compared to in the fuel lean flames. Taken collectively, Fig. 3.5 shows that as the reactant mixture deviates further from stoichiometric conditions and towards extinction that the flames' responses appear to become more sensitive to the dynamics of stretch. 

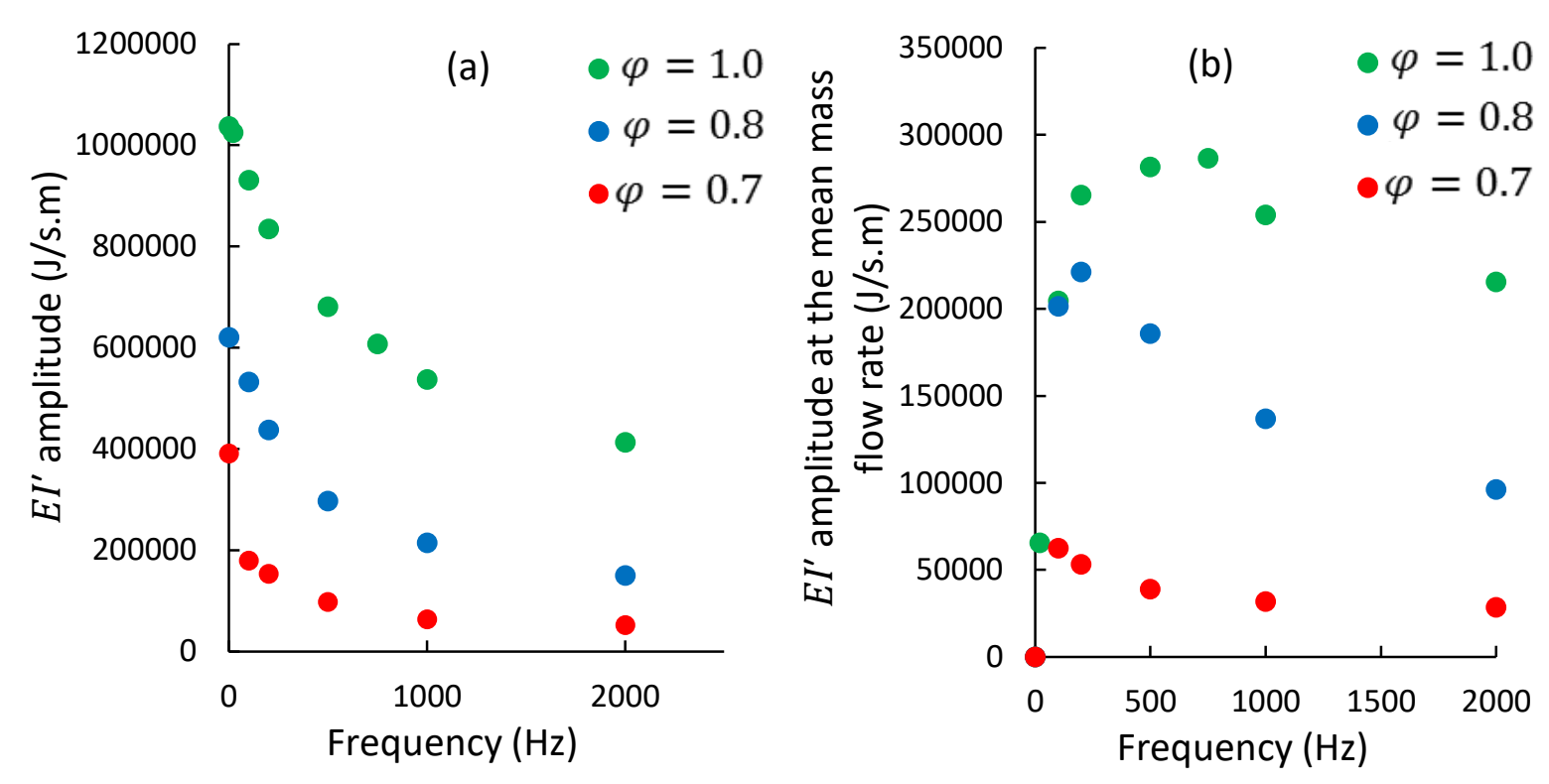

Fig. 3.5 a) The difference between maximum and minimum values of $E I^{\prime}$ ( $E I^{\prime}$ amplitude) vs. frequency for stoichiometric and fuel lean flames, b) Difference between $E I^{\prime}$ values of expanding and contracting flame at the mean mass flow rate vs. frequency for stoichiometric and fuel lean flames

For a better understanding of the effect of equivalence ratio on the transient response of laminar premixed flames to a periodic variation in mass flow rate over a wide range of frequency, $\langle E I\rangle$ which is defined by the ratio of $E I^{\prime}$ to $E I^{\prime}{ }_{s S}$ (i.e., $E I^{\prime}$ at steady state for the mean mass flow rate), has been plotted versus $\langle\dot{m}\rangle$ (the mass flow rate normalized the by the mean mass flow rate) at each frequency for stoichiometric $(\varphi=1.0)$ and two fuel lean $(\varphi=0.8$ and $\varphi=0.7)$ flames in Fig. 3.6. In this regard, each point on the transient curves and quasi-steady lines is normalized against one single mean value of $E I^{\prime}{ }_{s S}$ for that specific equivalence ratio. 

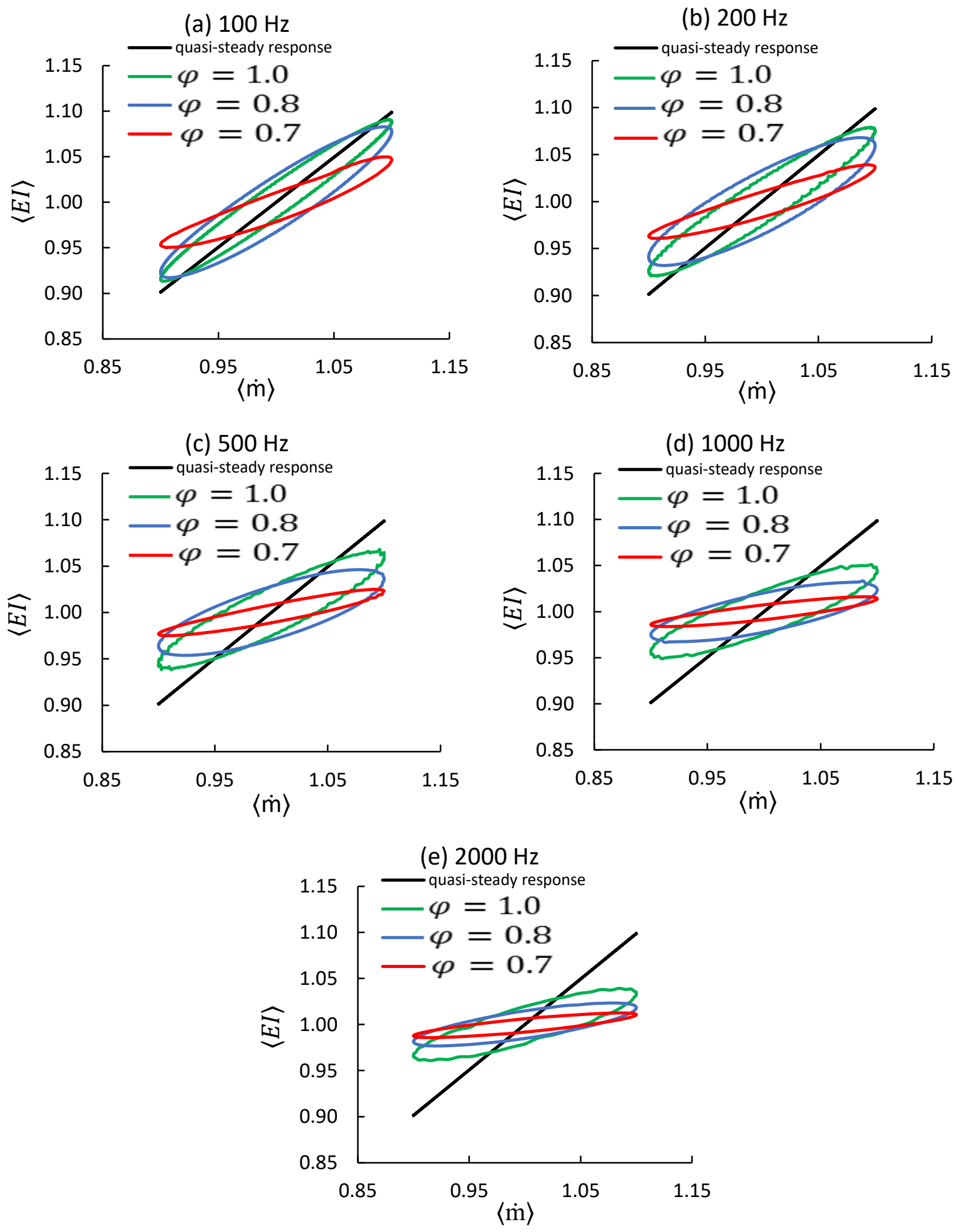

Fig. 3.6 The effect of equivalence ratio on transient response of laminar premixed flame to various frequencies. Normalized $E I^{\prime}$ (by $E I^{\prime}$ of steady state flames) vs. normalized mass flow rate at (a) $100 \mathrm{~Hz}$, (b) $200 \mathrm{~Hz}$, (c) $500 \mathrm{~Hz}$, (d) $1000 \mathrm{~Hz}$, and (e) $2000 \mathrm{~Hz}$ for stoichiometric $(\varphi=$ $1.0)$ and two fuel lean $(\varphi=0.8$ and $\varphi=0.7)$ flames 
To ensure that the responses presented in Fig. 3.6 are not affected by any other factors such as oscillating about different mean radial locations, the mass flow rates have been adjusted so that the flames with three different equivalence ratios stabilize at the same position. Otherwise, with the same mean mass flow rate, the leaner flames would be farther away from the inflow and it would show a smaller stretch amplitude locally, and consequently the response of $E I$ amplitude would decrease. The comparison between a stoichiometric flame and fuel lean flame with equivalence ratio of 0.8 for various frequencies from $100 \mathrm{~Hz}$ to $2000 \mathrm{~Hz}$ indicates that the elliptical shapes in the fuel lean flame inclined more toward the horizontal line with a smaller major axis at all frequencies and larger minor axis at lower frequencies (less than or equal to $500 \mathrm{~Hz}$ ) and smaller minor axis at higher frequencies (greater than or equal to $1000 \mathrm{~Hz}$ ). Therefore, as the equivalence ratio decreases to 0.7 , a considerable change can be observed in the transient response of the flame, which means that fuel lean flames are more sensitive to changes in frequency compared to stoichiometric flames.

\subsubsection{Time Scale Analysis of Flame Response}

In order to have a better understanding of the transient responses presented in the previous section, the results were studied from the perspective of time scales, because one of the main differences attributed to changing equivalence ratio is the flame speed and related quantity of the chemical time scale. In this regard, the Damköhler number was selected as the indicator of the interaction between the flow field and chemistry. Due to the different responses of the flames to periodic flows, there is not a unique time scale for either the flow or chemistry, and therefore there could be different definitions of the Damköhler number. One definition of Damköhler number could be the ratio of imposed flow time scale to unstretched steady state chemical time scale: 


$$
D a_{1}=\frac{(1 / f)}{\alpha /\left(S_{L}^{0}\right)^{2}}=\frac{\left(S_{L}^{0}\right)^{2}}{\alpha f}
$$

In this equation, $f$ is the frequency of the mass flow rate at the inlet boundary, $\alpha$ is the thermal diffusivity and $S_{L}^{0}$ is the unstretched laminar flame velocity. This definition has the advantage of being evaluated on quantities that are specified (imposed) on the flow, or known $a$ priori, such as the material properties. The Damköhler number could also be defined by considering the flame's response to the changing hydrodynamics. In the previous section, with increasing frequency it was observed that $E I^{\prime}$ had a diminishing amplitude and increasing phase lag, which aligns with observations of diminishing flame movement and increasing phase lag [3, $34,7]$, meaning that flame response may be important to consider when comparing flames of different equivalence ratios. Essentially, variations in the flow were imposed on the flame, but the stretch rate experienced by the flames in terms of their changing positions and thermo-chemical structures are responding variables. Therefore, a second Damköhler number is also considered for which the flow time scale is based on the variation in stretch rate that includes the frequency and flame response, though not explicitly altering the chemical time scale due to varying chemical structures in the flame.

$$
D a_{2}=\frac{(1 / \kappa)}{\alpha /\left(S_{L}^{0}\right)^{2}}=\frac{\left(S_{L}^{0}\right)^{2}}{\alpha \kappa}
$$

To calculate a characteristic stretch rate (with stretch rate amplitude defined as the difference between maximum and minimum stretch rate in a cycle) in each case, Eq. (1.1) was 
used. As mentioned, due to the particular geometry of the flame, the stretch rate equation is simplified to $\kappa=\left\{v_{f} \nabla \cdot \boldsymbol{n}\right\}_{F=0}$. Considering flame stretch rate, the flame displacement speed is used to analyze the dynamics of premixed flames. This quantity is generally ambiguous except for with a steadily propagating planar flame. The main reason is that the mass flow rate through the combustion region varies with distance through the flame. This variation makes it difficult to select an iso-surface to represent the flame surface. In the present work, as $v_{f}$ represents local flame speed, selecting an iso-surface is challenging. Therefore, this quantity is sensitive to iso-surface selection. In order to apply the simplified stretch rate equation to a flame, an arbitrary isotherm needs to be selected where the velocity and divergent to the normal of the flame front is calculated. As suggested in [82], an iso-surface close to the hot side of the flame is less sensitive to the magnitude of the calculated stretch rate. Therefore, the selected isotherm is where temperature rise reaches $90 \%$ of the total temperature increase. Fig. 3.7 illustrates how the radius of this isotherm and its corresponding stretch rate change with time in an oscillating flame with equivalence ratio of 0.8 and frequency of $20 \mathrm{~Hz}$. Comparing the two plots, there are two locations where flame stretch rate becomes zero (where the flame stops moving and changes direction); maximum and minimum stretch rates occur when the flame is expanding and contracting while passing through the mid-point (mean mass flow rate), respectively. 


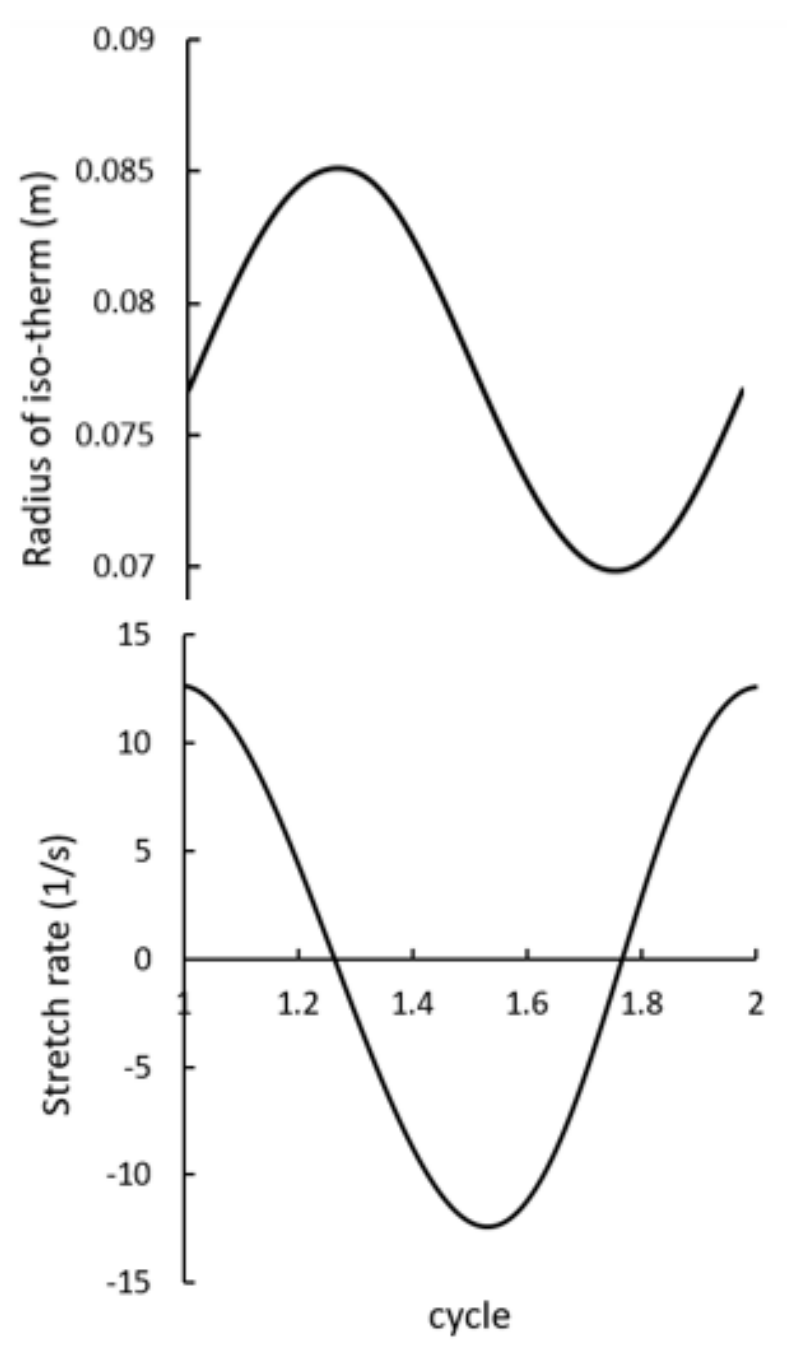

Fig. 3.7 Changes in the radius of the isotherm (top) and stretch rate (bottom) in a cycle for equivalence ratio of 0.8 and frequency of $20 \mathrm{~Hz}$

Figure 3.8 depicts the stretch rate amplitudes that characterize the flame responses for the different equivalence ratios over the range of frequencies tested, and shows the connection between the two time scales. As expected for a constant equivalence ratio, as the frequency rises, the stretch rate amplitude increases due to the higher time rate of change of the iso-surface radius, though not linearly because of the lagging response of the flames. Also, at the same frequency, lower equivalence ratio flames have smaller stretch rate amplitude. The main reason is that the 
oscillating flame amplitude is smaller in lower equivalence ratios due to the greater phase lag discussed earlier. Therefore, the amplitude of the iso-surface becomes smaller with a constant rate of change of radius.

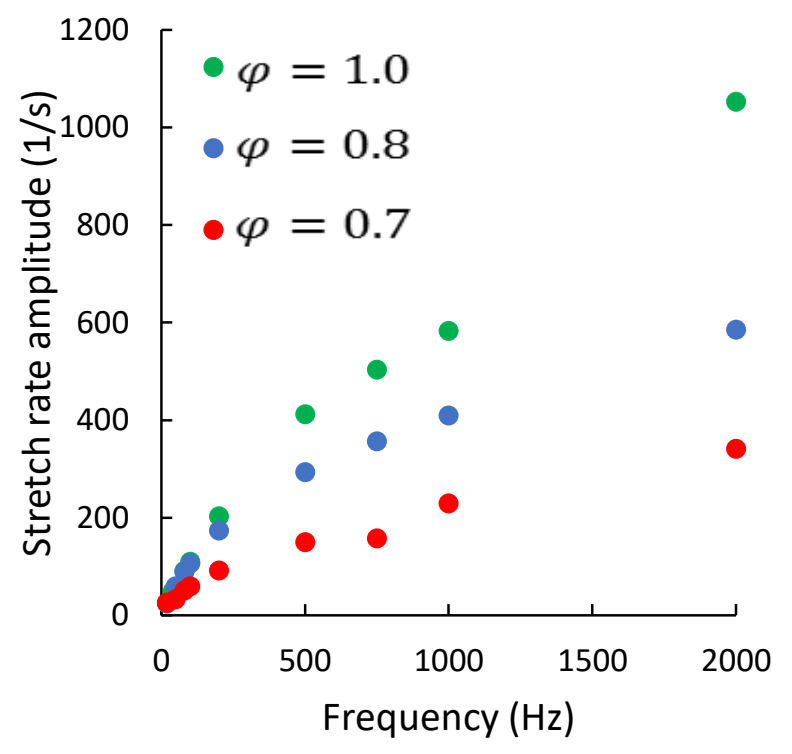

Fig. 3.8 The changes of stretch rate amplitudes vs. frequency for three different equivalence ratios

Fig. 3.9 illustrates the normalized $E I$ amplitude at the mean mass flow rate with respect to the two different definitions of Damköhler number for an equivalence ratio of 0.8. In this figure, the flow time scale is calculated based on frequency (solid line) and stretch rate (dashed line). At lower frequencies the Damköhler Number based on the stretch rate is smaller than that based on the frequency, while the opposite is true for the higher frequencies when the flame cannot respond to such rapid changes. The characteristic stretch rate considered in $D a_{2}$ calculations is defined as the difference between maximum and minimum stretch rates (stretch rate amplitude). As illustrated in Fig. 3.8, the stretch rate amplitude increases with frequency at each equivalence ratio in the same order of magnitude. This increase suggests that at the limit of very high frequencies, the 
stretch rate amplitude is also increased to a range that makes $D a_{2}$ very small (close to zero). Due to the similarity of the two plots, the characteristic Damköhler Number based on imposed frequency was used in the remainder of this chapter.

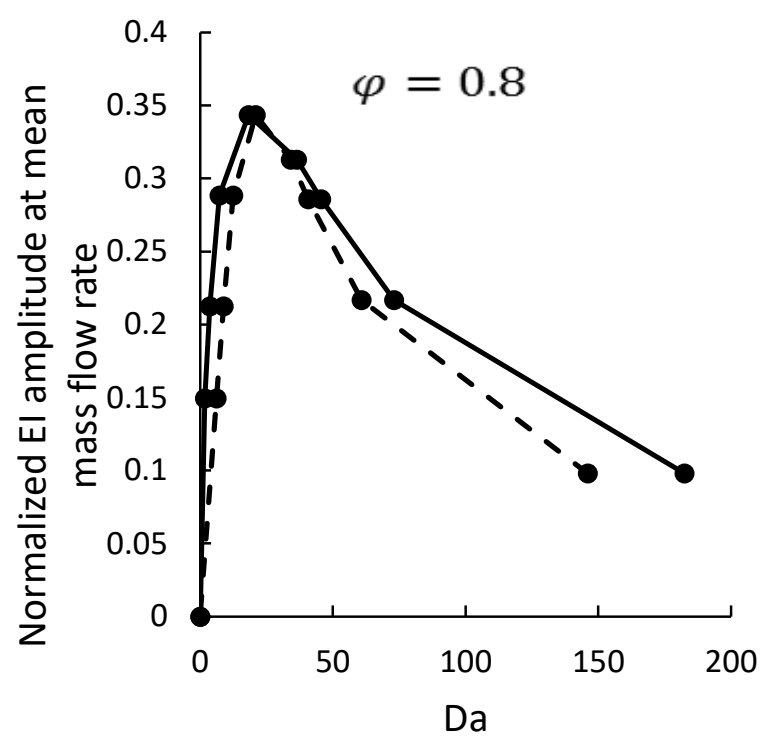

Fig. 3.9 Normalized EI amplitude at mean mass flow rate with respect to the Damköhler number calculated based on Eq. (3.8) (solid line) and Damköhler number calculated based on Eq. (3.9) (dashed line) for equivalence ratio of 0.8 .

Figure 3.10 shows how the normalized EI amplitude varies with Damköhler number. For each of these equivalence ratios the curves appear S-shaped, approaching unity at high Damköhler Numbers (low frequencies). Interestingly, the effects of unsteady stretch on the rate of energy conversion can be observed even when the time scale of the flow variations are two orders of magnitude lower that the flames' chemical time scales, especially for the equivalence ratio of 0.7 . While the stoichiometric case and that with equivalence ratio of 0.8 appear to be well correlated by the Damköhler Number, especially at high Damköhler Numbers, the response of the equivalence ratio of 0.7 suggests that the Damköhler Number does not alone capture the effects of 
unsteady stretch. Also, in this figure, the rate of change of $E I$ amplitude is more pronounced in lower Damköhler numbers and it is less sensitive to frequency in higher Damköhler numbers. This sensitivity suggests a change in flame response to periodic flow when the flow time scale approaches the chemical time scale. This change in the slopes can be related to the maximum point in Fig. 3.11.

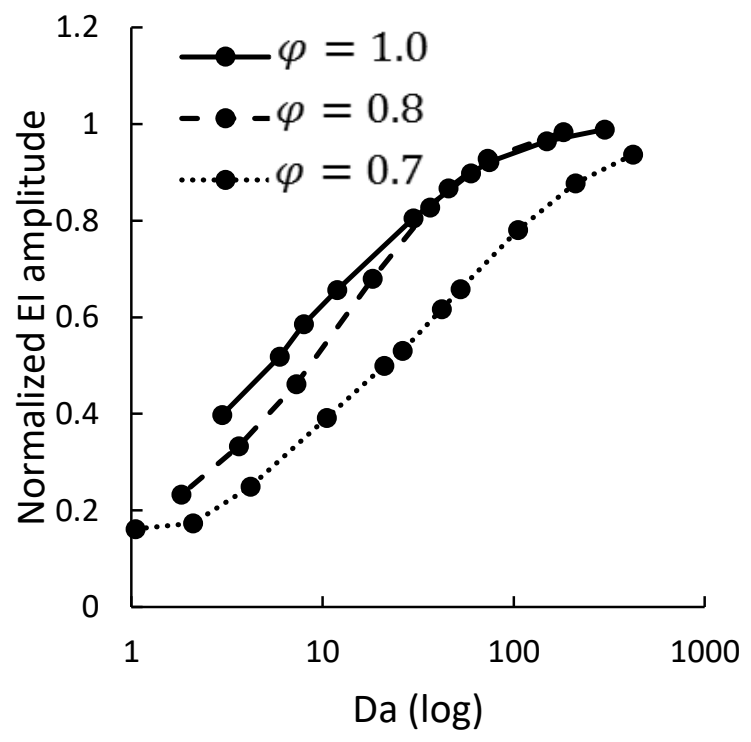

Fig. 3.10 Normalized EI amplitude with respect to Damköhler number $\left(D a_{1}\right)$ for three equivalence ratios

Figure 3.11 shows the normalized EI amplitude at the mean mass flow rate (i.e., minor axis of ellipses in Fig. 3.6), plotted with respect to Damköhler number (Eq. 3.4). As can be observed, there is a maximum point in all three plots. As the equivalence ratio decreases, these maxima move to higher Damköhler numbers (i.e., lower frequencies). 


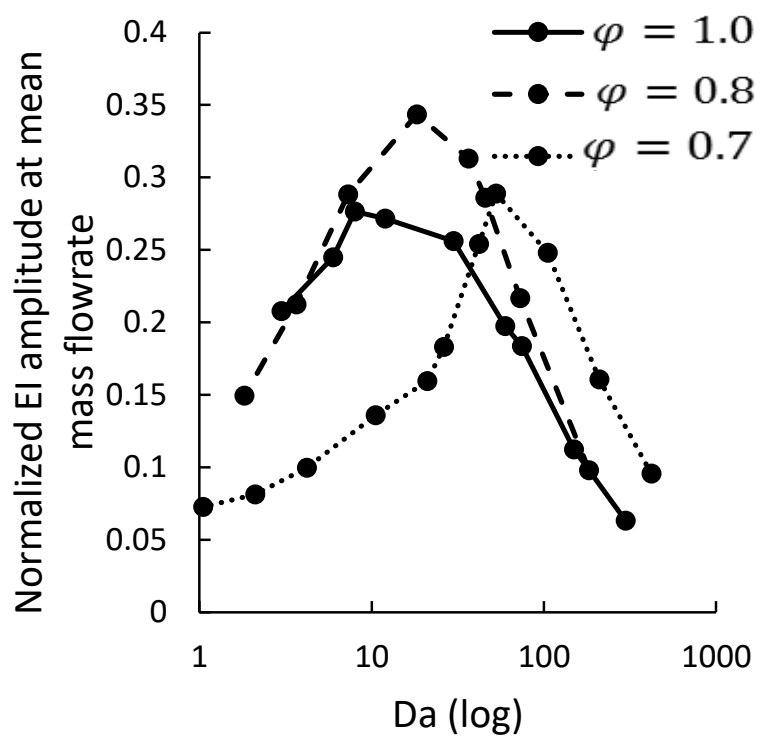

Fig. 3.11 Normalized EI amplitude at mean mass flow rate vs. Damköhler number for three equivalence ratios.

In this figure, $E I^{\prime}$ amplitude at the mean mass flow rate is the difference between maximum and minimum values of $E I^{\prime}$ at each frequency, which is then normalized by the $E I^{\prime}$ amplitude in the quasi-steady condition. The responding behaviour of the different equivalence ratio flames is not well captured by the Damköhler Number, with the data for the equivalence ratio of 0.7 being dramatically different from that of the other two cases.

\subsection{Summary}

A model was developed in order to revisit the quasi-steady assumptions that are used in current approaches of premixed laminar flamelet models. This model is based on a cylindricallysymmetric radial outward flow geometry with an applied sinusoidal variation of mass flow rate that was only $10 \%$ of the mean flow rate. The flame response was studied when the flow and scalar fields align (i.e., no tangential strain), while the flame either expands (positive stretch) or contracts (negative stretch). This transient model was validated against the burning velocity, temperature, 
and species mass fractions of a stationary flame. Since the stationary flame in this geometry is not stretched but has a finite curvature, an investigation was made to compare key characteristics of steady unstretched flames to the dynamics of a stretched flame due to the motion of the curved flame front. The parameter used for this comparison was the total chemical enthalpy to thermal enthalpy conversion across the flame, $E I^{\prime}$.

It has been concluded that similar to planar flames exposed to oscillating strain rates for which the flame stretch always has a positive value, the transient response of laminar premixed flames results in decreasing amplitudes of motion and burning rates, and increasing phase lag with increasing frequency. This changing $E I^{\prime}$ amplitude and phase lag resulted in an elliptical shape when it was plotted with respect to the instantaneous mass flow rate, which showed the different burning rates between positively and negatively stretched flames with the same imposed hydrodynamic state, as well as how that differed from the quasi-steady state.

A comparison was also made between stoichiometric and two fuel lean flames to study the effect of equivalence ratio on the transient response of laminar premixed flames to various frequencies. It was concluded that as the equivalence ratio was made leaner, so that the chemical time scales became longer, the flame's burning rate became more susceptible to lower frequency variations in the mass flow. To try to correlate this behavior to flow variations the data was reanalyzed with two different Damköhler numbers (one based on a flow time associated with the imposed frequency, and the other based on a flow time associated with the fluctuations in stretch rate). While all the flames examined showed similar characteristics with respect to either Damköhler Number, no quantitative correlations in unsteady burning rate quantities were observed. An important observation was that the unsteadiness in burning rate was seen at flow times that were two orders of magnitude slower than the flame's chemical time scales. 
The results presented here have implications for premixed turbulent combustion modeling using the laminar flamelet approach. In premixed turbulent combustion, curved flamelets are surrounded and convected by time-varying vortices that stretch the flame. Flamelet libraries described by steady planar strained flames in the counter-flow configuration can be challenged by this instantaneous structure of the turbulent flame, because the local flame front includes positive stretch and negative stretch. The model that has been proposed and developed in this thesis indicates how expanding (positive stretch), contracting (negative stretch), and steady flames (unstretched) can behave differently at various frequencies and equivalence ratios in terms of chemical energy release. This phenomenon stresses the fact that in order to generate a more rigorous flamelet library to model a premixed turbulent flame, the time-dependent motion of a curved flame in both positive and negative stretch rates should be considered. 


\section{Chapter 4: Using Frequency Response Analysis to Predict Unsteady Burning Rate from Unsteady Stretch Rate}

This chapter is based on the following published paper:

M. Sahafzadeh, S. B. Dworkin, L. W. Kostiuk, "Using frequency response analysis to predict unsteady burning rate from unsteady stretch rate," Submitted to Combustion and Flame (under review), July 2017.

\subsection{Introduction}

Linear and nonlinear flame transfer functions or flame describing functions have been widely used to study the flame instability in turbulent combustion. In these functions, the input is considered to be velocity fluctuations and output is usually heat release in chemical reactions. Although the mathematical approach is similar to those of instability analysis, in this study, the application of these functions is to capture the transient effects of stretch rate due to the motion of curved flames. This information can be implemented in turbulent combustion modeling which modifies flamelet library to define the properties of turbulent flames more accurately.

In order to apply flame dynamics to flamelet library, one needs to know the flame response to unsteady stretch at each specific condition. However, due to the complexities in laminar flame modeling which includes detailed chemistry, simulation of each case is not efficient; therefore, a simpler method is required to capture these effects. The scope of the current work comprises an investigation of a flame transfer function to predict the transient response of a curved laminar premixed flame to inlet oscillations in mass flow rate.

The frequency response analysis was applied to this study, to map the flame behavior in the form of a simple closed mathematical relation. The other advantage of using transfer functions 
is that they are independent of the input excitations; therefore, the system response can be estimated for any arbitrary inputs such as step functions, ramp functions, or unsteadiness of turbulence.

\subsection{Frequency Response Analysis}

The transient responses that have been studied in this chapter were generated in the manner similar to that in previous work [83], in which a laminar premixed flame was simulated in a cylindrically-symmetric geometry (Fig. 2.1). Details of the cylindrically-symmetric flame model are omitted here for concision. For a complete model description, the reader is referred to [83].

The mass flow rate was changed sinusoidally with time (Eq. 3.5) at the inlet boundary. Eq. (3.5) was applied as the inlet boundary condition in the simulations), and could be seen as the input to the transfer function. It should be noted that this changing mass flow rate or inlet flow velocity leads to stretch rate variations due to the outward and inward motion of the curved flame. Therefore, an alternate and more useful variable to understanding the unsteady response of flames is to consider stretch rate as the input. This approach is beneficial in that it can be used in modifying the steady flamelet library for unsteady situations. This approach is discussed in the following section in more detail.

In Eq. $3.2, \dot{m}$ the mass flow rate at the inlet boundary, $\dot{m}_{0}$ the mean mass flow rate in steady state condition, $B$ is equal to $10 \%$ of $\dot{m}_{0}$ and represents the amplitude of the oscillation, and $\omega$ is the angular frequency. The frequency was varied between $20 \mathrm{~Hz}$ and $2000 \mathrm{~Hz}$. As mentioned previously, lean flames have become the focus of recent research due to their applicability to lowemission combustion systems. Thus, in this study, the equivalence ratio was varied from 1.0 to 0.7 in order to compare the stoichiometric condition to lean flames. The reason to increase the frequency up to $2000 \mathrm{~Hz}$ is for the relevance to turbulent flows. For example, in the middle of a 
premixed turbulent duct flame stabilized by a backward facing step, mean frequency of the flame motion across a fixed point was observed to be in this range [80].

The geometry in Fig. 2.1 represents unique features of flame dynamics compared to the counter-flow configuration, which is usually used to generate flamelet libraries. The steady flame is unstretched due to the balance of curvature and normal strain. However, when the flame is exposed to a flow field fluctuation, the expanding flame is subjected to positive stretch and the contracting flame generates negative stretch, which has been ignored in the literature. The magnitude of stretch rate could be changed by varying mean flame curvature, amplitude of oscillations, frequency of oscillations, equivalence ratio, and fuel type.

The dynamics of premixed flames has been frequently characterized by the flame displacement speed (FDS), which often suffers from being arbitrarily defined. A similar situation exists here that the mass flux varies with radial position. Selecting an iso-surface as the flame surface is arbitrary and any results presented become tied to that choice. To overcome this challenge, an integral quantity over the whole domain, independent of the domain size, has been used to compare the stationary flame with the dynamics of a curved flame at different frequency. This quantity is the chemical energy source term integrated for all species and in all control volumes in the domain, and is defined by Eq. 3.3. This energy released during the combustion process was calculated, and was considered the output of the system.

In Eq. 3.3, $E I^{\prime}$ is referred to here as the energy index, and $\mathbb{V}(i)$ represents the volume of cell $i$ and $H_{k}$ is the enthalpy of species $k$. Two global characteristics of the system (amplitude attenuation and phase angle change) can be observed in Fig. 4.1. The solid line in all figures illustrates the quasi-steady response of the flame to a change in inlet mass flow rate. In the quasisteady condition, the flame responds to the inlet mass flow rate instantaneously regardless of the 
equivalence ratio and the frequency of the oscillation. However, the transient response depends on the equivalence ratio and the frequency of the perturbations. As can be seen at each frequency, the flame response is more delayed with decreasing equivalence ratio. In this condition, the amplitude of the flame response is also damped at lower equivalence ratios. At constant equivalence ratio, increasing the frequency has a similar influence on the flame response. Therefore, the phase lag increases and the amplitude decreases with an increase in frequency. 

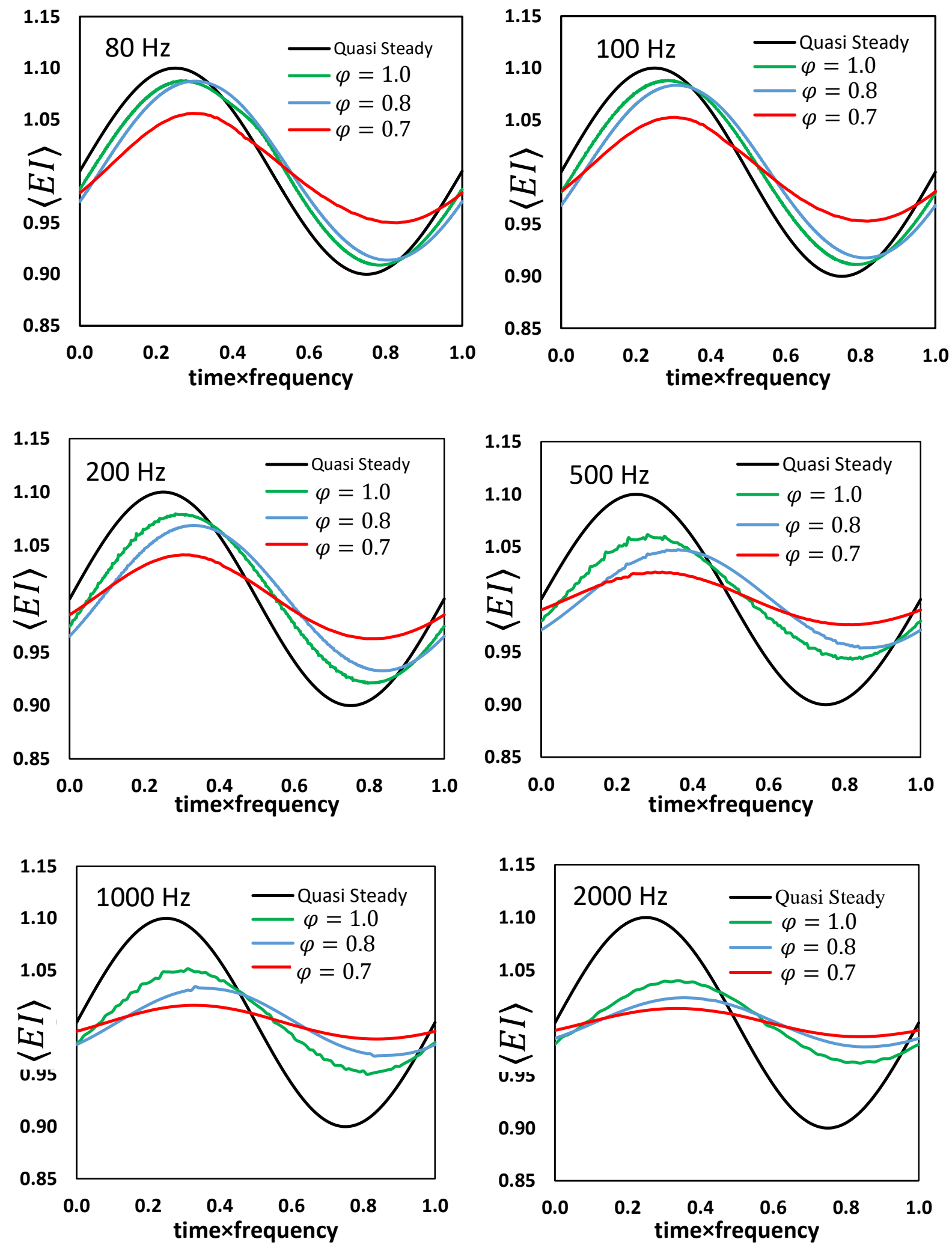

Fig. 4.1 Quasi steady and transient responses of flames with equivalence ratios of $\varphi=1.0, \varphi=$ 0.8 and $\varphi=0.7$ at a range of frequencies from $80 \mathrm{~Hz}$ to $2000 \mathrm{~Hz}$. 
In Fig. 4.2, a similar analysis was performed to study the effect of mean flame curvature. The original simulations in Fig. 4.1 were done with mean flame curvature of $8 \mathrm{~cm}$. As can be seen in Fig. 4.2, when the mean curvature was halved to $4 \mathrm{~cm}$, the transient results were not changed significantly compare to the sensitivities seen in Fig. 4.1.
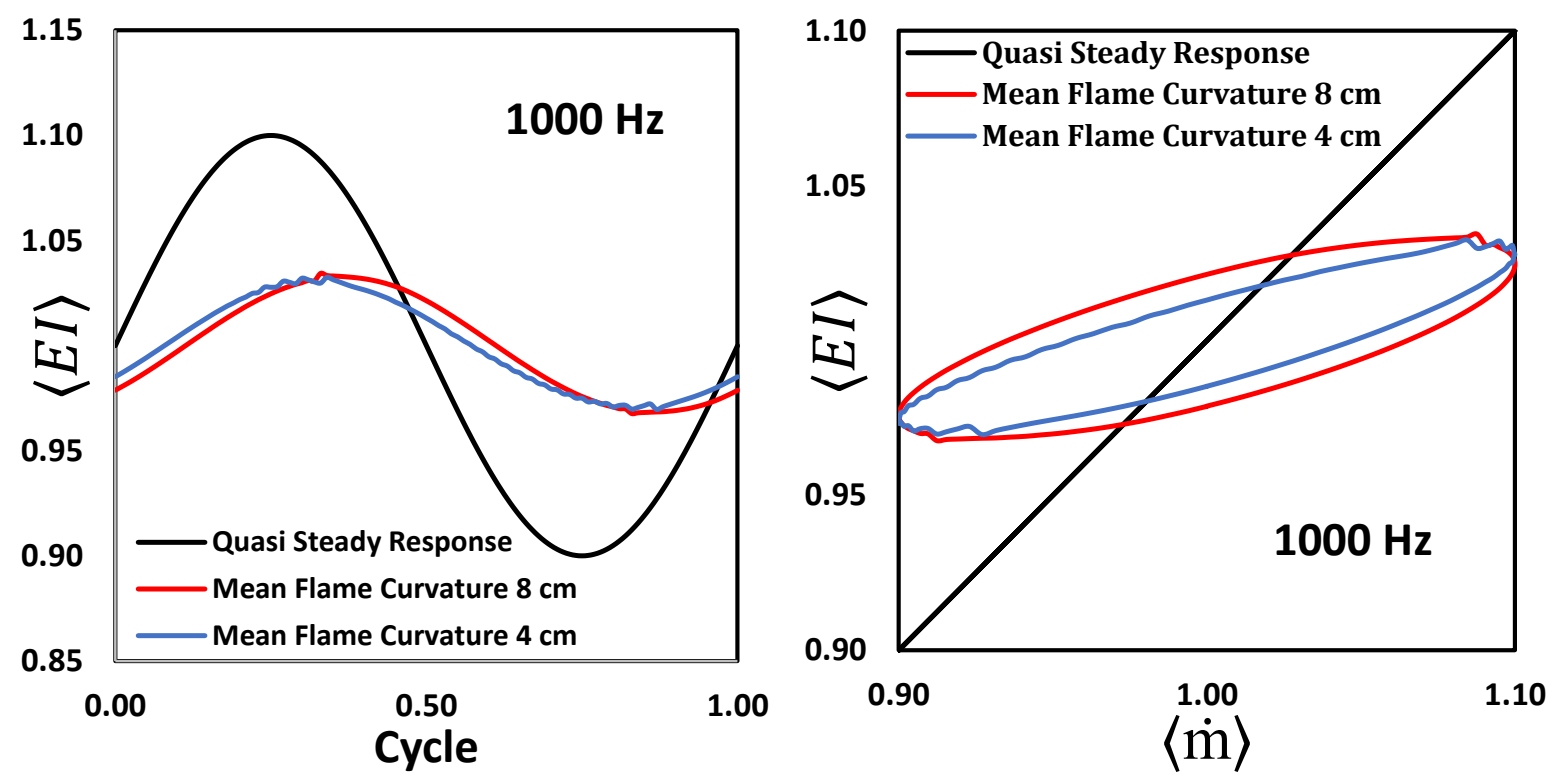

Fig. 4.2 Quasi-steady and transient responses of flames with two mean flame curvatures; left side: normalized $E I^{\prime}$ in one cycle, right side: normalized $E I^{\prime}$ vs. normalized mass flow rate (equivalence ratio $\varphi=0.8$ and frequency $1000 \mathrm{~Hz}$ ).

From this analysis, it is obvious that the flame response to a change in upstream mass flow rate depends primarily on parameters such as equivalence ratio and the frequency of oscillations. Therefore, it would seem desirable to find a describing function to replace the cumbersome complex chemistry computations of transient response of turbulent and laminar premixed flames. It should be noted that this analysis is aimed at characterizing the response of the flame to perturbations. Due to the complexity of a flame with detailed chemistry and several species 
involved in the reactions, understanding the physical mechanism that created the response is not the focus of this study.

In this chapter, the capabilities of different models have been studied in order to estimate the transient responses in terms of $E I^{\prime}$, and these predicted values were then compared to those of full complex chemical numerical results. The models chosen for this analysis include treating the system as linear first order, higher order linear, and a specific type of non-linear system. It is worth mentioning that this analysis only includes one relatively small value of amplitude (10\% of the steady state case) in the reactant mass flow rate.

As $E I^{\prime}$ is an integral value which combines flame speed and flame surface area effects, an analysis was required in order to separate these two competing effects. Figure (4.3) shows the instantaneous consumption speed as a phase plot with respect to the corresponding instantaneous stretch rate. The quasi-steady response is a point (red dot in middle of the figure) located on $\kappa=0$ and $S_{L}=0.26$, which is essentially the steady state value and will be referred to as $S_{L}^{O}$. At very low frequencies $(20 \mathrm{~Hz})$, the relationship between local flame speed and stretch rate is almost linear. By further increasing the frequency, the line becomes an ellipse. The slope of the ellipses are negative which has been shown in [81] for a methane-air flame at the same range of equivalence ratio. The sensitivity of the local flame speed to the stretch rate first increases in the low frequency regime, and then decreases in the high frequency regime. 


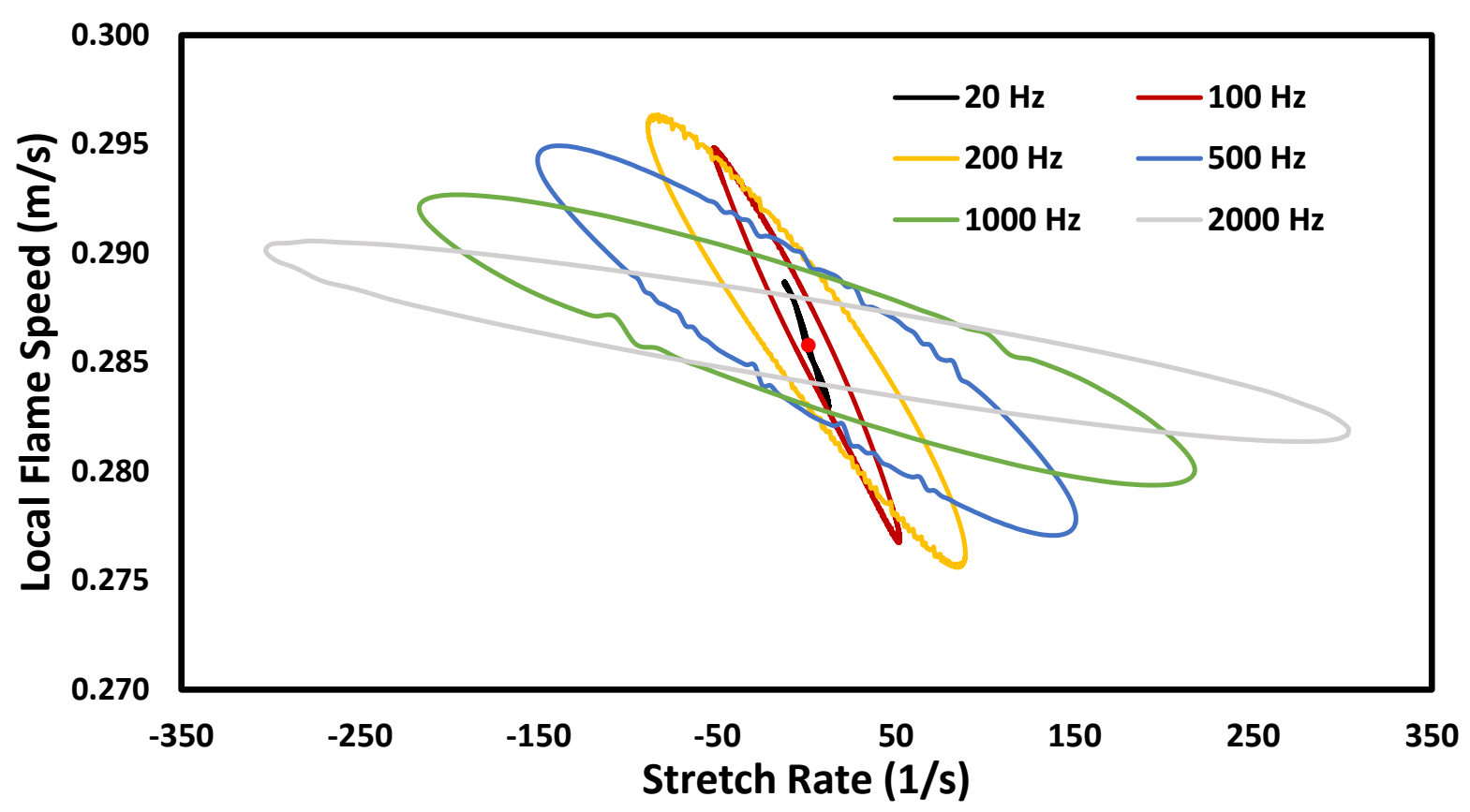

Fig. 4.3. Local flame speed vs. stretch rate for equivalence ratio of 0.8 and frequencies between $20 \mathrm{~Hz}$ and $2000 \mathrm{~Hz}$

\subsubsection{Linear First Order System Analysis}

In this section, a first order linear model is used in order to analyze the flame behavior at each equivalence ratio. Equation 4.1 indicates the relation between different parameters of a first order linear system.

$$
\frac{B}{A}=\frac{k}{\sqrt{\tau^{2} \omega^{2}+1}}
$$

In this equation, $\boldsymbol{B}$ is the amplitude of the output, $\boldsymbol{A}$ is the amplitude of the input, $\boldsymbol{k}$ is the gain of the system, $\boldsymbol{\tau}$ is the time constant, and $\boldsymbol{\omega}$ is the frequency of oscillations. For our purposes here $\boldsymbol{B}$ is $\dot{E} I$, while $\boldsymbol{A}$ is $\dot{m}(t)$ multiplied by the specific enthalpy of reaction to convert the reactants to the equilibrium product state to be the flow of chemical enthalpy. In order to illustrate the ratio 
of amplitudes, $\frac{B}{\boldsymbol{A}}$ in Eq. 4.1, consider the quasi-steady response of the flame to a periodic change in mass flow rate. The ratio of output to input is just $\boldsymbol{k}$, and the gain of the system becomes unity. As illustrated in Fig. 4.4-(a), a linear first order model has been fitted to the numerical results for an equivalence ratio of 1.0 over the whole range of frequencies. Reasonable agreement can be observed for the low range of frequencies. As defined in [83], in this context, low-frequency is the range that chemistry time scale is smaller than flow time scale (inverse of frequency of oscillation) and depending on the equivalence ratio (flame burning velocity). Figure 4.4-(b) indicates that good accuracy of predicting the results using a first order linear approximation is due to the flame behavior in the low frequency range. The same approach in Fig. 4.4-(d) and 4.4-(e) shows similar results for an equivalence ratio of 0.7 , though for a significantly smaller frequency range. Therefore, with relatively good accuracy, a first order linear system can be used for this flame at low frequencies.

To improve the model, the power in the denominator in Eq. 4.1 has been changed to account for a nonlinearity parameter ( $\xi)$. (Eq. 4.2)

$$
\frac{B}{A}=\frac{k}{\left(\tau^{2} \omega^{2}+1\right)^{\xi}}
$$

Figures 4.4-(c) and 4.4-(f) illustrate the potential of nonlinear models to accurately predict transient responses for equivalence ratios of 1.0 and 0.7 , respectively. While this curve fit shows good agreement over the whole range of frequencies, the nonlinear transfer functions cannot be estimated with the current approach because the inverse Laplace transform of a nonlinear function 
that is needed to get the transfer function is not possible and thus an alternate methodology is used in the next section.
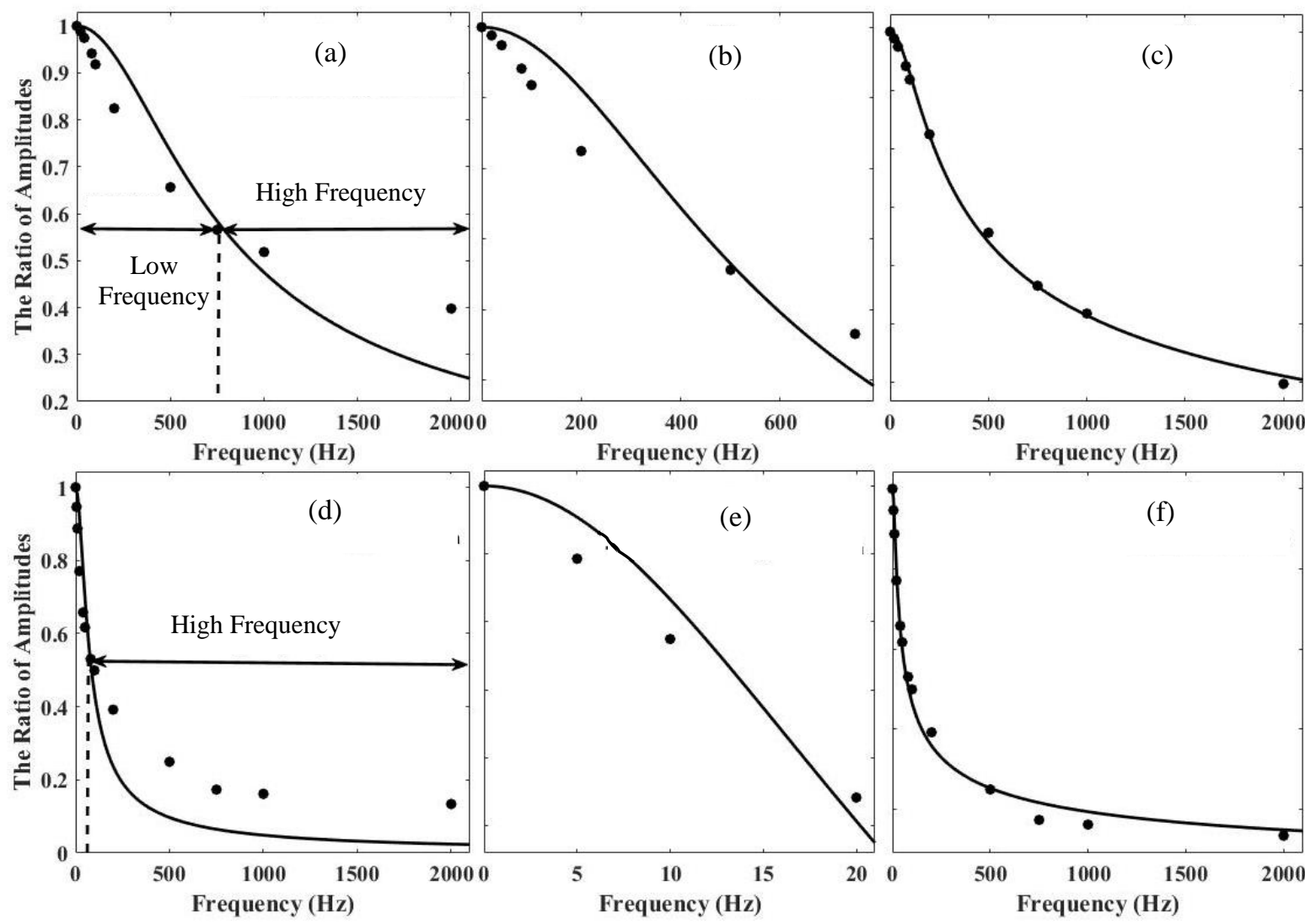

Fig. 4.4 Linear first order approximation for predicting transient response, (a) for the whole range of frequencies $(\varphi=1.0)$, (b) for the low range of frequencies $(\varphi=1.0)$, and (c) deviation from linear first order behavior $(\varphi=1.0)$. (d) The whole range of frequencies $(\varphi=0.7)$, (e) for the low range of frequencies $(\varphi=0.7)$, and (f) deviation from linear first order behavior $(\varphi=$ 0.7). Dots show numerical results and solid lines indicate nonlinear curve fit.

As shown by dashed lines in Fig. 4.4-a and 4.4-d, each plot is divided into two different zones, namely, low frequency and high frequency, which are different for each equivalence ratio. Linear first order approximation works well for the low frequency zone, but get progressively worse for the high frequency zone. The frequency at which this behavior changes is referred to as 
the cut-off frequency. As can be seen from Table 4.1, the cut-off frequency decreases with equivalence ratio. This behavior is mainly because of the lower burning rate in leaner flames. Table 4.1 compares the behaviour of each system in these two zones for three different equivalence ratios. Each equivalence ratio is divided into three domains, including a low frequency zone, high frequency zone, and all frequency data. $\tau$ represents the time constant of the first order model, $\boldsymbol{r}^{2}$ shows the accuracy of the first order model compared to time series data sets resulted from the numerical simulations, and $\xi$ illustrates the best-fit deviation from the linear model in each case. One important result from this table is that first order linear transfer functions can predict transient response with over $90 \%$ accuracy in the low frequency zone at each equivalence ratio. However, in the high frequency zone, the first order linear model fails to estimate the flame response. One possible reason could be the modifications of internal flame structure due to the approach of flow time scale to chemical time scale. 
Table 4.1 Comparison of linear model prediction accuracy and deviation from linear first order model for three different equivalence ratios of $0.7,0.8$ and 1.0.

\begin{tabular}{|c|c|c|c|}
\hline & \multicolumn{3}{|c|}{$\varphi=0.7$} \\
\hline & Lower than $20 \mathrm{~Hz}$ & $\begin{array}{c}\text { Higher than } 20 \\
\mathrm{~Hz}\end{array}$ & All Frequencies \\
\hline$\tau$ & 0.0061 & 0.0032 & 0.0023 \\
\hline$r^{2}$ & 0.9201 & 0.7344 & 0.9090 \\
\hline \multirow[t]{3}{*}{$\xi$} & 0.1354 & 0.2261 & 0.2005 \\
\hline & \multicolumn{3}{|c|}{$\varphi=0.8$} \\
\hline & Lower than $200 \mathrm{~Hz}$ & $\begin{array}{c}\text { Higher than } 200 \\
\mathrm{~Hz}\end{array}$ & All Frequencies \\
\hline$\tau$ & 0.0010 & 0.00052 & 0.00047 \\
\hline$r^{2}$ & 0.9901 & 0.7608 & 0.9566 \\
\hline \multirow[t]{3}{*}{$\xi$} & 0.1626 & 0.2574 & 0.2373 \\
\hline & \multicolumn{3}{|c|}{$\varphi=1.0$} \\
\hline & Lower than $750 \mathrm{~Hz}$ & $\begin{array}{c}\text { Higher than } 750 \\
\mathrm{~Hz}\end{array}$ & All Frequencies \\
\hline$\tau$ & 0.00058 & 0.00022 & 0.00025 \\
\hline$r^{2}$ & 0.9393 & 0.5389 & 0.9148 \\
\hline$\xi$ & 0.0967 & 0.1887 & 0.1517 \\
\hline
\end{tabular}

It should be noted that by using $\xi$, the quality of the fit improves significantly such that $r^{2}$ reaches $99 \%$.

\subsubsection{Linear Higher Order System Analysis}

In this section, in order to include flame behavior to a flow change in upstream of flame front, one needs to simulate each case for a wide range of frequencies and various input functions such as step, ramp, pulse, etc. This time-consuming process can be replaced by one or more transfer functions and the overall behavior of the flame can be defined using these functions. With regard to the application of this study, the input to the transfer functions are converted to stretch rate. The calculation of stretch rate is adopted from [83]. In this condition, the relationship between the stretch rate and heat release, which has been ignored in the current approach of flamelet modeling, 
reveals how flamelets respond to the time-dependent motion of a curved flame front. In order to have a better prediction of the transfer functions, a Polynomial Discrete-Time ARX Model [84], which is a generalized transfer function has been used. Equation (4.3) shows the general form of linear (due to the linear relationship between input and output) higher order (more than one term) transfer functions.

$$
C\left(z^{-1}\right) y(t)=\sum_{i=1}^{n u} D_{i}\left(z^{-1}\right) u_{i}\left(t-n k_{i}\right)
$$

In this equation, $u(t)$ is the input (stretch rate), $y(t)$ is the output (EI), $C$ and $D$ are polynomials expressed in the time-shift operator $z^{-1}, u_{i}$ is the $i^{t h}$ input, $n u$, the total number of inputs, and $n k_{i}$, the $i^{t h}$ input delay that characterizes the transport delay. In the present study, the system is SISO (single input/single output), therefore the transfer function takes a more familiar form wherein $i=1$. A Z-transform (Time-Discrete Fourier Transformation) can be used to convert the discrete-time form of the transfer function (Eq. 4.3) into a complex frequency domain representation.

This approach was first used to estimate the transfer function of the flame (with equivalence ratio of 0.8 ) response at $20 \mathrm{~Hz}$ fluctuations in mass flow rate. Then, the flame response to higher frequencies has been predicted using this transfer function. It has been observed that the accuracy of predictions decreases with increasing frequency. Increasing error in prediction of transient response in the low frequency zone confirms that nonlinearity of the system increases with increasing frequency at each equivalence ratio. However, it could be considered insignificant until the oscillation reaches the high frequency zone, where the intrinsic structure of the flame may be 
changed locally. In the next step, the whole range of low frequencies (frequencies lower than 200 $\mathrm{Hz}$, which is the cut-off frequency for equivalence ratio of 0.8 ) have been used to generate the transfer function (Eq. 4.4). Figure 4.5 shows that the accuracy of predictions is $\sim 88 \%$ and higher for frequencies up to $200 \mathrm{~Hz}$; however, the flame response experiences a significant drop in accuracy after $200 \mathrm{~Hz}$.

$$
\left.\begin{array}{l}
C(z)=1-0.2218 z^{-1}-0.4369 z^{-2}-0.3106 z^{-3}-0.03073 z^{-4} \\
D(z)=-6.712 e 06 z^{-1}+2.026 e 07 z^{-2}-2.039 e 07 z^{-3}+6.843 e 06 z^{-4}
\end{array}\right\}
$$
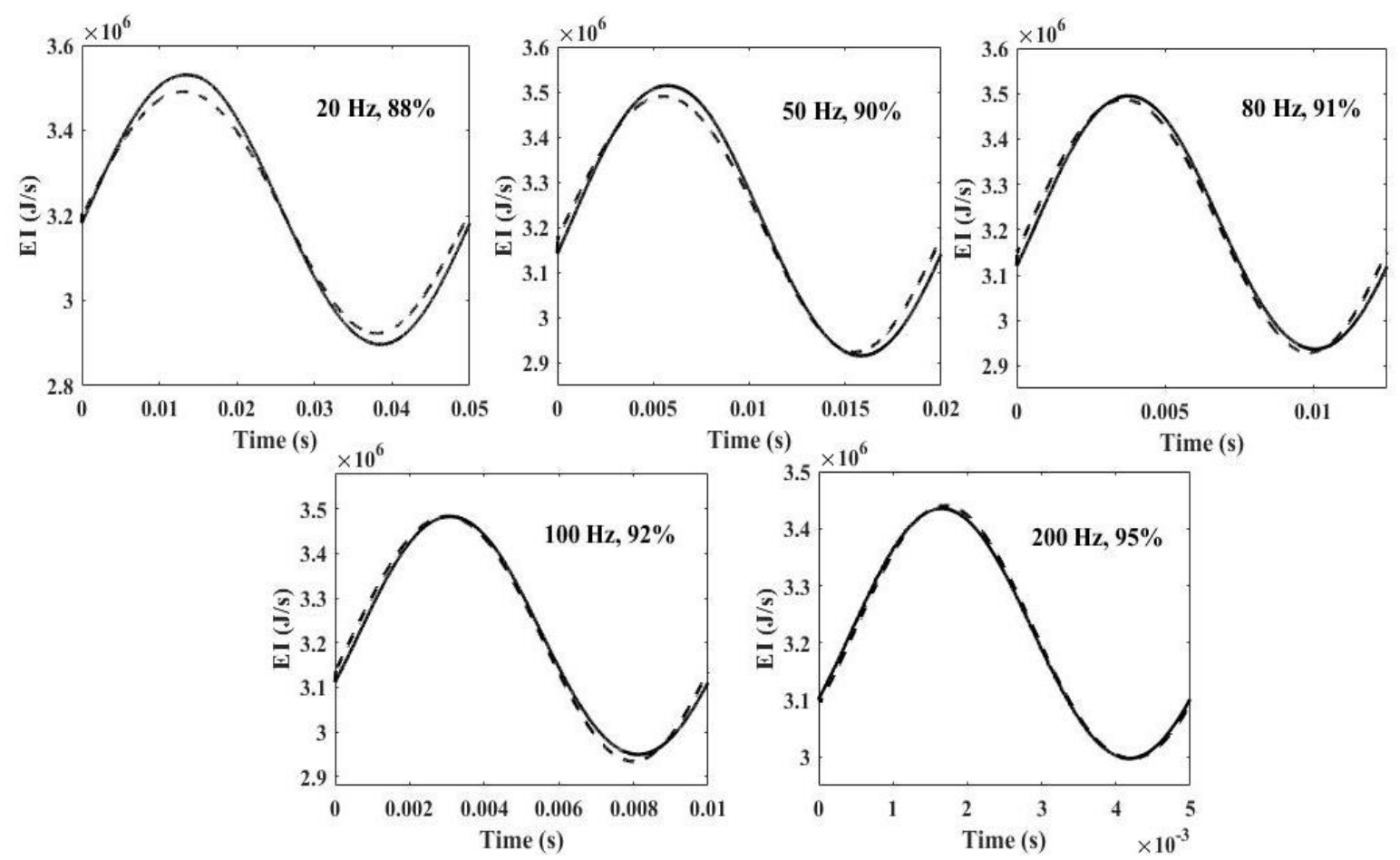

Fig. 4.5 Estimation of transient response of a laminar premixed flame for $\varphi=0.8$ based on low frequency data using a higher order linear model (ARX model)

$C(z)$ and $D(z)$ are the expressions represented in Eq. 4.3. The fourth order transfer function means that a minimum of the first four terms on each side of Eq. 4.3 is required to have the most 
accurate predictions. It should be noted that $C(z)$ and $D(z)$ are usually referred to as the poles and zeros of a transfer function in the literature.

Based on the higher order linear model, a transfer function (Eq. 4.5) is generated for predicting the high frequency zone transient response. Figure 4.6 illustrates the accuracy of this model for frequencies higher than $200 \mathrm{~Hz}$.

$$
\left.\begin{array}{l}
C(z)=1-0.8901 z^{-1}-0.5892 z^{-2}+0.1596 z^{-3}+0.3196 z^{-4} \\
D(z)=-1161 z^{-1}+3533 z^{-2}-3594 z^{-3}+1226 \mathrm{e} 06 z^{-4}
\end{array}\right\}
$$
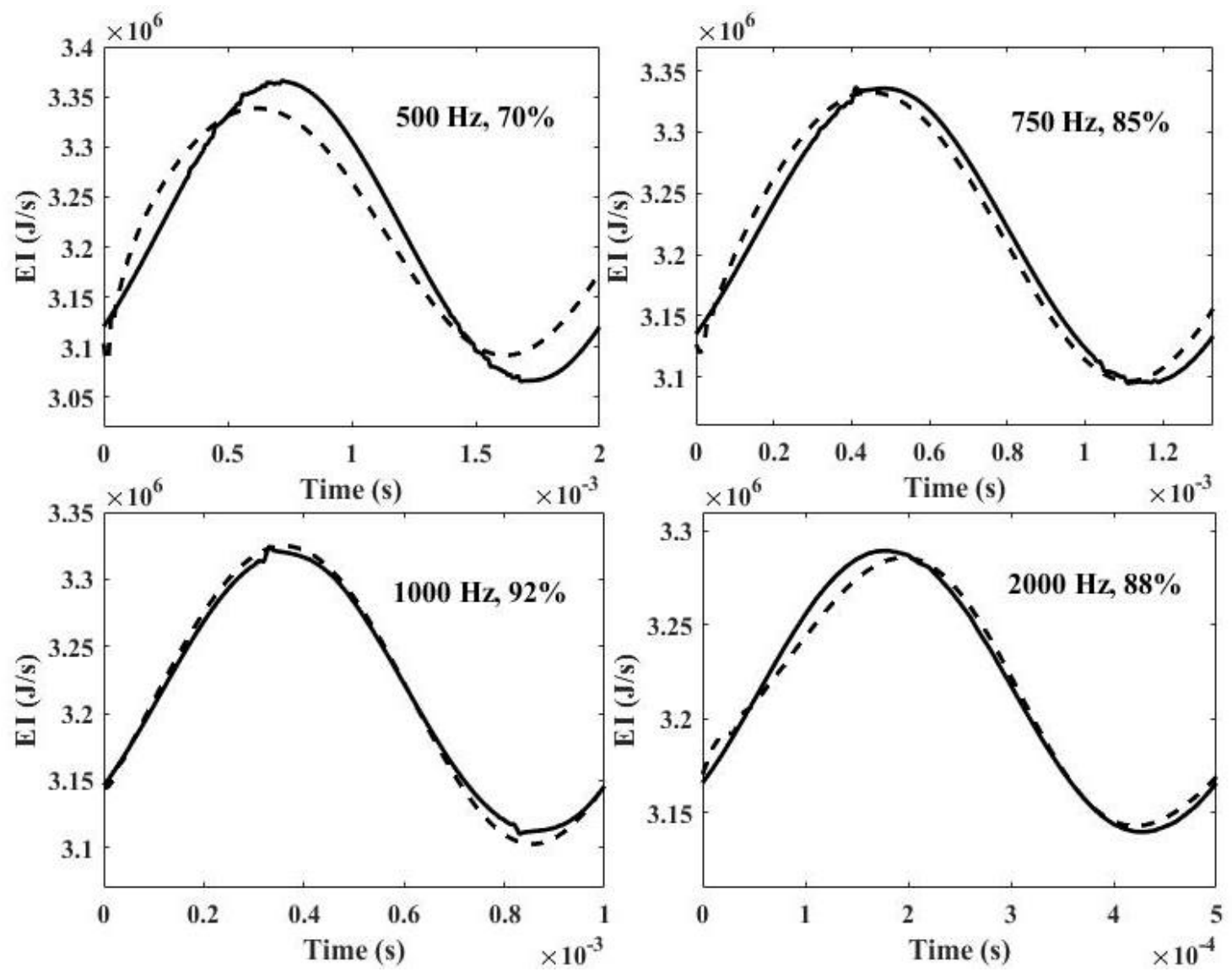

Fig. 4.6 Estimation of transient response of a laminar premixed flame for $\varphi=0.8$ based on high frequency data using higher order linear model (ARX model) 
In order to study the effect of the amplitude on flame behavior and the transfer function, the ratio of the oscillations to the mean value was doubled. The results in Eq. (4.6) depict a similar fourth order transfer function, which predicts the high frequencies $(70 \%$ for $500 \mathrm{~Hz}, 84 \%$ for 1000 $\mathrm{Hz}$, and $91 \%$ for $2000 \mathrm{~Hz}$ ), with different coefficients.

$$
\left.\begin{array}{l}
C(z)=1-0.8968 z^{-1}-0.5579 z^{-2}+0.04589 z^{-3}+0.4088 z^{-4} \\
D(z)=-228.6 z^{-1}+727.4 z^{-2}-766.1 z^{-3}+270.5 \mathrm{e} 06 z^{-4}
\end{array}\right\}
$$

The fourth order equation also indicated the effective transfer function for various equivalence ratios. For example, a stoichiometric flame was tested with a similar geometry, flame curvature, and amplitude. As can be seen in Eq. (4.7), the optimum transfer function has the same format as the other cases except the coefficients. In other words, in order to predict the output of a flame with a different amplitude, a fourth order transfer function can be used; however, the coefficients need to be altered. The accuracy for the high range of frequencies is $83 \%$ for $500 \mathrm{~Hz}$, $93 \%$ for $1000 \mathrm{~Hz}$, and $94 \%$ for $2000 \mathrm{~Hz}$. The higher accuracy in the same frequencies was expected due to the faster burning rate at the stoichiometric condition compared to the lean flames.

$$
\left.\begin{array}{l}
C(z)=1-0.4891 z^{-1}-0.3808 z^{-2}-0.08371 z^{-3}-0.04643 z^{-4} \\
D(z)=-6461 z^{-1}+2.017 e^{4} z^{-2}-2.111 e^{4} z^{-3}+270.5 \mathrm{e} 06 z^{-4}
\end{array}\right\}
$$

As can be concluded from the recent results in this section, the whole range of frequencies is divided in two sections based on the cut-off frequency obtained from numerical simulations of transient response of laminar premixed flames for each equivalence ratio and a separate transfer 
function is defined for each section. Although the accuracies are in an acceptable range, there may be a considerable improvement using nonlinear models to predict the output. Therefore, in order to study the capability of nonlinear models to predict the flame response more accurately, different nonlinear transfer functions were analyzed in the next section.

\subsubsection{Nonlinear System Analysis}

As shown in the previous section, although linear transfer functions can achieve accurate prediction in the low frequency range of flame response to changes in stretch rate, they are not able to predict the response at some high frequencies. As mentioned in the introduction, in analyzing flame instabilities, flow velocity perturbations (as the input to the system) change the heat release rate (as the output) by influencing the flame surface area. Therefore, flame transfer functions are studied using the flame response to velocity fluctuations. The heat release rate modulations result in acoustic pressure perturbations, and could be linked to inlet flow velocity oscillations. Therefore, in order to capture the nonlinearities in the thermos-acoustic coupling, the flame transfer function has been replaced by the flame describing function [85]. It has been reported that in premixed combustion systems, the relationship between velocity and heat release makes the system nonlinear whereas gas dynamic processes generally stay in the linear regime [86].

One difference between linear and nonlinear transfer functions is in the representation of the equivalent time-domain. Nonlinear systems are defined based on a sequence of transfer functions, as opposed to only one function in the linear case. Although with stronger nonlinearities, more transfer functions are required to study the behavior of the system accurately, for a wide range of nonlinear systems, it is usually sufficient to capture the dominant effects by considering the first, second, and third order transfer functions. The other difference is that even in a single 
input/single output nonlinear system, a transfer function is multi-variate, which increases the difficulty of defining a transfer function to relate the system response to input, interpret the output, and obtain complete understanding of its behavior. In this regard, it could be useful to apply Nonlinear Frequency Response Analysis (NFRA) on the current transient data.

Nonlinear frequency response analysis, like a first order linear system, applies a sinusoidal function as the input. However, the system cannot be considered linear around the steady state condition. In this case, nonlinear perturbations occur, and therefore, similar to the flame analyzed in this chapter, the transient response can no longer be expressed with a single sinusoidal function. New frequencies such as harmonics and intermodulation frequencies are generated, meaning that the sinusoidal functions of the high frequency response data can be best approximated by a sum of two or three sinusoidal functions instead of one. The fact that the current time-series data in the high frequency zone could be approximated by the sum of two or three sinusoidal functions more accurately indicates this aspect of nonlinear systems for laminar premixed flames. These perturbations can be studied by higher order frequency response functions, which is a mathematical framework, such as a Volterra series [87]. The advantage of using a higher order transfer function is that it contains information about the nonlinearities of the system. Equation (4.8) shows the general form of nonlinear systems in the time domain.

$$
y(t)=\sum_{n=1}^{\infty} y_{n}(t)=\sum_{n=1}^{\infty} \int_{-\infty}^{\infty} \ldots \int_{-\infty}^{\infty} h_{n}\left(\tau_{1}, \ldots, \tau_{n}\right) \prod_{i=1}^{n} u\left(t-\tau_{i}\right) d \tau_{i}
$$

$y(t)$ is the output in the time domain. Applying Fourier series, the output can be estimated using the nonlinear transfer function in the frequency domain (Eq. 4.9). 


$$
Y_{n}\left(s_{1}, \ldots, s_{n}\right)=H_{n}\left(s_{1}, \ldots, s_{n}\right) U\left(s_{1}\right) \ldots U\left(s_{n}\right)
$$

With this form of the transfer function, a value of one for $n$ results in a first order system. Notice that both the impulse response function and the transfer function are independent of the input excitation. This is a highly desirable feature because it enables the determination of the system response for arbitrary inputs, as will be shown in the last section of this chapter.

Although higher order linear systems and nonlinear systems have multidimensional transfer functions, their behavior is significantly different. Higher order linear systems have real multidimensional input/output that are defined with integer arguments. There are different approaches to model nonlinear systems including Volterra series models, block structured models (Wiener and Hammerstein [88]), neural network models, and nonlinear auto-regressive exogenous (NARX) models, and the nonlinear auto-regressive moving average model with exogenous inputs (NARMAX) [89].

Among these predictive models, NARX showed better results in estimating the transient response of the flame for the whole range of frequencies including low and high frequency zones. NARX models (which is an extension of ARX in polynomial transfer functions) are flexible nonlinear functions that are able to capture the complex behavior in nonlinear systems such as the current data set. Eq. (4.10) illustrates the structure of a linear Single Input/Single Output ARX model:

$$
\begin{aligned}
y(t) & +a_{1} y(t-1)+a_{2} y(t-2)+\cdots+a_{n a} y(t-n a) \\
& =b_{1} u(t)+b_{2} u(t-1)+\cdots+b_{n b} u(t-n b+1)+e(t)
\end{aligned}
$$


where $u, y$ and $e$ are the input (stretch rate), output (EI), and noise (zero in this study), respectively. This structure implies that the output $y(t)$ in current time is predicted as a weighted sum of past output values and current and past input values. $n a$ is the number of past output terms, and $n b$ is the number of past input terms used to predict the current output. It has been shown that the surface wrinkling in turbulent flames at one location, in addition to the local velocity perturbation, depends also on the flame surface fluctuations at previous times upstream of the flame front [90]. Therefore, an ARX model could capture this memory effect occurring in turbulent flames by modifying the laminar flamelet response to a change in upstream flowrate. Rewriting Eq. (4.11) as a product gives:

$$
\begin{gathered}
y_{p}(t)=\left(-a_{1},-a_{2}, \ldots,-a_{n a}, b_{1}, b_{2}, \ldots, b_{n b}\right)(y(t-1), \\
y(t-2), \ldots, y(t-n a), u(t), u(t-1), \ldots, u(t-n b-1))
\end{gathered}
$$

where $y(t-1), y(t-2), \ldots, y(t-n a), u(t), u(t-1), \ldots, u(t-n b-1)$ are delayed input and output variables, called regressors. The coefficients vector $\left(-a_{1}, \ldots,-a_{n a}, b_{1}, \ldots, b_{n b}\right)$ represents the weighting applied to these regressors. The linear ARX model thus predicts the current output $y_{p}$ as a weighted sum of its regressors. The flexibility of the nonlinear ARX model allows for replacing the weighted sum of the regressors in a linear model with one of several available nonlinear functions, $\mathcal{M}$.

$$
y_{p}(t)=\mathcal{M}(y(t-1), y(t-2), y(t-3), \ldots, u(t), u(t-1), u(t-2), \ldots)
$$


The nonlinearity estimator $(\mathcal{M})$ for calculating the nonlinear ARX model in this study was wavenet, which stores the wavelet network [91] for use in transfer functions. Different parameters of this network are automatically estimated in the system identification toolbox of MATLAB.

In addition to simple delayed input-output variables, a more complex nonlinear expression of delayed input and output variables can be used in nonlinear ARX regressors. This type of nonlinear transfer function consists of regressors and a nonlinearity estimator. In the nonlinearity estimator, linear and nonlinear functions can be applied to the model regressors to predict the system output.

After applying the nonlinear ARX model to predict the flame response, the results need to be validated by comparing the estimations with time series data sets obtained from complex chemistry numerical simulations. The nonlinear model shows high accuracy for low frequency zone (Fig. 4.7), but also predicts the results for higher frequencies reasonably well (Fig. 4.8). The frequency has been increased up to $2000 \mathrm{~Hz}$ in this study. 

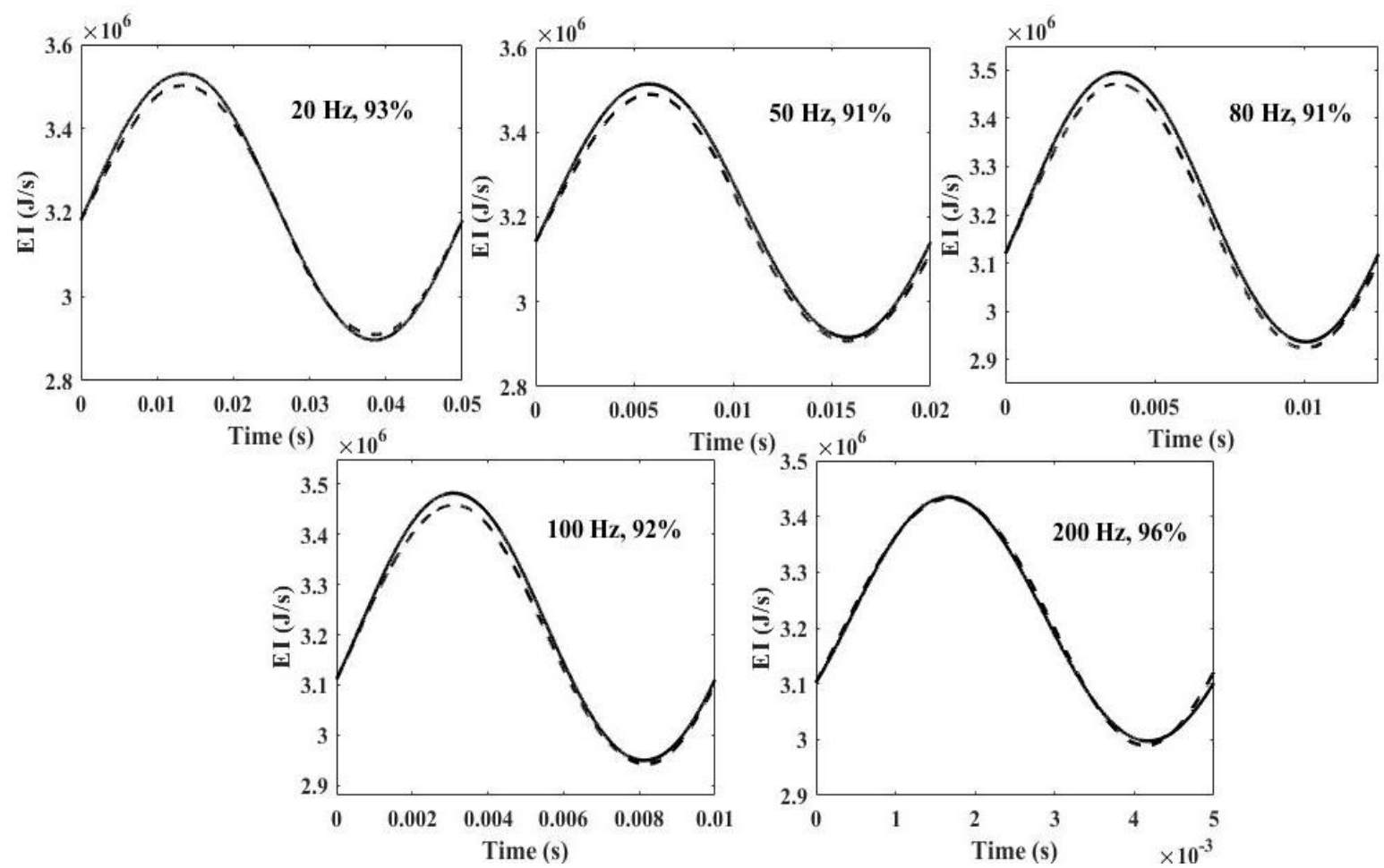

Fig. 4.7 Estimating of transient response of a laminar premixed flame for $\varphi=0.8$ in low frequency zone using a nonlinear model (NARX)
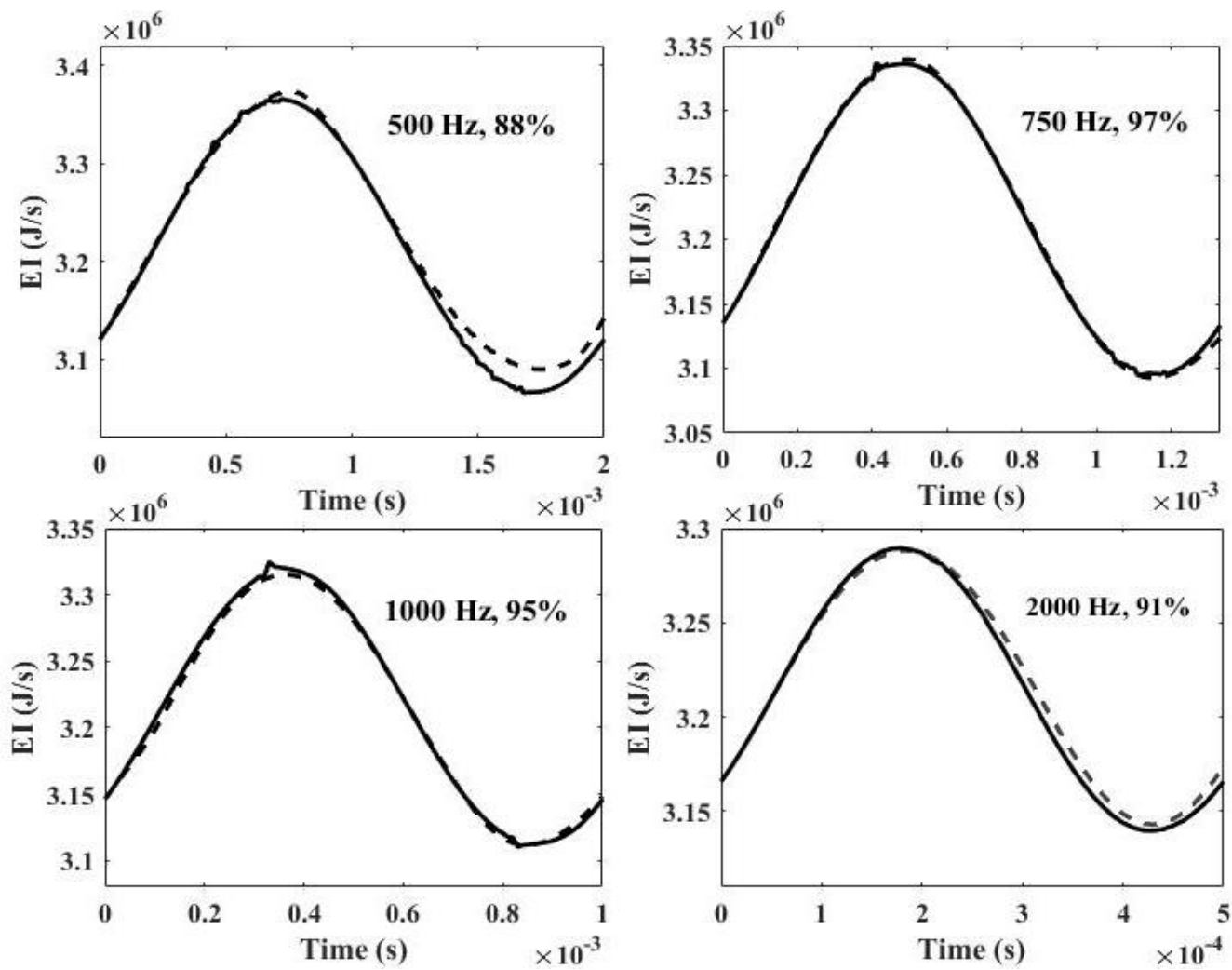

Fig. 4.8 Estimating of transient response of a laminar premixed flame for $\varphi=0.8$ in high frequency zone using a nonlinear model (NARX) 
In Fig. 4.8, a few sharp discontinuities can be observed on the top and bottom of the plots. Although, it has been shown previously that the time stepping and the grid spacing are essentially sufficient to capture all the physical and chemical changes in the flame, a further numerical consideration is needed to adapt the correct time step and grid size in those regions. These discontinuities are observed in the regions when the flame reaches maximum or minimum positions in a cycle and are mainly due to the delay in flame response that change the flame structure.

\subsection{Prediction of a Random Input Response Using Transfer Functions}

Turbulent flows consist of flow perturbations that are not simple sinusoidal functions. For our purposes here these fluctuations were modelled as a sum of different sinusoidal functions with different amplitudes, frequencies, and phase lags. The transfer functions developed in the previous sections can be used to predict the transient responses to a random-looking input. In order to test this capability, three different ranges of frequency were selected including low, intermediate and high frequency range. First, four frequencies are used to form the input in the low frequency zone by summing them. Frequencies within the low range are selected $(20 \mathrm{~Hz}, 50 \mathrm{~Hz}, 80 \mathrm{~Hz}$, and 100 $\mathrm{Hz}$ ) which results in forming a stretch rate perturbation in Fig. 4.9 (top figure). This input was used

to compare different responses including the quasi-steady response, and the response resulting from detailed numerical calculations (Fig. 4.9 - bottom figure). The solid line curve indicates the quasi-steady response in which there is no amplitude change or phase lag in the output of the system. The dashed line curve shows the numerical results obtained from the code based on full complex chemistry, which was originally used to calculate the transient response at each individual case. 

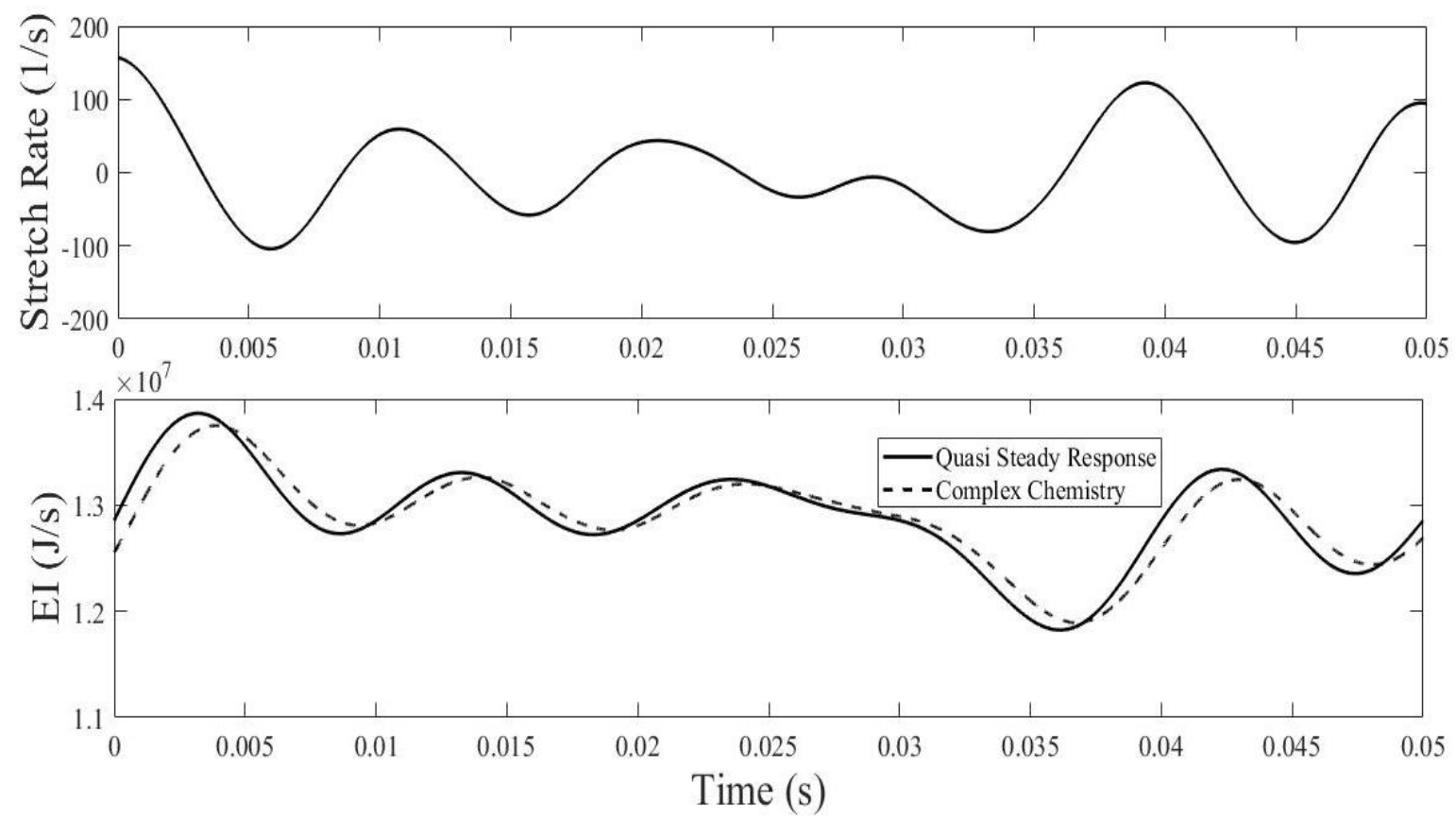

Fig. 4.9 Comparison of quasi-steady and transient response (complex chemistry) of a laminar premixed flame to a random input in low frequency range $(\varphi=0.8)$, Top figure: stretch rate perturbation as the input, Bottom figure: solid line the quasi-steady response and dashed line the transient response (complex chemistry).

A comparison has been made in order to show the similarity of the transfer function output to the complex chemistry transient response (Fig. 4.10). In Fig. 4.10, the transient response is illustrated by the solid line curve and the transfer function output is shown by dashed line. The accuracy of the model (defined by Eq. 4.13) is $90 \%$, which shows the output variations that is reproduced by the model while the quasi-steady response shows $75 \%$ accuracy compared to the time-dependent numerical simulation.

The accuracy of the fit is defined as follows:

$$
F i t=\left(1-\frac{\operatorname{norm}(E I-\overline{E I})}{\operatorname{norm}(E I-\operatorname{mean}(E I))}\right) \times 100
$$




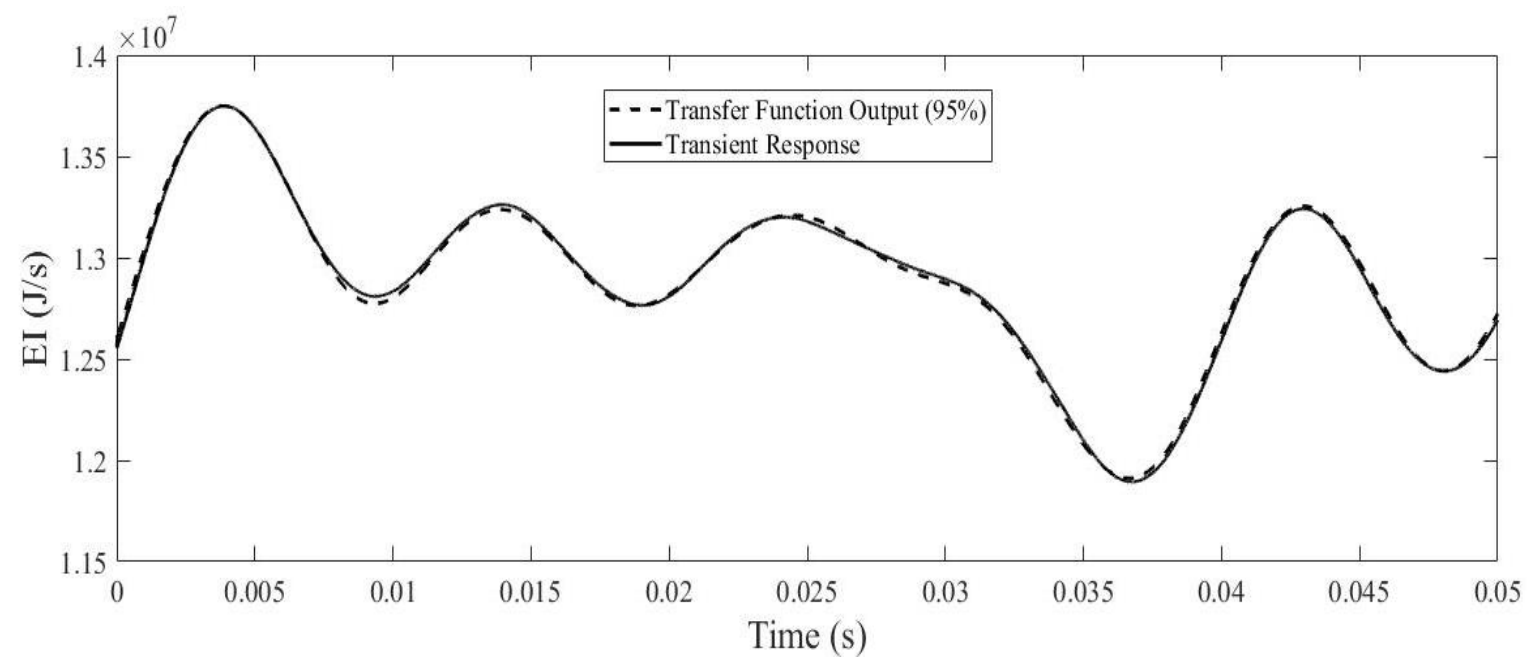

Fig. 4.10 Comparison of transient response of a laminar premixed flame and nonlinear model output in low frequency range for $\varphi=0.8$

The same approach was taken to predict the response using the transfer function in the previous section for high frequency zone $(500 \mathrm{~Hz}, 1000 \mathrm{~Hz}$, and $2000 \mathrm{~Hz})$. As depicted in Fig. 4.11, in this condition, the deviation of quasi-steady response from the complex chemistry transient simulation is more significant compared to low frequency analysis. The accuracy of fit in this case is only $25 \%$. As shown in Fig. 4.12, the transfer function that was obtained from the high frequency data, is capable of increasing this accuracy to $84 \%$. 

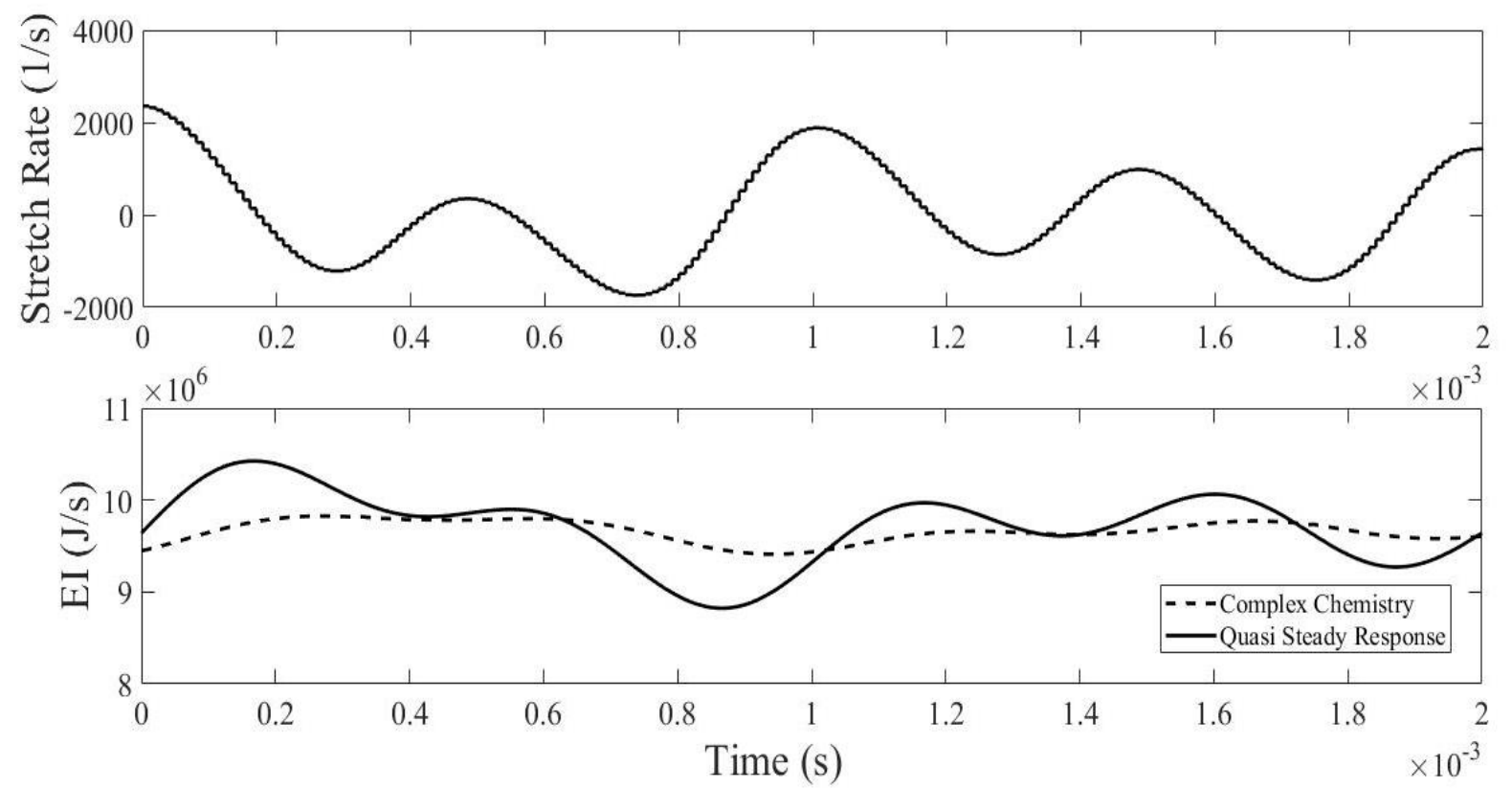

Fig. 4.11 Comparison of quasi-steady and transient response (complex chemistry) of a laminar premixed flame to a random input in high frequency range $(\varphi=0.8)$, Top figure: stretch rate perturbation as the input, Bottom figure: solid line the quasi-steady response and dashed line the transient response (complex chemistry).

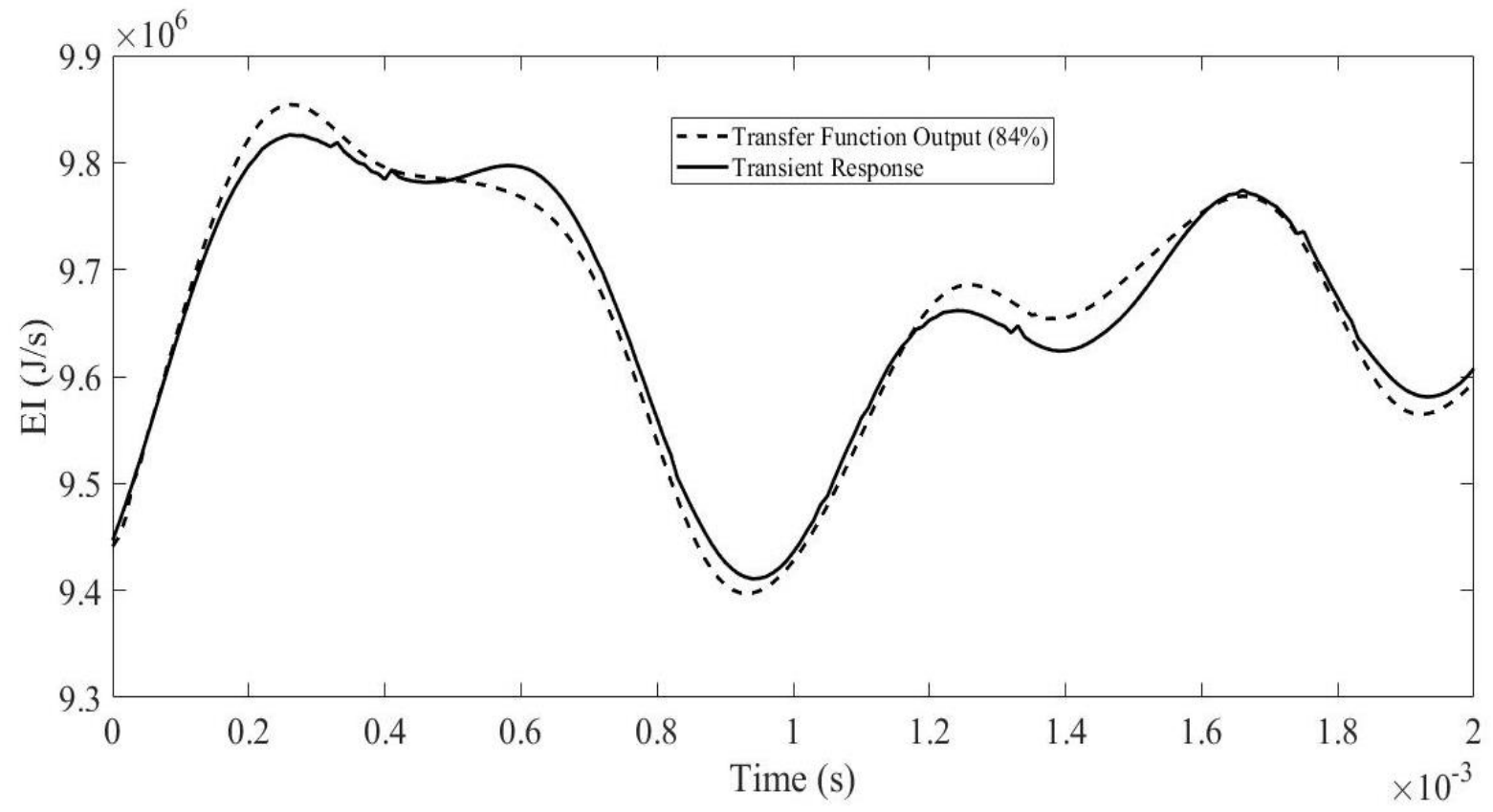

Fig. 4.12 Comparison of transient response a laminar premixed flame and nonlinear model output in high frequency range for $\varphi=0.8$ 
In the intermediate range, a model was developed based on both low and high frequency datasets. In order to test this model, a combination of low and high frequencies $(80 \mathrm{~Hz}, 100 \mathrm{~Hz}$, $200 \mathrm{~Hz}$, and $500 \mathrm{~Hz}$ ) were selected. As can be observed from Fig. 4.13 the quasi steady response is $30 \%$ similar to complex chemistry resulted from transient numerical simulation while transfer function is capable of producing the transient results with $74 \%$ accuracy (Fig. 4.14).

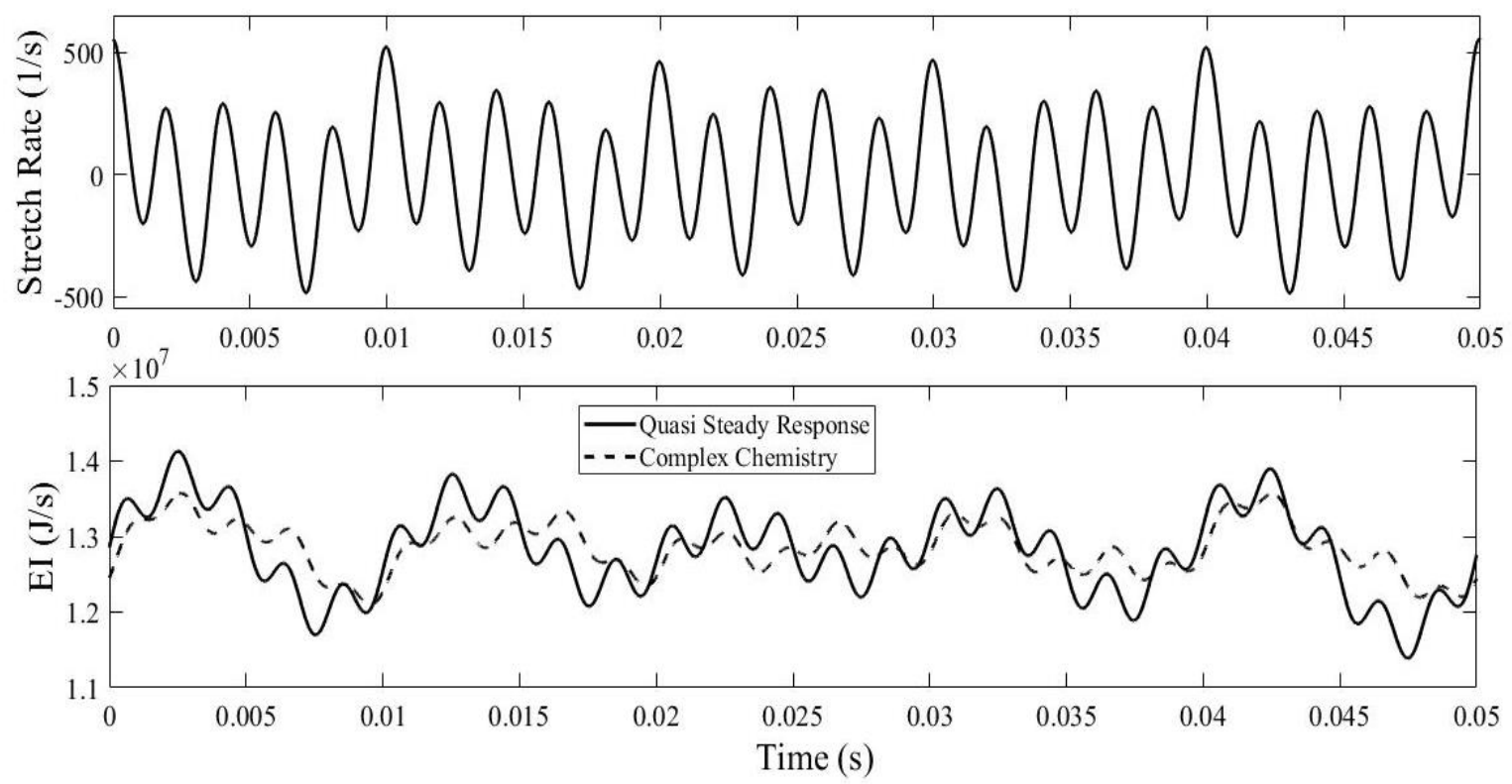

Fig. 4.13 Comparison of quasi-steady and transient response (complex chemistry) of a laminar premixed flame to a random input in intermediate range $(\varphi=0.8)$, Top figure: stretch rate perturbation as the input, Bottom figure: solid line the quasi-steady response and dashed line the transient response (complex chemistry). 


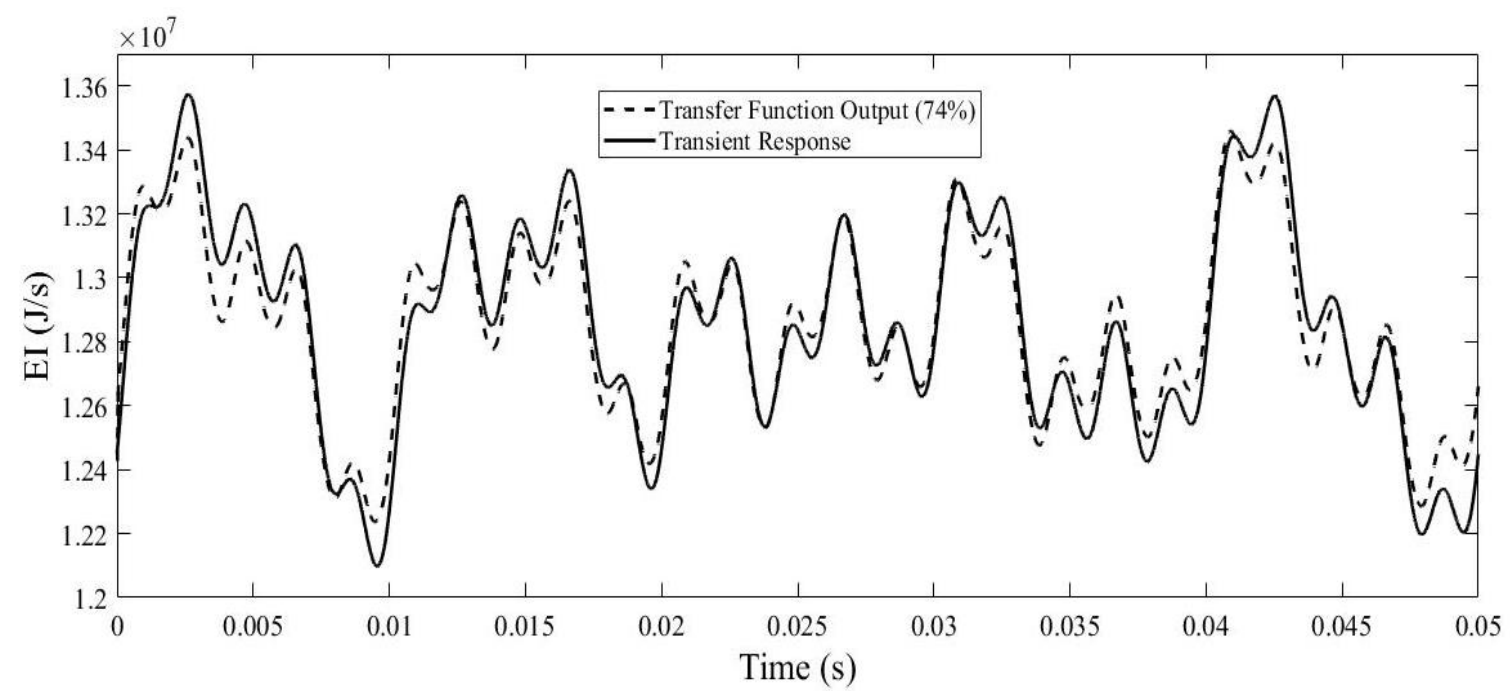

Fig. 4.14 Comparison of transient response of a laminar premixed flame and nonlinear model output in intermediate range for $\varphi=0.8$

Comparing the three cases explained above, one understands that low and high frequency zones can be predicted accurately using a transfer function generated for each separate range. Although the intermediate frequency range does not show the high accuracy compared to the other cases, it shows an acceptable improvement compared to the quasi-steady response.

In summary, laminar premixed flames are complicated systems containing multiple heat and mass transfer and chemical processes. Nonlinearity results from the coupling of these processes. Therefore, in this nonlinear system, the transfer function, which shows the relationship between the mass flowrate fluctuations and the energy release rate oscillations at different frequencies, will not accurately behave as a linear first order transfer function. However, the linear first order transfer function provides a helpful tool with a simple visual representation and a clear understanding of system behavior for laminar premixed flame response in the low frequency zone. Although, the ability to obtain transfer functions for nonlinear practical systems such as laminar flame response is limited due to the difficulties in presentation and interpretation of these systems, applying the NARX model showed an improvement in estimating the flame response to a change 
in upstream flow velocity or stretch rate. Therefore, it would be very desirable to have such a mathematical framework to estimate the flame response to any change in upstream flow velocity or stretch rate instead of time-consuming numerical simulations.

In order to implement the results in a flamelet model, the idea presented in this research is to apply the transfer functions to modify the flame energy release according to the flame instantaneous response in a transient condition. The LES framework uses flamelet models based on reaction progress variables which can be used as the input to transfer functions [81]. However, the definition of the progress variables is not consistent between different models. Therefore, in this study, in order to include the transient effects in a more general way, the stretch rate has been selected as the input to the system. Furthermore, using stretch rate as the input to the mathematical model can be more useful to extract insights from the transient results. Thus, with regard to the definition of the scalar quantity that is being used to describe the flame in flamelet libraries, the input to the transfer functions can be altered.

\subsection{Conclusions}

In this chapter, the transient response data on the rate of conversion of chemical to sensible enthalpy for a laminar premixed flame that was exposed to a periodic flow conditions were analyzed in order to estimate a transfer function between these inputs and outputs. The study of the transient response analysis of a curved flame could be a potential modification to quasi-steady assumption in a laminar flamelet library in order to improve premixed turbulent modeling. The transient response dataset was taken from a previous study, which was a complex chemistry simulation of an inwardly burning laminar premixed (lean methane / air) flame in a cylindricallysymmetric geometry for different equivalence ratios $(0.7-1.0)$ and cyclic exposure to positive and 
negative stretch rates at different frequencies $(0-2000 \mathrm{~Hz})$. In this chapter, the higher order linear and nonlinear analysis have been only performed for equivalence ratio of 0.8 to show the capability of transfer functions to capture the flame behavior when exposed to a flow perturbation in upstream of the flame front.

A linear first order model was applied to the data and it fit the low frequency results for each flame. However, the flame behavior deviates from that of a linear first order system as the frequency is increased approached conditions where the chemical and flow time scales were the same. Applying higher order linear transfer functions did not significantly improve the comparison due to stronger nonlinearities at high frequencies.

Nonlinear models, such as the nonlinear ARX model, could correctly predict the transient heat release over the whole range of frequencies of changing flow conditions. This transfer function could also reconstruct the heat release response for any arbitrary flow input, which enables the model to capture the transient effects of a flame in any conditions. Therefore, estimating a flame transfer function of laminar premixed flames could yield a better understanding of flame dynamics and could be used in order to modify the quasi-steady assumption in laminar flamelet models of turbulent combustion. This has been confirmed by applying various random-look functions for stretch rate as the input to system for low, intermediate, and high frequency zones. Using the generated transfer functions based on the simulation datasets, the transient complex chemistry results were predicted with a relatively good accuracy. Thus, the effect of the unsteady stretch rate on the heat release due to chemical conversion of the reactants to products can be stored in a series of transfer functions. These functions can be recalled in a flamelet library to be applied to the steady pre-calculated flames with the same properties such as temperature, fuel type, equivalence ratio, and pressure. 


\section{Chapter 5: Summary and Conclusions}

\subsection{Summary}

This dissertation was mainly focused on the transient response of a laminar premixed flame to sinusoidal perturbations in mass flow rate in order to study the effects of the motion of a curved flame front in producing stretch, separate from the strain rate component. In Chapter 2, a onedimensional numerical algorithm was developed in order to simulate an axi-symmetric timedependent laminar premixed flame that propagates in the radially inward direction. The transient governing equations of continuity, momentum, energy, and species mass fractions were solved simultaneously using a modified Newton's method with a Bi-CGSTAB linear solver. A finite volume technique was used to discretize these governing equations. Initially, for a specific flame (i.e., specified fuel type, equivalence ratio, mean flame curvature, and mass flow rate), a steady state solution was obtained using steady boundary conditions. In order to validate the steady solution, the composition of the products were compared to the equilibrium state obtained from equilibrium thermodynamics for an adiabatic process, and the flame burning rate was compared to the experimental results for an unstretched planar flame. The computational results for three different flames (stoichiometric and two lean flames) had good agreement with the aforementioned thermodynamics and experimental data. In the next step, by changing the boundary conditions to transient mode, the steady flame was introduced as the initial condition in order to study the flame behavior in an oscillating flow.

In chapter 3, the model was used to expand the study to revisit the quasi-steady assumptions that are used in current approaches of premixed laminar flamelet models. In this regard, a sinusoidal variation of mass flow rate at the inlet boundary with specifiable frequency was applied to investigate the transient response of a laminar premixed flame. The amplitude of variation of 
mass flow rate was $10 \%$ of the mean flow rate (though some tests were done at $20 \%$ ). This transient model when the fluctuating amplitude was set to $0 \%$ was validated against stationary flame results. Since the stationary flame in this geometry is not stretched but has a finite curvature, an investigation was made to compare key characteristics of steady unstretched flames to the dynamics of a stretched flame due to the motion of the curved flame front. The delayed response illustrated the fact that the flame cannot respond to a change in upstream mass flow rate or flow fluctuations instantaneously, even when the flow and scalar fields align (i.e., no tangential strain). It was observed that while the flame either expands (positive stretch) or contracts (negative stretch), the flame indicated distinct behavior. The parameter used for this comparison was the total conversion of chemical enthalpy to thermal enthalpy across the flame, $E I^{\prime}$. The flame response was investigated for equivalence ratios of 1.0, 0.8, and 0.7 and the frequencies up to 2000 Hz. The amplitude of oscillations in mass flow and mean flame curvature were kept constant.

In Chapter 4, the transient response data on the rate of conversion of chemical to sensible enthalpy for a laminar premixed flame that was exposed to periodic flow conditions was analyzed in order to estimate a transfer function between these inputs and outputs. The transient response dataset that was taken from the previous chapter was completed by adding more data on a different amplitude and mean flame curvature. In this chapter, first, a first order linear study was performed for equivalence ratios of 1.0, 0.8, and 0.7 which showed relatively good results for low frequencies. Then, the higher order linear and nonlinear analysis was performed for the equivalence ratio of 0.8 to show the capability of higher order transfer functions to capture the flame behavior when exposed to a flow perturbation upstream of the flame front. The flame response was divided into low and high frequency zones the boundary of which was referred to as the cut-off frequency. It was observed that the cut-off frequency is unique for each flame with different equivalence ratio. 
In the end, transfer functions were tested by three cases with different random flow fluctuations.

The results indicated significant improvements compared to quasi steady responses.

\subsection{Conclusions}

The main conclusions of this dissertation are:

1. It has been concluded that similar to planar flames exposed to oscillating strain rates, where the flame stretch always has a positive value, the transient response of laminar premixed flames results in decreasing amplitudes of motion and burning rates and increasing phase lag with increasing frequency. This changing $E I^{\prime}$ amplitude and phase lag resulted in an elliptical shape when it was plotted with respect to the instantaneous mass flow rate, which showed differing burning rates between positively and negatively stretched flames with the same imposed instantaneous hydrodynamic state, as well as how it differed from the quasi-steady state.

2. The model that was proposed and developed in this thesis indicated how expanding (positive stretch), contracting (negative stretch), and steady flames (unstretched) can behave differently at various frequencies and equivalence ratios in terms of chemical energy release. This phenomenon stresses the fact that in order to generate a more rigorous flamelet library to model a premixed turbulent flame, the timedependent motion of a curved flame should be considered in both positive and negative stretch rates.

3. A linear first order model was applied to the data and it fit the low frequency results for each flame. However, the flame behavior deviated from that of a linear 
first order system as the frequency was increased and approached conditions where the chemical and flow time scales were the same. Applying higher order linear transfer functions improved the predictions. Changing variables such as frequency, mean flame stretch, equivalence ratio, and amplitude did not affect the general format of the transfer functions and only the coefficients were altered in different cases.

4. Nonlinear models could correctly predict the transient heat release over the whole range of frequencies of changing flow conditions more accurately. These transfer functions could also reconstruct the heat release response for any arbitrary flow input, which enables the model to capture the transient effects of a flame in any conditions. Therefore, estimating a transfer function of laminar premixed flames could yield a better understanding of flame dynamics and could be used in order to modify the quasi-steady assumption in laminar flamelet models of turbulent combustion. This observation has been confirmed by applying various random functions for stretch rate as the input to the system for both low and high frequency zones. Using the transfer functions generated based on the simulation datasets, the transient complex chemistry results indicated a significant improvement compared to quasi-steady response.

\subsection{Contributions}

The novel contributions of this dissertation can be summarized as:

1. A FORTRAN code was developed, based on first principles, and implemented for simulating time-dependent laminar premixed flames in a cylindrically symmetric 
geometry with steady and transient boundary conditions. The configuration generates both positive and negative stretch rate while the strain rate effect does not exist. [M. Sahafzadeh, L. W. Kostiuk, S. B. Dworkin, “A Numerical Algorithm for Modeling Laminar Premixed Flames Exposed to an Oscillating Flow Field in CylindricallySymmetric Geometry," Submitted to Computer Physics Communications, (under review)]

2. Using a sinusoidal variation of mass flow rate at the inlet boundary of the steady flame, a novel approach was introduced which facilitated distinguishing between the two components of stretch rate, namely strain rate and motion of a curved flame. This approach provided a consistent means to determine the effect of various parameters such as equivalence ratio, frequency, amplitude, and mean flame curvature on the flame response. [M. Sahafzadeh, L. W. Kostiuk, S. B. Dworkin, "Transient response of a laminar premixed flame to a radially diverging/converging flow," Combustion and Flame, vol. 179, pp. 51-62, May 2017]

3. The laminar premixed flame response was divided into two main regions, namely low and high frequency zones. The frequency at which the flame behavior changes is a function of fuel type and equivalence ratio. This behavior occurs when flow time scale approaches the chemistry time scale. Therefore, a definition of Damköhler number which is the ratio of these time scales can be used as a universal dimensionless parameter for this system.

4. For the first time, a frequency response analysis was used to capture the transient response of a laminar premixed curved flame in a transfer function. With regard to the applicability of this research to flamelet modeling, the input to the transfer function 
was considered to be stretch rate fluctuations and the output was kept the same as energy release.

5. It was shown that a fourth order ARX model could be used to predict the transient effects of flow perturbations upstream of the flame front in most cases. However, nonlinear models are capable of predicting the output in a wider range of frequencies. Among all the examined nonlinear models, the nonlinear ARX model with a wavelet network block illustrated more promising results.

6. In general, the higher order and nonlinear transfer functions, due to their mathematical structure which saves the time history of the inputs and outputs, are capable of capturing the time dependent effects in laminar premixed flames. [M. Sahafzadeh, S. B. Dworkin, L. W. Kostiuk, "Using frequency response analysis to predict unsteady burning rate from unsteady stretch rate," submitted to Combustion and Flame, (under review)]

\subsection{Recommendations for Future work}

The research presented in this dissertation has the potential to be extended in the following aspects:

1. The numerical simulation code can be improved by applying a second order temporal discretization to enable the user to run the code with larger time steps without losing accuracy. Implementing a non-uniform grid in the code can also be beneficial in terms of both accuracy and rate of convergence. In laminar flame modeling, due to the high gradients of variables in the reacting layers, a large number of control volumes is 
required to maintain the solution accuracy. In other parts of the domain, a smaller number of the control volumes is sufficient in order to maintain the solution accuracy. Thus, in applications in which a transient boundary condition is applied, implementing the second order temporal discretization and adaptive gridding can be helpful in order to have faster convergence, even in higher frequencies.

2. In this thesis, the stoichiometric condition of a methane/air flame was compared to fuel lean cases due to their significance in the literature and applications in industry. A study on fuel rich cases of the same flame can be valuable in order to compare to the current results and those of similar analyses in the counter-flow geometry. A different fuel type such as propane or hydrogen for different Lewis Numbers are also suggested to be studied due to the importance of flame burning rate which leads to different behavior.

3. More physical insights can be investigated by using the proposed model and generating the flame structure in the important points on a transient plot of input versus output. From the flame structure point of view, this approach requires special cautions to accurately capture instants by producing detailed graphs of different points. 


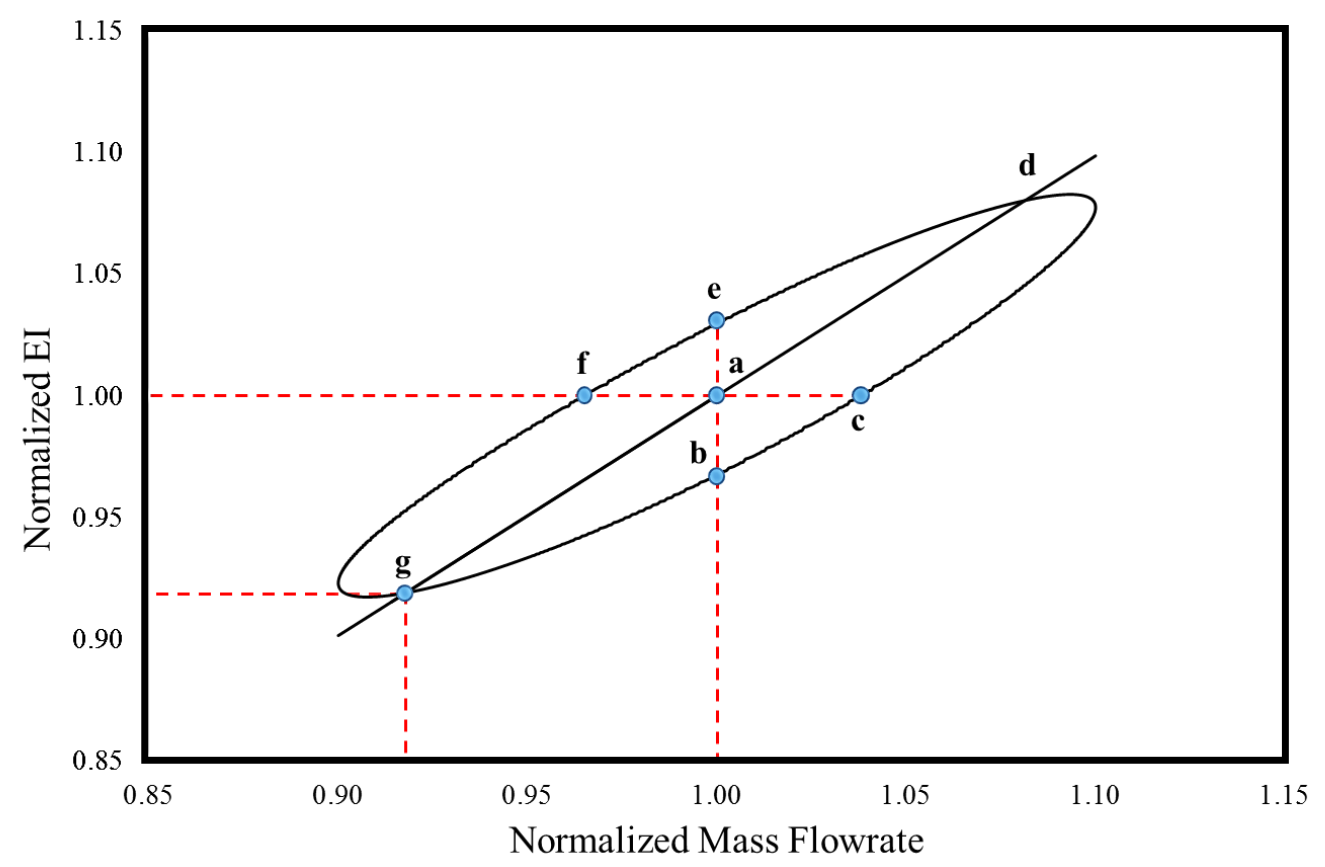

For instance, the flames corresponding to three points, e, a, and b have the same mass flow rate but different amounts of energy released in the domain. Thus, it can be concluded that the history of the flame, when exposed to a flow fluctuation, is important to capture the correct structure and properties such as heat release and burning rate. Another important phenomenon occurs at points $\mathrm{d}$ and $\mathrm{g}$, where the plot indicates different histories for each path (transient and steady responses). At this point, although the flame has the same mass flow rate and heat release, it passes through different paths with different histories which may result in different properties depending on the direction the flame moves (expanding or contracting). In other words, at point $\mathrm{g}$, the path for a quasi-steady response for both expanding and contracting flame is the same. However, for the transient flame, point g only shows the expanding path. 
4. The transient response of laminar flames has a very important application in flame extinction limits. Similar works in the counter flow geometry have shown that the quasi-steady response of a flame depicts a wider region for extinction limits. However, the transient response of a strained flame showed a smaller region due to the attenuated amplitude and delayed response of the flame. A potential variation in extinction limits of a curved flame can be valuable with regard to the previous works.

5. Using the complex chemistry instead of simple reduced reactions in simulating a flame leads to several time scales. Therefore, in order to find an appropriate filter for applying the transient effects to a flamelet library, the transient response of the flame can be expressed and studied in terms of various quantities. A premixed laminar flame structure is divided into two layers, the fuel cracking zone and the intermediate reaction zone. Each of these layers has a separate thickness and time scale and accordingly a different transient response. The maximum flame temperature $\left(T_{\max }\right)$ and integrated fuel consumption rate $\left(W_{f}\right)$ can be candidates due to their frequent use in flamelet libraries.

6. Since the effects of transient response of laminar premixed flames are stored in the transfer functions, it would be advantageous to apply this method to flamelet libraries in order to include the unsteady stretch rate effects on local burning rate when the flamelets are curved. The combination of transient response of a curved flame and strained flames can constitute the total stretch rate in turbulent combustion modeling.

7. Another useful insight from the developed model is to study the actual curvature effects on steady state flame burning rate. In other words, how small could the radius of curvature be before the flame stop behaving like a planar flame? 


\section{References}

[1] T. D. Dunstan, N. Swaminathan and K. N. C. Bray, "Influence of Flame Geometry on Turbulent Premixed Flame Propagation: A DNS Investigation," Journal of Fluid Mechanics, vol. 709, pp. 191-222, 2012.

[2] N. Peters, "Laminar Flamelet Concepts in Turbulent Combustion," Twenty-first Symposium (International) on Combustion/The Combustion Institute," vol. 21, no. 1, pp. 1231-1250, 1988.

[3] J. Warnatz, G. Stahl, "Numerical Investigation of Time-dependent Properties and Extinction of Strained Methane- and Propane-air Flamelets," Combustion and Flame, vol. 85, pp. 285-299, 1991.

[4] N. Darabiha, S. M. Candel, F. E. Marble, "The Effect of Strain Rate on a Premixed Laminar Flame," Combustion and Flame, vol. 64, pp. 203-217, 1986.

[5] L. W. Kostiuk and K. N. C., Bray, "Mean Effects of Stretch on Laminar Flamelets in a Premixed Turbulent Flame," Combustion Science and Technology, vol. 95, pp. 193-212, 1993.

[6] H. S. Preetham and T. Lieuwen, "Dynamics of Laminar Premixed Flames Forced by Harmonic Velocity Disturbances," Journal of Propulsion and Power, vol. 24, no. 6, pp. 1390-1402, 2008.

[7] C. A. Ghoniem, A. F. Petrov, "The Transient Response of Strained Laminar-Premixed Flames," Combustion and Flame, vol. 102, pp. 401-417, 1995.

[8] M. D. Smooke, "The Computation of Laminar Flames," Proceedings of the Combustion Institute, vol. 34, p. 65-98, 2013.

[9] J. O. Hirschfelder, C. F. Curtiss, and D. E. Campbell, "Theory of Flame Propagation," Journal of Physical Chemistry, vol. 57, pp. 403-414, 1953.

[10] D. Spalding, "The Theory of Flame Phenomena with a Chain Reaction," Philosophical Transactions of the Royal Society of London. Series A, Mathematical and Physical Sciences, vol. 249, pp. 1-25, 1956.

[11] S. Margolis, "Time-Dependent Solution of a Premixed Laminar Flame," Journal of Computational Physics, vol. 27, pp. 410-427, 1978.

[12] G. Dixon-Lewis, "Flame Structure and Flame Reaction Kinetics: V. Investigation of Reaction Mechanism in a Rich Hydrogen + Nitrogen + Oxygen Flame by Solution of Conservation Equations," Proceedings of the Royal Society of London A, vol. 317, pp. 235-263, 1970.

[13] N. A. Eaves, A. Veshkini, C. Riese, Q. Zhang, S. B. Dworkin, M. J. Thomson, "CoFlame: A Refined and Validated Numerical Algorithm for Modeling Sooting Laminar Coflow Diffusion Flames," Computer Physics Communications, vol. 207, p. 464-477, 2016.

[14] R. R. Dobbins and M. D. Smooke, "A Fully Implicit, Compact Finite Difference Method for the Numerical Solution of Unsteady Laminar Flames," Flow, Turbulence and Combustion, vol. 85, p. 763-799, 2010.

[15] S. B. Dworkin, B. C. Connelly, A. M. Schaffer, B. A. V. Bennett, M. B. Long, M. D. Smooke, M. P. Puccio, B. McAndrews, J. H. Miller, "Computational and Experimental 
Study of a Forced, Time-dependent, Methane-air Coflow Diffusion Flame," Proceedings of the Combustion Institute, vol. 31, p. 971-978, 2007.

[16] N. A. Eaves, A. Veshkini, C. Riese, Q. Zhang, S. B. Dworkin, M. J. Thomson, "A Numerical Study of High Pressure, Laminar, Sooting, Ethane-air Coflow Diffusion Flames," Combustion and Flame, vol. 159, no. 10, pp. 3179-3190, 2012.

[17] M. D. Smooke, "Error Estimate for the Modified Newton Method with Applications to the Solution of Nonlinear, Two-Point Boundary Value Problems," Journal of Optimization Theory and Applications, vol. 39, no. 4, pp. 489-511, 1983.

[18] M. D. Smooke, R. E. Mitchell, and D. E. Keyes, "Solution of Premixed and Counterflow Diffusion Flame Problems by Adaptive Boundary Value Methods," in Numerical Boundary Value ODEs, Basel, Birkhauser, pp. 303-317, 1985.

[19] H. Pitsch, M. Chen, and N. Peters, "Unsteady Flamelet Modeling of Turbulent Hydrogen-air Diffusion Flames," Proceedings of the Combustion Institute, vol. 27, pp. 1057-1064, 1998.

[20] S. Taing, A. R. Masri, S. B. Pope, "PDF Calculations of Turbulent Nonpremixed Flames of $\mathrm{H}_{2} / \mathrm{CO}_{2}$ using Reduced Chemical Mechanisms," Combustion and Flame, vol. 95, pp. 133-150, 1993.

[21] M. Muradoglu, K. Liu, S. B. Pope, "PDF Modeling of a Bluff- Body Stabilized Turbulent Flame," Combustion and Flame, vol. 132, pp. 115-137, 2003.

[22] E. R. Hawkes and J. H. Chen, "Direct Numerical Simulation of Hydrogen-Enriched Lean Premixed Methane-Air Flames," Combustion and Flame, vol. 138, pp. 242-258, 2004.

[23] H. Pitsch, O. Desjardins, G. Balarac and M. Dime, "Large-Eddy Simulation of Turbulent Reacting Flows," Progress in Aerospace Sciences, vol. 44, pp. 466-478, 2008.

[24] Y. Xu, M. D. Smooke, "Application of a Primitive Variable Newton's Method for the Calculation of an Axisymmetric Laminar Diffusion Flame," Journal of Computational Physics, vol. 104, no. 1, p. 99-109, 1993.

[25] S. Tang, M. K. Chernovsky, H. G. Im, A. Atreya, "A Computational Study of Spherical Diffusion Flames in Microgravity with Gas Radiation Part I: Model Development and Validation," Combustion and Flame, vol. 157, no. 1, p. 118-126, 2010.

[26] S. W. Armfield, "Finite Difference Solutions of the Navier-Stokes Equations on Staggered and Non-Staggered Grids," Computers Fluids, vol. 20, no. 1, pp. 1-17, 1991.

[27] B. Karlovitz, D. Denniston, D. Knapschafer, F. Wells, "Studies on Turbulent flames 1: A. Flame Propagation Across velocity gradients B. Turbulence Measurement in Flames," Proceedings of the Combustion Institute, vol. 4, no. 1, p. 613-620, 1953.

[28] G. Markstein, Nonsteady Flame Propagation, New York: McMillan Publication, 1964.

[29] M. Matalon, "On Flame Stretch," Combustion Science and Technology, vol. 31, no. 3-4, pp. 169-181, 1983.

[30] S. M. Candel, T. J. Poinsot, "Flame Stretch and the Balance Equation for the Flame Area," Combustion Science and Technology, vol. 70, no. 1-3, pp. 1-15, 1990.

[31] M. L. Frankel, G. I. Sivashinsky, "On Effects due to Thermal Expansion and Lewis Number in Spherical Flame Propagation," Combustion Science and Technology, vol. 31, no. 3-4, pp. 131-138, 1983. 
[32] R. S. Cant, K. N. C. Bray, L. W. Kostiuk, B. Rogg, "Flow Divergence Effects in Strained Laminar Flamelets for Premixed Turbulent Combustion," Combustion Science and Technology, vol. 95, no. 1-6, pp. 261-276, 1993.

[33] T. Poinsot, D. Veynante, Theoretical and Numerical Combustion, Philadelphia: Edwards Inc., 2005.

[34] T. Saitoh, Y. Otsuka, "Unsteady Behaviour of Diffusion Flames and Premixed Flames for Counter Flow Geometry," Combustion Science Technology, vol. 12, no. 4, pp. 135-146, 1976.

[35] A. F. Ghoniem, C. A. Petrov, "The Transient Response of Strained Laminar-Premixed Flames," Combustion and Flame, vol. 102, pp. 401-417, 1995.

[36] F. Zhang, T. Zirwes, P. Habisreuther, H. Bockhorn, "Effect of Unsteady Stretching on the Flame Local Dynamics," Combustion and Flame, vol. 175, p. 170-179, 2017.

[37] R. Lauvergne, F. N. Egolfopoulos, "Unsteady Response of C3H8/AIR Laminar Premixed Flames Submitted to Mixture Composition Oscillations," Proceedings of the Combustion Institute, vol. 28, p. 1841-1850, 2000.

[38] Z. Huang, J. K. Bechtold, M. Matalon, "Weakly Stretched Premixed Flames in Oscillating Flows," Combustion Theory and Modelling, vol. 2, p. 115-133, 1998.

[39] G. K. Giannakopoulos, A. Gatzoulis, C. E. Frouzakis, M. Matalon, A. G. Tomboulides, "Consistent Definitions of "Flame Displacement Speed" and "Markstein Length" for Premixed Flame Propagation," Combustion and Flame, vol. 162, no. 4, p. 1249-1264, 2015.

[40] J. Qian, J. K. Bechtold, and C. K. Law, "On the Response of Spherical Premixed Flames under Rotation," Combustion and Flame, vol. 110, pp. 78-91, 1997.

[41] K. T. Aung, M. I. Hassan, S. Kwon, L. K. Tseng, O. C. Kwon, and G. M. Faeth, "Flame/Stretch Interactions in Laminar and Turbulent Premixed Flames," Combustion Science and Technology, vol. 174, pp. 61-99, 2002.

[42] H. Berestycki, G. I. Sivashinsky, "Flame Extinction by a Periodic Flow Field," SIAM, Journal on Applied Mathematics, vol. 51, pp. 344-350, 1991.

[43] R. C. Aldredge, "The Propagation of Wrinkled Premixed Flames in Spatially Periodic Shear Flow," Combustion and Flame, vol. 90, pp. 121-133, 1992.

[44] R. C. Aldredge, "The Speed of Isothermal-Front Propagation in Isotropic, Weakly Turbulent Flows," Combustion Science and Technology, vol. 178, p. 1201-1215, 2006.

[45] G. Nivarti, S. Cant, "Direct Numerical Simulation of the Bending Effect in Turbulent Premixed Flames," Proceedings of the Combustion Institute, vol. 36, no. 2, p. 19031910, 2017.

[46] R. C. Aldredge, "Flame-Surface Advection in Transient Periodic Flow," Combustion Science and Technology, vol. 187, p. 148-161, 2015.

[47] R. Aldredge, "Flame Propagation in Multiscale Transient Periodic Flow," Combustion and Flame, vol. 183, p. 166-180, 2017.

[48] T. Poinsot, D. Veynante, Theoretical and Numerical Combustion, Philadelphia: Edwards Inc., 2005. 
[49] T. Schuller, S. Ducruix, D. Durox, S. Candel, "Modeling Tools for the Prediction of Premixed Flame Transfer Functions," Proceedings of the Combustion Institute, vol. 29, p. 107-113, 2002.

[50] H. Y. Wang, C. K. Law, T. Lieuwen, "Linear response of stretch-affected premixed flames to flow oscillations," Combustion and Flame, vol. 156, p. 889-895, 2009.

[51] P. Palies, D. Durox, T. Schuller, S. Candel, "Nonlinear Combustion Instability Analysis Based on the Flame Describing Function Applied to Turbulent Premixed Swirling Flames," Combustion and Flame, vol. 158, p. 1980-1991, 2011.

[52] T. Schuller, D. Durox, S. Candel, "A Unified Model for the Prediction of Laminar Flame Transfer Functions: Comparisons Between Conical and V-Flame Dynamics," Combustion and Flame, vol. 134, p. 21-34, 2003.

[53] R. Balachandran, B. O. Ayoola, C. F. Kaminski, A. P. Dowling, E. Mastorakos, "Experimental Investigation of the Nonlinear Response of Turbulent Premixed Flames to Imposed Inlet Velocity Oscillations," Combustion and Flame, vol. 143, p. 37-55, 2005.

[54] T. Lieuwen, "Nonlinear Kinematic Response of Premixed Flames to Harmonic Velocity Disturbances," Proceedings of the Combustion Institute, vol. 30 , p. 1725-1732, 2005.

[55] S. Ducruix, D. Durox, S. Candel, "Theoretical and Experimental Determinations of the Transfer Function of a Laminar Premixed Flame," Proceedings of the Combustion Institute, vol. 28, p. 765-773, 2000.

[56] H. G. Im, L. L. Raja, R. J. Kee, Linda R. Petzold, "A Numerical Study of Transient Ignition in a Counterflow Nonpremixed Methane-Air Flame using Adaptive Time Integration," Combustion Science and Technology, vol. 158, no. 1, pp. 341-363, 2000.

[57] S. D. Tse, D. Zhu, C. J. Sung, Y. Ju, C. K. Law, "Microgravity Burner-Generated Spherical Diffusion Flames: Experiment and Computation," Combustion and Flame, vol. 125, p. 1265-1278, 2001.

[58] G. Billet, V. Giovangigli \& G. de Gassowski, "Impact of Volume Viscosity on a Shockhydrogen-bubble Interaction," Combustion Theory and Modelling, vol. 12, no. 2, pp. 221-248, 2008.

[59] O. J. Teerling, A. C. Mcintosh, J. Brindley, "Pressure Wave Excitation of Natural Flame Frequencies," Combustion Theory and Modelling, vol. 11, no. 1, p. 147-164, 2007.

[60] G. Emanuel, "Effect of Bulk Viscosity on a Hypersonic Boundary Layer," Physics of Fluids A, vol. 4, p. 491-495, 1992.

[61] B. Muller, "Low Mach Number Asymptotics of the Navier-Stokes Equations and Numerical Implications," in 30th Computational Fluid Dynamics Lecture Series, von Karman Institute for Fluid Dynamics, 8-12 March, 1999.

[62] G. M. Faeth, J. P. Gore, S. G. Chuech, and S-M. Jeng, in Annual Reviews of Numerical Fluid Mechanics, Hemisphere, New York, 1989, 1-38.

[63] Y. R. Jun Ji, Sivathanu and J. P. Gore, "Thermal Radiation Properties of Turbulent Lean Premixed Methane Air Flames," Proceedings of the Combustion Institute, vol. 28, p. 391-398, 2000. 
[64] R. J. Kee, J. A. Miller, T. H. Jefferson, "Chemkin: A General-purpose, Problem-independent, Transportable Fortran Chemical Kinetics Code Package, Technical Report SAND808003," Sandia National Laboratories, 1980.

[65] R. J. Kee, J. Warnatz, J. A. Miller, "A Fortran Computer Code Package for the Evaluation of Gasphase Viscosities, Conductivities, and Diffusion Coefficients Technical Report SAND83-8209," Sandia National Laboratories, 1983.

[66] G. D. Thiarl, "Improved Finite-Difference Scheme for the Solution of Convection-Diffusion Problems with the SIMPLEN Algorithm," Numerical Heat Transfer, vol. 18, pp. 81-95, 1990.

[67] S. V. Patankar, Numerical Heat Transfer and Fluid Flow, New York, 1980.

[68] S. B. Dworkin, J. A. Cooke, B. A. V. Bennett, B. C. Connelly, M. B. Long, M. D. Smooke, R. J. Hall and M. B. Colket, "Distributed-Memory Parallel Computation of a Forced, Time-Dependent, Sooting, Ethylene/Air Coflow Diffusion Flame," Combustion Theory and Modelling, vol. 13, no. 5, p. 795-822, 2009.

[69] M. D. Smooke, "Solution of Burner-Stabilized Premixed Laminar Flames by Boundary Value Methods," Journal of Computational Physics, vol. 48, pp. 72-105, 1982.

[70] H. A. Van Der Vorst, "Bi-CGSTAB: A Fast and Smoothly Converging Variant of Bi-CG for the Solution of Nonsymmetric Linear Systems," SIAM Journal on Scientific and Statistical Computing, vol. 13, p. 631-644, 1992.

[71] S. B. Dworkin, B. A. V. Bennett, M. D. Smooke, "A Mass-Conserving Vorticity-Velocity Formulation with Application to Nonreacting and Reacting Flows," Journal of Computational Physics, vol. 215, p. 430-447, 2006.

[72] M. Saad, M. H. Schultz, "GMRES: A Generalized Minimal Residual Algorithm for Solving Nonsymmetric Linear Systems," SIAM Journal on Scientific and Statistical Computing, vol. 7, pp. 856-869, 1986.

[73] T. Poinsot, S. Candel, and A. Trouvé, "Applications of Direct Numerical Simulation to Premixed Turbulent Combustion," Progress in Energy and Combustion Science, vol. 6, no. 21, pp. 531-576, 1995.

[74] G. P. Smith, D. M. Golden, M. Frenklach, N. W. Moriarty, B. Eiteneer, M. Goldenberg, C. T. Bowman, R. K. Hanson, S. Song, W. C. Gardiner, Jr., V. V. Lissianski, and Z. Qin, "http://www.me.berkeley.edu/gri_mec," [Online].

[75] I. B. Celik, U. Ghia, P. J. Roache and C. J. Freitas, "Procedure of Estimation and Reporting of Uncertainty Due to Discretization in CFD Applications," Journal of Fluids Engineering, vol. 130, no. 7, 2008.

[76] D. Dandy, "http://navier.engr.colostate.edu/ dandy/code/code-4/index.html," 2015. [Online].

[77] M. W. Chase Jr., C. A. Davies, J. R. Davies, Jr., D. J. Fulrip, R. A. McDonald, and A. N. Syverud, JANAF Thermochemical Tables, Journal of Physical and Chemical Reference Data, 1985.

[78] C. M. Vageiopouios, F. N. Egolfopouios, "Direct Experimental Determination of Laminar Flame Speeds," Twenty-Seventh Symposium (International) on Combustion/The Combustion Institute, vol. 27, no. 1, p. 513-519, 1998. 
[79] E. Hu, X. Li, X. Meng, Y. Chen, Y. Cheng, Y. Xie, Z. Huang, "Laminar Flame Speeds and Ignition Delay Times of Methane-Air Mixtures at Elevated Temperatures and Pressures," Fuel, vol. 158, p. 1-10, 2015.

[80] I. G. Shepherd, J. B. Moss, "Characteristic Scales for Density Fluctuations in a Turbulent Premixed Flame," Combustion Science and Technology, vol. 33, no. 5-6, pp. 231-243, 1983.

[81] J. Driscoll, "Turbulent Premixed Combustion: Flamelet Structure and its Effect on Turbulent Burning Velocities," Progress in Energy and Combustion Science, vol. 34, no. 1, pp. 91134, 2008.

[82] G. K. Giannakopoulos, M. Matalon, C. E. Frouzakis, A. G. Tomboulides, "The Curvature Markstein Length and the Definition of Flame Displacement Speed for Stationary Spherical Flames," Proceedings of the combustion institute, vol. 35, p. 737-743, 2015.

[83] M. Sahafzadeh, L. W. Kostiuk, S. B. Dworkin, "Transient Response of a Laminar Premixed Flame to a Radially Diverging/Converging Flow," Combustion and Flame, vol. 179, p. 51-62, 2017.

[84] R. Vidal, "Recursive Identification of Switched ARX Systems," Automatica, vol. 44, no. 9, pp. 2274-2287, 2008.

[85] N. Noiray, D. Durox, T. Schuller And S. Candel, "A Unified Framework for Nonlinear Combustion Instability Analysis Based on the Flame Describing Function," Journal of Fluid Mechanics , vol. 615, p. 139-167, 2008.

[86] A. P. Dowling, "Nonlinear Self-Excited Oscillations of a Ducted Flame," Journal of Fluid Mechanics, vol. 346, p. 271-290, 1997.

[87] M. Schetzen, The Volterra and Wiener Theories of Nonlinear Systems, Krieger Publishing Company, 2006.

[88] A. Wills, T. B. Schön, Lennart Ljung, Brett Ninness, "Identification of Hammerstein-Wiener Models," Automatica, vol. 49, no. 1, pp. 70-81, 2013.

[89] A. Rahrooh, S. Shepard, "Identification of Nonlinear Systems Using NARMAX Model," Nonlinear Analysis: Theory, Methods \& Applications, vol. 71, no. 12, pp. e1198-e1202, 2009.

[90] T. L. Santosh Hemchandra, "Local Consumption Speed of Turbulent Premixed Flames - An Analysis of "Memory Effects"," Combustion and Flame, vol. 157, no. 5, p. 955-965, 2010.

[91] Q. Zhang, "Using Wavelet Network in Nonparametric Estimation," IEEE Transactions on Neural Networks and Learning Systems, vol. 8, p. 227-236, 1997. 